\title{
NANOPARTICLES AND POLYMER CRYSTALLIZATION KINETICS IN HYBRID ELECTRONIC DEVICES
}

\author{
A Thesis \\ presented to \\ the Faculty of California State Polytechnic State University, \\ San Luis Obispo
}

\author{
In Partial Fulfillment \\ of the Requirements for the Degree \\ Master of Science in Polymers and Coatings Science
}

by Taylor William Wagner

December 2013 
(C) 2013

Taylor William Wagner

ALL RIGHTS RESERVED 


\section{COMMITTEE MEMBERSHIP}

TITLE:

AUTHOR:

DATE SUBMITTED:

COMMITTEE CHAIR:

COMMITTEE MEMBER:

COMMITTEE MEMBER:
Nanoparticles and Polymer Crystallization Kinetics in Hybrid Electronic Devices

Taylor William Wagner

December 2013

Shanju Zhang, PhD

Assistant Professor of Chemistry

Derek Gragson, PhD

Professor of Chemistry

Chad Immoos, PhD

Associate Professor of Chemistry 


\section{ABSTRACT \\ Nanoparticles and Polymer Crystallization Kinetics in Hybrid Electronic Devices}

\section{Taylor William Wagner}

Conjugated semi-conducting polymers have become well known for their potential applications in hybrid electronic devices like solar cells, LEDs, and organic displays. These hybrid devices also contain inorganic nanoparticles, which complement the polymer when they are combined into the same layer. Control over the conformation and crystallinity of the polymer is critical for device performance, yet not much is known about the effect that these nanoparticles have on the polymer. Here, zinc oxide nanowire was surface modified with mono-substituted-carboxylic acid tetraphenylporphyrin and dodecanethiol, and introduced to poly(3-hexyl thiophene) in solution. The electron transfer, kinetics, and thermodynamics of this system were investigated through spectroscopic methods. Chemical reaction rate laws and Lauritzen-Hoffman Growth Theory were employed to substantiate the mechanism and rate of polymer crystallization. Surface-modification of the $\mathrm{ZnO}$ nanowire suggested an improvement in polymer nucleation by as much as $43.8 \%$. A synthetic procedure was also developed to modify the inorganic nanowire with quantum dots in order to improve electron transport into the nanowire. Development of these theories and exploration of these surface effects can help lead the way for a new generation of flexible, high efficiency, hybrid electronic devices. 


\section{ACKNOWLEDGMENTS}

I would like to thank Dr. Shanju Zhang for giving me the chance to work on this amazing project and being such an incredibly active advisor. Dr. Zhang has always been extremely invested in his students' progress and has provided the graduate-level

academic environment needed to excel. Dr. Zhang has given me the ability to understand things on a deeper level and has given me confidence both in and out of the lab, and for this I am deeply appreciative.

I would also like to thank Neil Redeker, Grace Luo and the other members of the Zhang research group, who have all individually helped me understand parts of my project better than I could have myself. Their help and insight has been invaluable, and have made this research a fantastic experience.

I would like to express my appreciation to Dr. Ray Fernando and the Polymers and Coatings Program Faculty. If it was not for their advice and encouragement, none of this research would have happened.

I would also like to give a special thanks to Dr. John Hagen, Dr. Derek Gragson, Dr. Chad Immoos, Dr. Robert Echols, and Dr. David Braun, who have all devoted their time to help me understand parts of this research, or have given me the tools I needed to advance. I would also like to acknowledge Professor Richard Savage of the Materials Engineering Department and Dr. Junzuo Wang for graciously providing material.

Lastly, this project was made possible through funding from the Cal Poly Extramural Funding Initiative, National Science Foundation, and the William L. Frost Scholarship. 


\section{TABLE OF CONTENTS}

Page

List of Tables. . . . . . . . . . . . . . . . . . . . . . . . . . . . . .

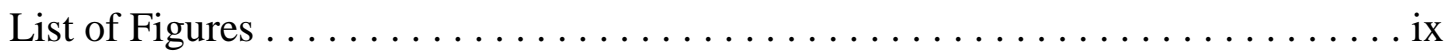
Chapter

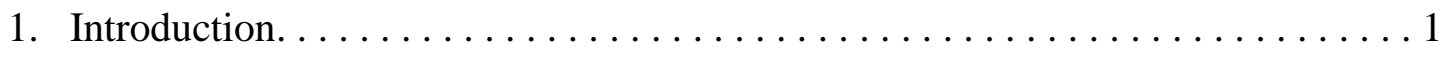

1.1. Organic Electronic Devices $\ldots \ldots \ldots \ldots \ldots \ldots \ldots \ldots \ldots \ldots \ldots$

1.2. Hybrid Devices and Device Architecture..................... 3

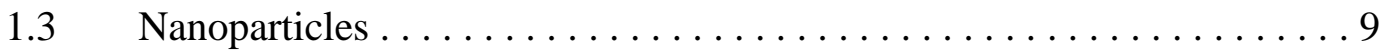

1.4 Conjugated Semi-conducting Polymers .................... 14

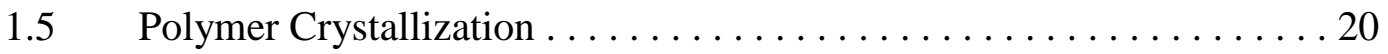

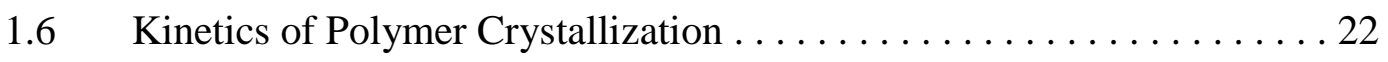

2. Experimental Methods .................................... 34

2.1. Material Preparation .............................. 34

2.1.1. Surface Modification of $\mathrm{ZnO}$ Nanowire............... 34

2.1.2. Quantum Dot Attachment to $\mathrm{ZnO}$ Nanowire .............. 35

2.2. Characterization and Analysis ........................ 36

2.2.1. Fourier Transform Infrared Spectroscopy ............... 36

2.2.2. UV-visible Spectroscopy...................... 37

2.2.3. Fluorescence Spectroscopy........................ 39

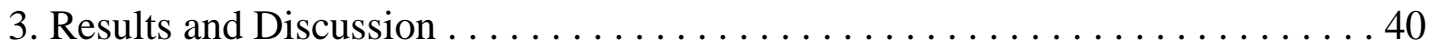

3.1. Interaction of Modified $\mathrm{ZnO}$ and P3HT $\ldots \ldots \ldots \ldots \ldots \ldots \ldots$

3.1.1. Characterization of Modified ZnO. .............. 40 
3.1.2. Effect of Modified Nanowire on P3HT Optical Properties . . . . 44

3.1.3. Kinetics of P3HT Crystallization ... . . . . . . . . . . . . 49

3.1.4. Avrami Analysis. . . . . . . . . . . . . . 57

3.1.5. Lauritzen-Hoffman Kinetics. . . . . . . . . . . . . . . . 59

3.2. Quantum Dot Modified Nanowire .................63

3.2.1 Quantum Dot Characterization ...............63

3.2.2 Effect of Quantum Dots on P3HT Fluorescence . . . . . . . . . . 64

3.2.3 Quantum Dot Attachment to Ligand. . . . . . . . . . . . . 65

3.3. Research Outlook ... . . . . . . . . . . . . . . . . . . . . . . . 68

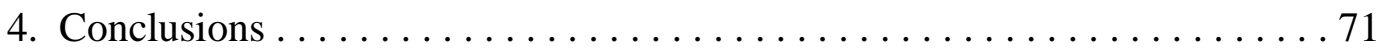

5. References. . . . . . . . . . . . . . . . . . . . . 72 


\section{LIST OF TABLES}

Table Page

3.1. Experimentally determined rate constants and reaction orders. . . . . . 55

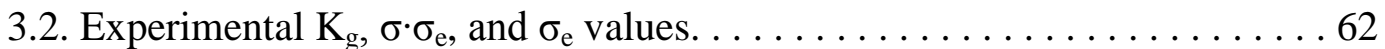




\section{LIST OF FIGURES}

Figure

Page

1.1. Diagram of (1) exciton generation, (2) diffusion of electron-hole pair to the interface, (3) dissociation, and $(4,5)$ charge transfer to electrodes in a two-layer organic solar cell. . . . . . . . . . . . . . . . 3

1.2. Record efficiencies of various research solar cells over time . . . . . . . 4

1.3. Diagram of a hybrid bilayer device, not to scale. Highlighted is a typical exciton diffusion length of $10 \mathrm{~nm} . \ldots \ldots \ldots \ldots \ldots \ldots \ldots \ldots \ldots \ldots \ldots \ldots \ldots \ldots$

1.4. Diagram of a bulk heterojunction device, not to scale. Highlighted is a typical exciton diffusion length of $10 \mathrm{~nm} . \ldots \ldots \ldots \ldots \ldots \ldots$

1.5. Diagram of an ordered heterojunction device, not to scale. Highlighted is a typical exciton diffusion length of $10 \mathrm{~nm} \ldots \ldots \ldots \ldots \ldots$

1.6. CdSe nanoparticles with (a) $7 \times 7 \mathrm{~nm}$, (b) $7 \times 30 \mathrm{~nm}$, and (c) $7 \times 60 \mathrm{~nm}$ dimensions. (d) Displays the effect of aspect ratio on external quantum efficiency, similar to $\mathrm{UV}$-visible absorbance . . . . . . . . . . . . 10

1.7. Tunneling electron microscopic (TEM) and cartoon images of $\mathrm{TiO}_{2}$ nanorods surface modified with (a) pyridine, (b) a Cu-phthalocyanine-ether dye, and (c) Oligo-3HT-(Br)COOH, $\mathrm{n} \approx 27$. The tendency of the nanorods to aggregate is decreased with long conjugated ligands. . . . . . . . 13

1.8. TEM images of (a) $\mathrm{TiO}_{2}$ nanowires, (b) $\mathrm{CdS}$ coated $\mathrm{TiO}_{2}$ nanowires, and

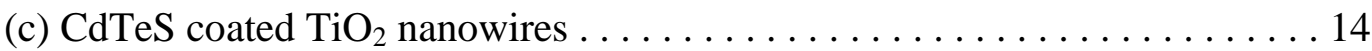


1.9. Chemical structures of common semiconducting CPs.

(a) poly(phenylenevinylene), (b) poly(phenylene), and (c) poly(thiophene). . 15

1.10. Diagram of HOMO and LUMO energy levels in polymer, where at infinite chain length a valence band and conduction band are formed. . . . . 16

1.11. Graphic depicting the effect of intramolecular chain interaction on

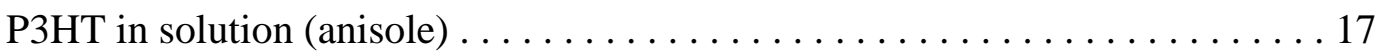

1.12. TEM image of crystallized $\mathrm{P} 3 \mathrm{HT}$ on a $\mathrm{CdS}$ nanowire surface $\ldots \ldots \ldots 18$

1.13. Direction of hopping transport and conduction transport in P3HT . . . . . 19

1.14. Types of models proposed for polymer crystallization under quiescent

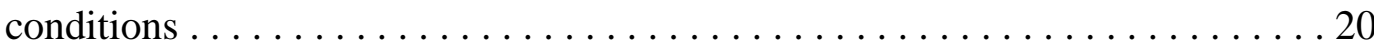

1.15. Scheme of the steps involved in the crystallization of P3HT . . . . . 22

1.16. Schematic of initial stem deposition in polymer crystallization. $A_{0}$ and $\mathrm{A}_{0}$ ' are the forward reaction rates of stem deposition, $\mathrm{B}_{1}$ and $\mathrm{B}_{1}$ ' are the reverse reaction rates of stem deposition, $\mathrm{l}_{\mathrm{g}}{ }^{*}$ is the average crystal stem length .25

1.17. Falling activation energy of subsequent polymer chain reentry . . . . . 26

1.18. Schematic of the different regimes involved in polymer crystallization. . . 28

1.19. Typical L-H plots used to determine the regime boundaries and $\mathrm{Kg}$

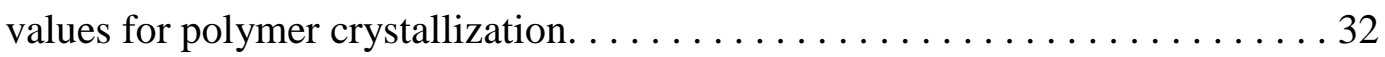

2.1. High-resolution TEM image of isolated pristine nanowires . . . . . . . . . 34 
2.2. Scheme of chemical structures used for modification of $\mathrm{ZnO}$ and $\mathrm{P} 3 \mathrm{HT}$ kinetics. (a) P3HT, (b) Modified $\mathrm{ZnO}$ with $\mathrm{R}$ representing one of two

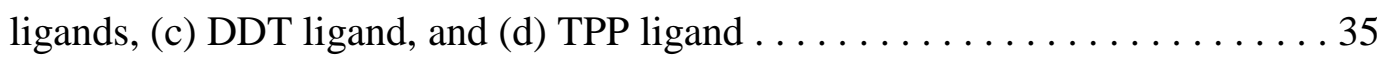

2.3. Reaction scheme and chemicals used in surface modification of $\mathrm{ZnO}$ nanowire with quantum dots. . . . . . . . . . . . . . 36

2.4. Inside of the Jasco UV-visible spectrometer used in this work with blank cuvette in the upper left and cuvette with P3HT solution at bottom . . . . . . 38

3.1. IR spectra of $\mathrm{ZnO}$, DDT, and DDT-modified $\mathrm{ZnO} \ldots \ldots \ldots \ldots \ldots$

3.2. IR spectra of TPP and TPP-modified $\mathrm{ZnO}$, with inset of zoomed in region from $1200-1800 \mathrm{~cm}^{-1}$ to highlight the disappearance of carbonyl stretching after modification $\ldots \ldots \ldots \ldots \ldots \ldots \ldots \ldots \ldots \ldots \ldots \ldots \ldots \ldots \ldots \ldots \ldots$

3.3. UV-visible spectra of $\mathrm{ZnO}$, DDT-modified $\mathrm{ZnO}$, TPP, and TPP-modified $\mathrm{ZnO}$. Inset is a zoomed in comparison of TPP and TPP-modified $\mathrm{ZnO}$ to highlight the shift in characteristic porphyrin peaks. . . . 44

3.4. Addition of modified $\mathrm{ZnO}$ showing a lack of effect on the fluorescence

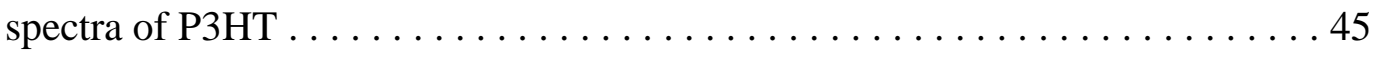

3.5. P3HT thermochromism displayed via UV-visible spectroscopy. (a) The temperature dependence of $\mathrm{P} 3 \mathrm{HT}$ conformation, (b) Heating and cooling curves of P3HT taken at a $\lambda$ of $600 \mathrm{~nm}$ in anisole $\ldots \ldots \ldots \ldots \ldots \ldots$ 
3.6. Cooling curves for P3HT, P3HT with DDT-modified ZnO, P3HT with TPP-modified ZnO, and P3HT with ZnO. Data reflects poor experimental procedure and displays the tendency for aggregate polymer formation

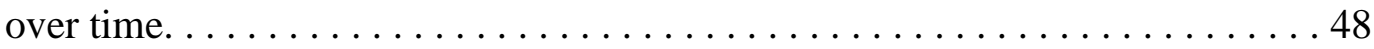

3.7. Correctly acquired cooling and heating curves of the four systems . . . . . 49

3.8. Kinetics of P3HT conformational change. (a) Isotherms showing the time dependence of P3HT crystallization, (b) Single sample P3HT crystallization kinetics, taken at a $\lambda$ of $600 \mathrm{~nm} \ldots \ldots \ldots \ldots \ldots \ldots$

3.9. Kinetics of P3HT crystallization at different crystallization temperatures $\mathrm{T}_{\mathrm{c}}$, measured by monitoring the $600 \mathrm{~nm}$ peak over time via UV-visible

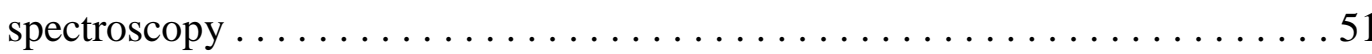

3.10. Concentration dependence of P3HT crystallization for (a) P3HT, (b) P3HT with $\mathrm{ZnO}$, (c) P3HT with DDT-modified $\mathrm{ZnO}$, and (d) P3HT with TPP-modified $\mathrm{ZnO}$. The kinetics of P3HT with $\mathrm{ZnO}$ in (b) were taken at a different $\mathrm{T}_{\mathrm{c}}$, leading to a drastically different appearance than the other three

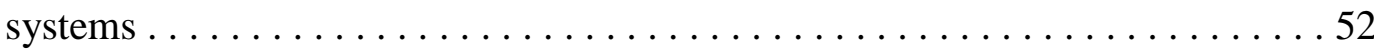

3.11. Experimental reaction rate data gathered via method of initial rates for P3HT, P3HT with ZnO, P3HT with DDT-modified ZnO, and P3HT with TPP-modified ZnO. All four systems are shown in (a), while the P3HT with $\mathrm{ZnO}$ kinetics (done at a different $\mathrm{T}_{\mathrm{c}}$ ) were removed in (b) for clarity . . . . . 53

3.12. Log-log plot of kinetic data for the determination of reaction order of P3HT, P3HT with ZnO, P3HT with DDT-modified $\mathrm{ZnO}$, and P3HT with TPP-modified ZnO. All four systems are shown in (a), while the P3HT with $\mathrm{ZnO}$ kinetics (done at a different $\mathrm{T}_{\mathrm{c}}$ ) were removed in $(\mathrm{b})$ for clarity . . . . . . 54 
3.13. Avrami analysis of P3HT crystallization via UV-visible spectroscopy displaying the temperature dependence of the Avrami constants k and $n$. . . . 57

3.14. Avrami analysis of P3HT, P3HT with $\mathrm{ZnO}, \mathrm{P} 3 \mathrm{HT}$ with DDT-ZnO and P3HT with TPP-ZnO on a short time scale $\ldots \ldots \ldots \ldots \ldots \ldots \ldots$

3.15. Determination of $\mathrm{T}_{\mathrm{d}}{ }^{0}$ for P3HT in anisole using the Organ and Keller method. The heating curves started at different $\mathrm{T}_{\mathrm{c}}$ 's shown in (a) were plotted against their estimated $\mathrm{T}_{\mathrm{d}}$ values in $(\mathrm{b}) \ldots \ldots \ldots \ldots \ldots \ldots \ldots \ldots \ldots \ldots \ldots \ldots \ldots \ldots$

3.16. Determination of $\mathrm{K}_{\mathrm{g}(1)}$ via L-H Theory for P3HT, P3HT with $\mathrm{ZnO}$, P3HT with DDT-modified ZnO, and P3HT with TPP-modified ZnO. . . . . . . 61

3.17. Characterization data for CdSe quantum dots. The temperature dependence of the UV-visible absorbance spectrum is explored in (a) and the concentration dependence on the fluorescence spectrum is displayed in (b) . . . 64

3.18. The effect of addition of quantum dots on the fluorescence spectrum of P3HT. No quenching is observed, and the spectra resemble the individual P3HT and quantum dot spectra, implying there is little to no interaction as

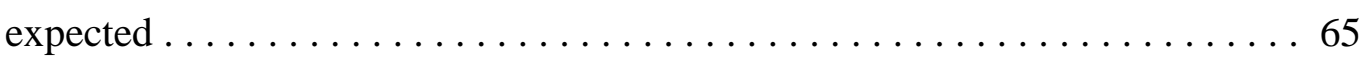

3.19. IR spectroscopy characterization data for CdSe quantum dots, APS ligand, and APS-modified $\mathrm{ZnO}$ attached to quantum dots. . . . . . . . . 66

3.20. IR spectroscopy characterization data for CdSe quantum dots, MPS ligand, and MPS-modified $\mathrm{ZnO}$ attached to quantum dots. . . . . . . . 66

3.21. Fluorescence micrographs of $\mathrm{ZnO}-\mathrm{CdSe}$ clusters on a $\mathrm{Si}$ wafer in air

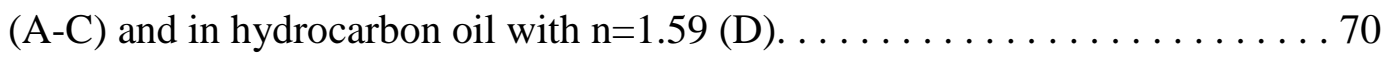




\section{Introduction}

\subsection{Organic Electronic Devices}

The first discovery of a conductive all-organic material can be attributed to Henry Letheby in $1862 .{ }^{[1]}$ It is believed Letheby produced polyaniline via anodic oxidation of aniline in sulfuric acid. This discovery did not yield any practical applications until the 1970’s when Shirakawa, Heeger, McDiarmid, began producing highly conductive films of polyacetylene. ${ }^{[2-6]}$ The idea of using these organic materials in electronic devices became more of a reality, and in 2000, these three scientists were recognized for their seminal achievements and were awarded the Nobel Prize in Chemistry "for the discovery and development of conductive polymers. ${ }^{,[7]}$ Researcher Ching W. Tang is also regarded as a key developer in the field for his construction of organic solar cells and light emitting diodes in $1981 .{ }^{[8]}$ Research on these materials in recent years has magnified, and many electronics retailers now offer organic displays for electronic devices as cost-competitive alternatives to traditional electronics.

Conductive organic polymers are already looking to replace traditional inorganic materials used in transistors, diodes, sensors, displays and photovoltaics. ${ }^{[9-10]}$ These semiconducting conjugated polymers (CPs) display similar electrical and optical properties of metals or semiconductors, yet they maintain more desirable mechanical and processing properties. Because of the plastic nature of these CPs, they can be incorporated into rollto-roll coating techniques and can produce flexible electronics and displays. ${ }^{[1]}$ They are also solution-processable, which provides a large economic benefit over traditional electronics and is largely the driving force behind the surge in organic devices. For these 
reasons, CPs have the potential to create a wide array of tailorable, lightweight, flexible, and low-cost organic devices. ${ }^{[9]}$

While CPs can be incorporated into a myriad of electronics, photovoltaic generation has been the research area of greatest interest due to the growing demand in clean energy resources. Here, the differences between organic and inorganic solar cells arise from the substantially different mechanisms of current generation. In typical inorganic devices for instance, a p-n junction is created by positively doping a semiconductor material and negatively doping a semiconductor material and placing them in contact. This creates an internal electric field that drives current generation when a photon strikes anywhere in the bulk of the materials to create a free charge carrier. ${ }^{[12]}$ In organic devices, instead of a p-n junction, a donor and acceptor are created. The donor material absorbs incoming photons and excites an electron from its Highest Occupied Molecular Orbital (HOMO) to its Lowest Unoccupied Molecular Orbital (LUMO) to create a photoexcitation pair called an exciton. ${ }^{[11]}$ The exciton is essentially a bound electron-hole pair, which must travel to a donor/acceptor interface to dissociate. Once the electron and hole have been dissociated, the electron travels to the cathode through the acceptor phase, and the hole travels to the anode through the donor phase, as seen in Figure 1.1. The electron eventually recombines through a back contact to create current. 


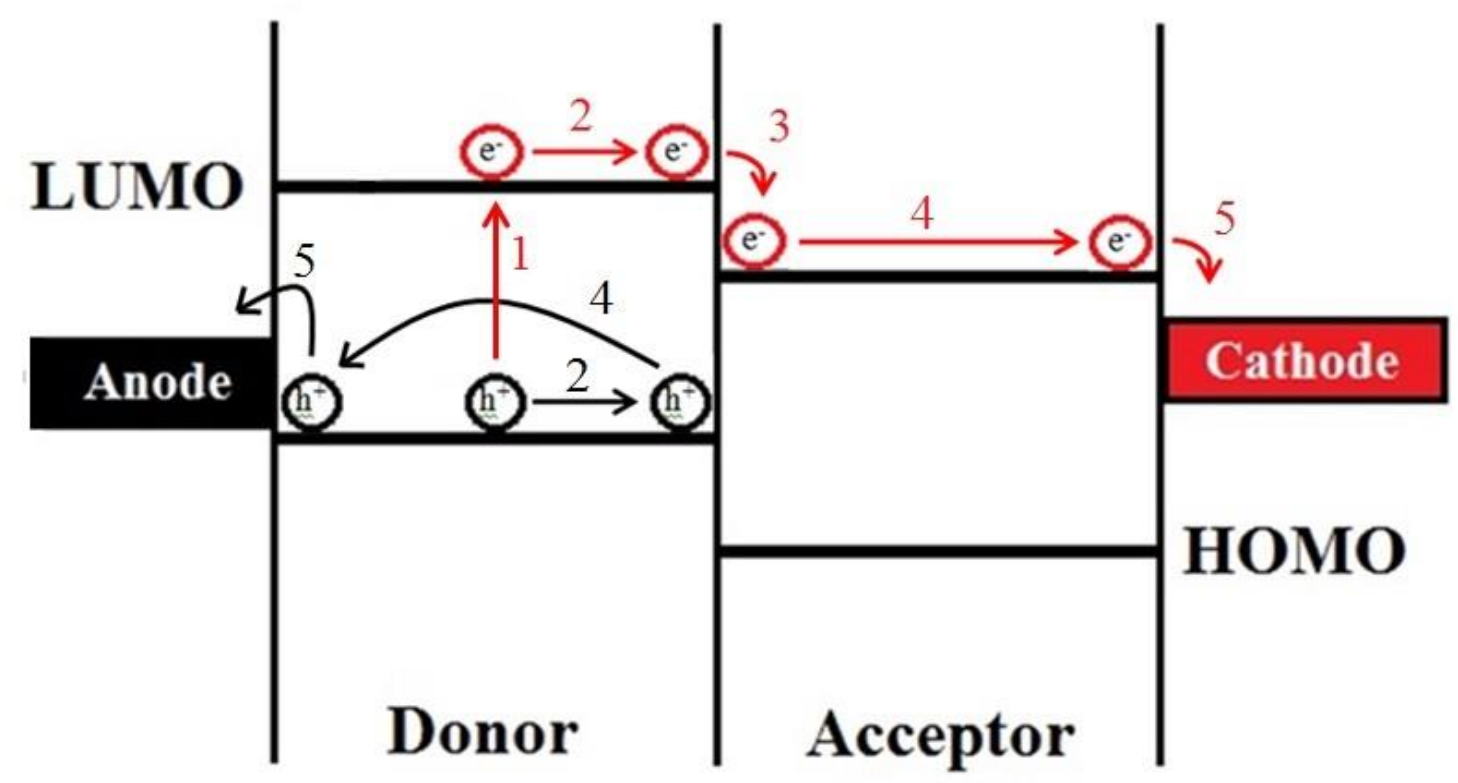

Figure 1.1. Diagram of (1) exciton generation, (2) diffusion of electron-hole pair to the interface, (3) dissociation, and (4,5) charge transfer to electrodes in a two-layer organic solar cell.

Although these devices boast many advantages, the technology is still relatively young and suffers from a few drawbacks. The theoretical limit of the power conversion efficiency is lower than that of inorganic solar cells, and is predicted to peak around 15\%. ${ }^{[11]}$ Organic photovoltaics are thus not expected to compete with inorganic solar cells in terms of efficiency, but can still become economically competitive because of fabrication simplicity and flexibility.

\subsection{Hybrid Devices and Device Architecture}

Organic electronics that incorporate CPs are still far from the efficiencies required to be commercially viable, yet the technology boasts so many advantages. Extensive research has focused upon discovering breakthrough technology that will allow the 
organic materials in these devices to achieve higher efficiencies and longer lifetimes. One solution to this problem is to create a hybrid device that combines both the organic and inorganic components in one device. The device is termed a hybrid because the active layer contains a combination of electron donor $\mathrm{CP}$ and electron acceptor inorganic nanostructures. This approach is fairly new, and because of this the best hybrid solar cells currently produce a very low power conversion efficiency (PCE) of around $3 \% .{ }^{[13]}$ Compared to records of about $24.7 \%$ PCE for single junction inorganic solar cells and above $10 \%$ for all-organic solar cells. ${ }^{[11,14]}$

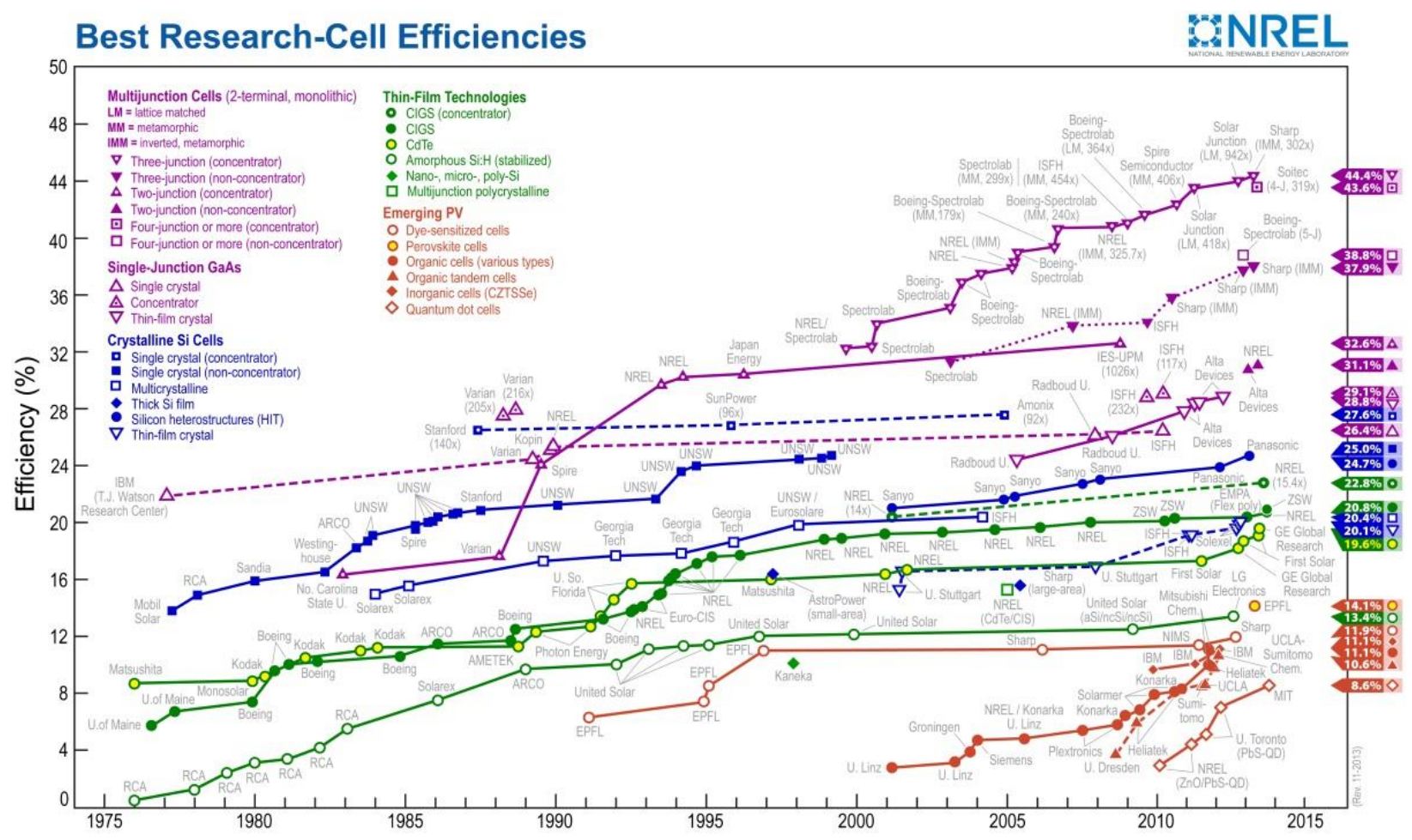

Figure 1.2. Record efficiencies of various research solar cells over time. ${ }^{[14]}$ 
Although hybrid solar cells seem to perform poorly, there is extensive motivation behind their continued exploration. Hybrid devices have the potential to combine the benefits of both organic and inorganic devices to produce a low-cost, highly efficient device. Unlike silicon wafers used in inorganic cells, nanoparticles used in hybrids are solution-processable and have a tunable band gap. This allows the entire hybrid to be solution-processable to maintain the flexibility aspect of organics without loss of current. These reasons make hybrid systems potentially the most cost-effective option for devices like solar cells, light-emitting diodes, and flexible displays of all available options. However, researchers are still struggling with the difficulty of incorporating two materials into one active layer and the interactions between the two in terms of charge transfer. One of the biggest problems in these hybrid devices is the proximity and interfacial area between the organic and inorganic phases. Several device architectures have been proposed to address this issue in order to maximize the interfacial area of the two phases to increase photocurrent generation.

The first device architecture is the most basic, and is referred to as a bilayer hybrid device, shown in Figure 1.3. Here, the donor layer lies below the acceptor layer, creating a small interfacial area between the two. These devices suffer from having a very small window of operation and thus produce little current. When incident photons strike the donor layer and excite an electron into the LUMO, the excited electron-hole pair has a small window of time to reach an interface, otherwise they recombine. This recombination will not create current, and thus any excitons that are created too far from an interface will not contribute to the overall current flow. The average distance an exciton can travel before recombining is termed the exciton diffusion length, and in 
polymers are typically on the order of $10 \mathrm{~nm}$. This is a fairly small distance in terms of the total device size, so large domain sizes tend to lower device performance. Because of this issue, other device architectures that increase the interfacial area are preferred over the simple hybrid bilayer device.

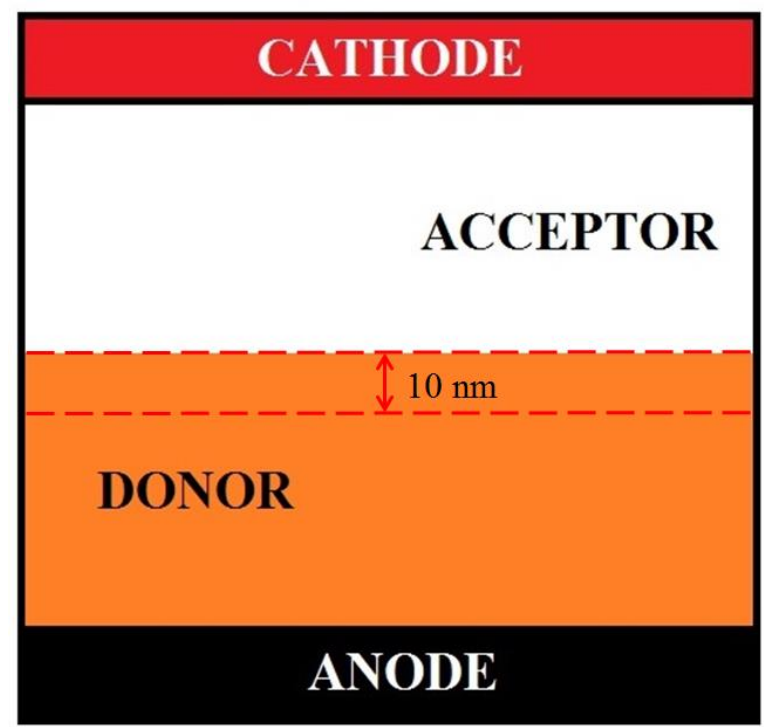

Figure. 1.3. Diagram of a hybrid bilayer device, not to scale. Highlighted is a typical exciton diffusion length of $10 \mathrm{~nm}$.

The bulk heterojunction (BHJ) device architecture, shown in Figure 1.4, incorporates both donor and acceptor phases into one layer. This creates a drastic increase in the interfacial area, but unfortunately contributes a few more concerns in charge transport. It is difficult to control the domain sizes of the materials in this layer, creating continuous areas of donor that are larger than $10 \mathrm{~nm}$ on all sides. This creates pockets of inactive material, which is made worse by the materials' tendency to phase separate during coating. A second issue arises from the tortuous pathways the electron and hole must travel to reach an electrode. The heterojunction layer will typically have areas of donor and acceptor that are discontinuous and do not connect directly to the electrodes. These "islands" result in the generation and trapping of charge carriers which cannot 
contribute to current generation. Here, the control of the morphology of the layers becomes crucial, and the miscibility and phase separation of the materials must be mitigated as much as possible. A substantial amount of research has gone into creating ligands that will bridge the gap between the organic donor and inorganic acceptor. ${ }^{[15-25]}$ These ligands are promising as they provide a simple cost-effective way to increase the effective interfacial area of the two materials, but they also introduce the issue of decreased charge transport and charge transfer. In general, devices that are fabricated with small or no ligands show higher PCE, but the fundamental effects of these ligands are still not well understood. Conjugated or polar ligands that can actively participate in exciton generation and transport are a potential solution, but have not been widely explored. $^{[13]}$

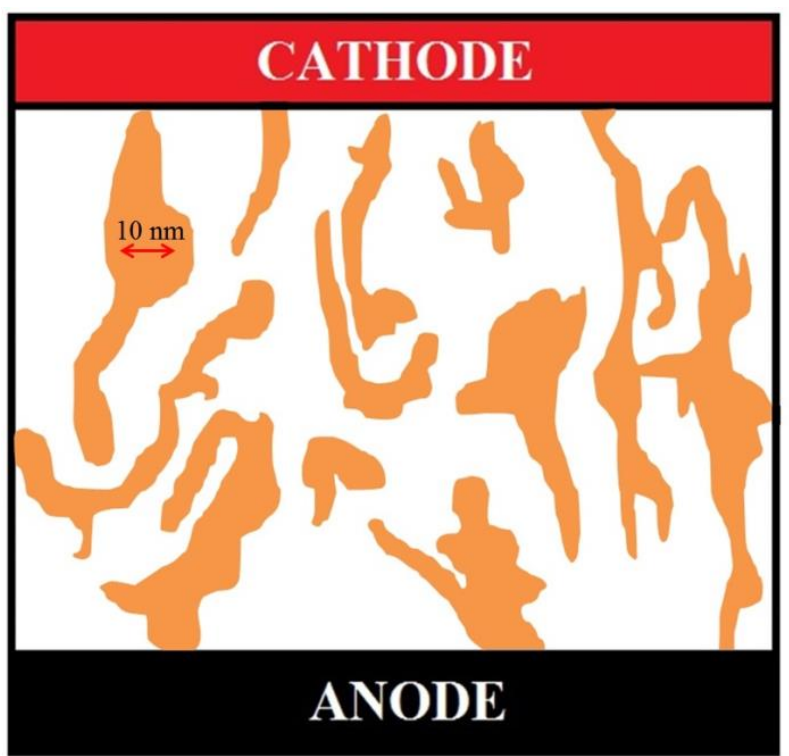

Figure 1.4. Diagram of a bulk heterojunction device, not to scale. Highlighted is a typical exciton diffusion length of $10 \mathrm{~nm}$.

The ideal device architecture that offers the highest amount of control is the ordered heterojunction, seen in Figure 1.5 In this architecture, the domains are fabricated to be precise widths of less than $10 \mathrm{~nm}$ and packed in a regular dense array. This 
arrangement provides the most benefits, but is understandably difficult to fabricate.

Several different approaches have been outlined in the literature attempting to achieve this morphology including di-block copolymers and nanoimprint lithography. ${ }^{[26]}$ This work will however detail improvements in the BHJ style architecture in order to improve donor and acceptor interactions. Ideally, future research will reveal a reliable method to create an ordered heterojunction and current BHJ systems can be directly adapted to the new architecture to produce a highly efficient device.

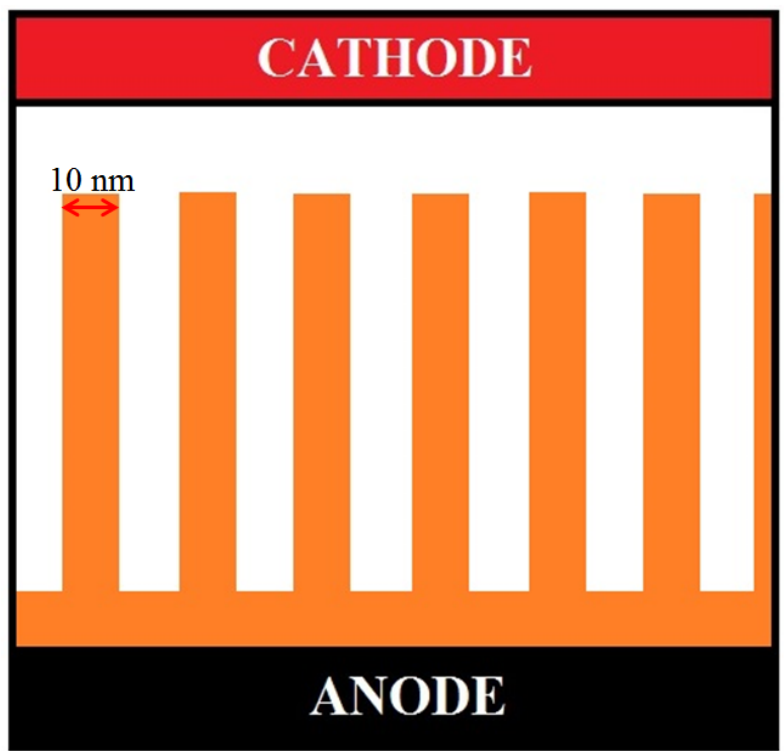

Figure 1.5. Diagram of an ordered heterojunction device, not to scale. Highlighted is a typical exciton diffusion length of $10 \mathrm{~nm}$. 


\subsection{Nanoparticles}

A nanoparticle is defined as a particle with one or more of its dimensions being under $100 \mathrm{~nm}$ in diameter. As stated previously, nanoparticles typically make up the inorganic component of hybrid electronics. This is because their small dimensions allow light to pass through to the organic layer and they provide high electrical conductivity. The high dielectric constant of these nanoparticles also decreases the coulombic attraction between electrons and holes, aiding in charge separation. Lastly, nanoparticles benefit from tunability of shape and size, which allows for more control of charge transport and band gap to line up with the polymer donor band gap. ${ }^{[27]}$

Nanoparticles are typically synthesized from multiple metals, such as GaAs, CdTe, $\mathrm{CdS}, \mathrm{CuInS}_{2}, \mathrm{PbSe}, \mathrm{PbS}, \mathrm{TiO}_{2}$, and ZnO. ${ }^{[13,28]}$ Nanoparticles can also be synthesized in a large variety of shapes and sizes, which have all seen uses in different applications. The most common nanoparticle structures seen in electronic applications are nano- dots, rods, tetrapods, wires and highly branched structures. ${ }^{[29]}$ These nanostructures can be divided into two categories: isotropic (quantum dots (QD)) and anisotropic (other nanoparticles). Nanoparticles are well known to exhibit exciton generation just like CPs with their band gap energy given by Equation 1.1. ${ }^{[29]}$

$$
\Delta E=\frac{h^{2} \pi^{2}}{2 R^{2}}\left(\frac{1}{m_{c}}+\frac{1}{m_{h}}\right)-\frac{1.768 e^{2}}{\varepsilon R}-0.248 E_{R_{y}}^{*}
$$

Although these particles can contribute largely to exciton generation in certain devices, the systems studied in this work incorporate around 10\% nanoparticle loading, and thus this principle will be largely ignored. Instead, the charge carrier mobility will be of greater focus, and more specifically the directionality of electron transport. The mechanism of electron transport in anisotropic nanoparticles is largely determined by 
their aspect ratio. Spherical nanodots must be in intimate contact with each other and produce current through electron hopping. Nanoparticles with large aspect ratios, called nanorods or nanowire, provide a continuous path for electron transport, and can be aligned vertically between electrodes to minimize tortuous pathways. ${ }^{\text {[27, 29-30] }}$
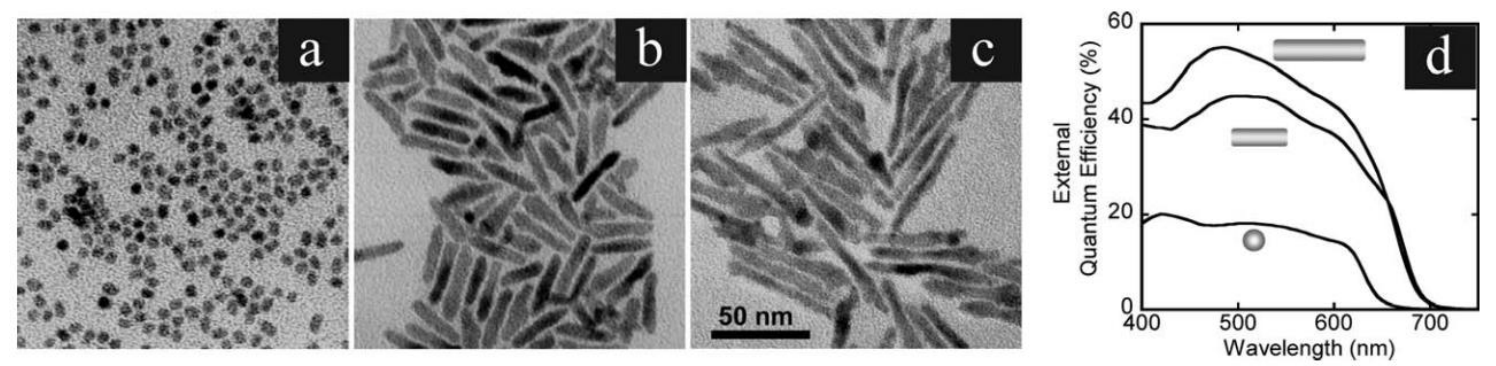

Figure 1.6. CdSe nanoparticles with (a) 7x7 nm, (b) 7x30 nm, and (c) 7x60 nm dimensions. (d) Displays the effect of aspect ratio on external quantum efficiency, similar to UV-visible absorbance. ${ }^{[30]}$

Nanotetrapods are yet another type of nanoparticle which has shown improvement in device efficiency when incorporated into a BHJ. The three-dimensional shape of the structures allows for more effective charge transport across the BHJ layer, but ultimately the larger size of the particle leads to short-circuiting and decreased solubility with CPs. $^{[13]}$

Isotropic nanostructures, like quantum dots, offer more advantages than other nanoparticles, but also come with a variety of additional challenges. Quantum dots are easily tunable, and thus their absorption can be tuned to cover a broad range of the UVvisible spectrum to compliment polymer absorption. They also boast high electron mobility and good photo- and chemical stability. Quantum dots also have strong electron injection properties to nearby materials. Previous experiments have even decorated CdS and $\mathrm{CdTeS}$ quantum dots onto $\mathrm{TiO}_{2}$ nanowires and have shown improved device efficiencies upwards of $350 \%$ compared to bare $\mathrm{TiO}_{2}$ nanowires. ${ }^{[31]}$ However, 
incorporating these particles into hybrid devices creates a challenge in developing a network where charges can transport directly to the electrode. Also, quantum dots tend to phase separate from the nonpolar CPs, decreasing interfacial area. This effect can only be partially mitigated by selection of solvent and processing techniques. ${ }^{[28]}$ Due to these limitations of both isotropic and anisotropic nanoparticles, strategies have emerged that attempt to mitigate the phase separation of organic and inorganic material and improve miscibility.

Because the organic polymer is typically a very long hydrophobic macromolecule and the inorganic nanoparticle surface is typically hydrophilic, many solutions involve modifying the outside of either the polymer or nanoparticles to match the other material. Some approaches include introducing a block copolymer, capping the polymer chain ends with hydrophilic groups, and modifying the polymer side chains with hydrophilic groups. ${ }^{[15-18]}$ An alternative solution is surface modifying the outside of the nanoparticle with a small bridging ligand so that the nanoparticles can physically attach to the polymer. These ligands can be divided into aliphatic ligands or aromatic ligands. Aliphatic ligands contain a reactive side group that chemically binds to the inorganic particle and a long hydrocarbon tail intended to interact preferably with the polymer hydrocarbon side chain. Side groups that have shown successful chemical bonding to $\mathrm{ZnO}$ or $\mathrm{TiO}_{2}$ nanorods include thiols, carboxylic acids, phosphonic acids, silanes, and siloxanes. ${ }^{[19-25]}$ Aromatic ligands also contain a reactive side group to bind to the nanoparticle, but instead of a hydrocarbon chain, contain a large conjugated system that can extend much farther outwards than aliphatic ligands. Aromatic ligands including porphyrins, inorganic dyes, buckminsterfullerene, and conjugated oligomers have all 
been attached to the nanowire surface with similar side groups. ${ }^{[32-38]}$ Aromatic ligands are generally more complicated to synthesize or acquire than aliphatic ligands, but they accomplish multiple goals in the end electronic device. These ligands can increase the miscibility of the nanoparticle and polymer similar to aliphatic ligands, but they can also contribute to charge transfer. The conjugated system essentially creates a continuation of the polymer backbone and allows excitons to travel more easily to the inorganic/organic interface as opposed to the unconjugated hydrocarbon chain in aliphatic ligands. The conduction band of the ligands also allows holes from separated electron-hole pairs to travel along the conjugated chain much like the polymer donor material. It was found that an aromatic phenyl ligand increased the short circuit current density of a hybrid photovoltaic device more than one fold over an aliphatic propyl ligand due to efficient charge transfer dynamics at the interface. ${ }^{[39]}$ Figure 1.7 highlights the influence of ligand type and size on the aggregation behavior of inorganic nanowires. 
(a)

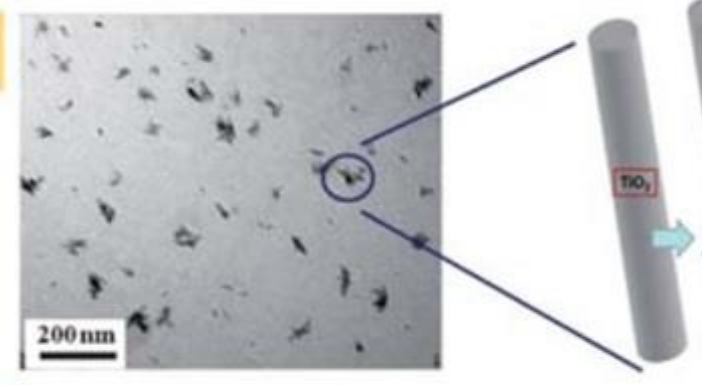

(b)
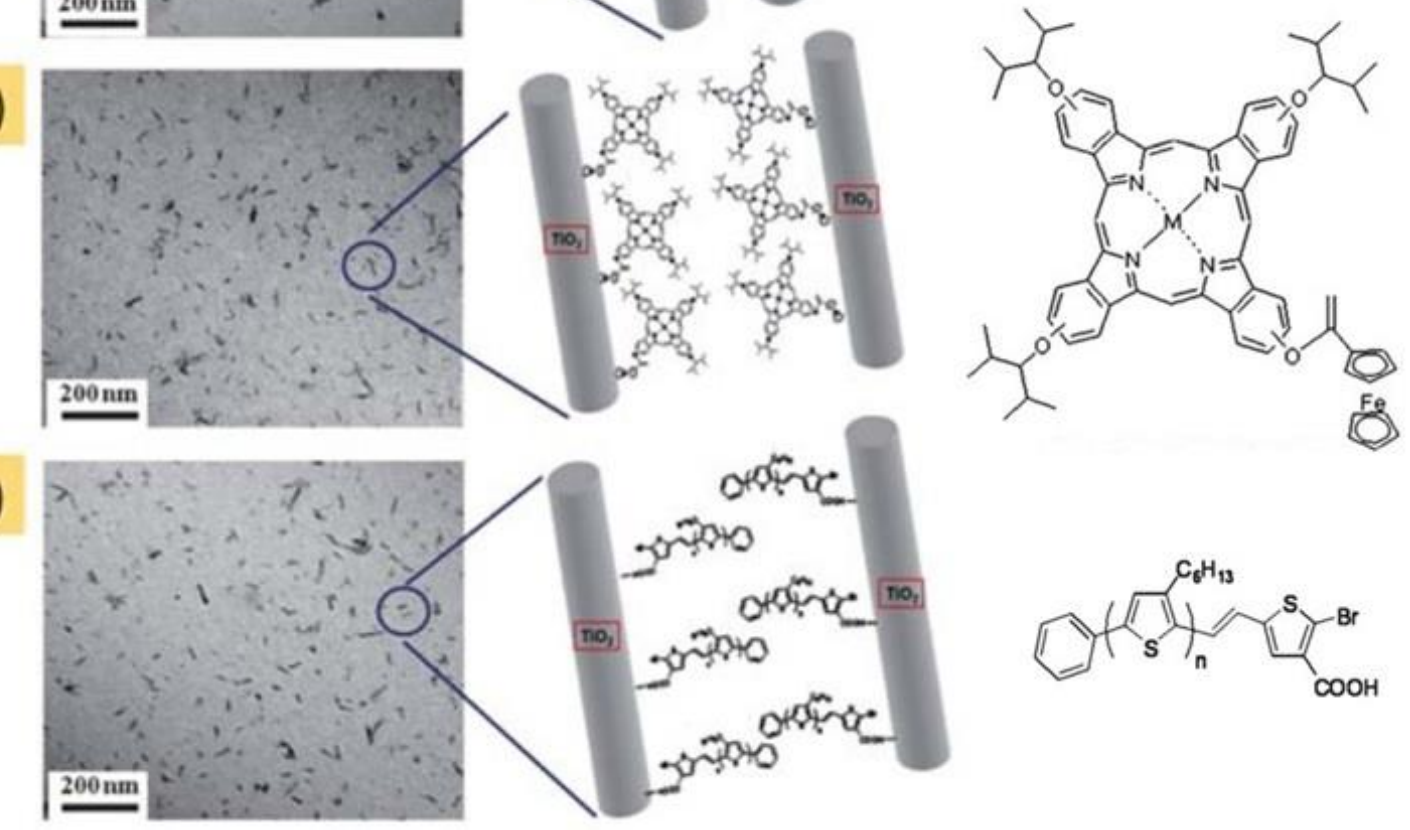

(c)

Figure 1.7. Tunneling electron microscopic (TEM) and cartoon images of $\mathrm{TiO}_{2}$ nanorods surface modified with (a) pyridine, (b) a Cu-phthalocyanine-ether dye, and (c) Oligo$3 \mathrm{HT}-(\mathrm{Br}) \mathrm{COOH}, \mathrm{n} \approx 27$. The tendency of the nanorods to aggregate is decreased with long conjugated ligands. ${ }^{[34]}$

Isotropic nanostructures can also be incorporated between the organic/inorganic interface through modification procedures. As stated before, quantum dots have been shown to increase device efficiency by generating excitons themselves and providing a more direct electron injection pathway into neighboring materials. However, quantum dots are not dense enough to create a continuous layer themselves as the inorganic component in hybrid devices, so much research has gone into anchoring the dots onto other particles. ${ }^{[31,40-43]}$ A common approach is anchoring the dots onto an inorganic 
nanorod either by direct grafting or via a ligand. Figure 1.8 displays a successful coating of $\mathrm{CdS}$ quantum dots onto $\mathrm{TiO}_{2}$ nanowire, by directly growing the dots onto the nanowire in situ. Compared to the bare nanowires, the $\mathrm{CdS} / \mathrm{TiO}_{2}$ nanoassemblies showed an enhancement in photocurrent efficiency of $300 \%$, while the $\mathrm{CdTeS} / \mathrm{TiO}_{2}$ showed an improvement of $350 \% .^{[31]}$

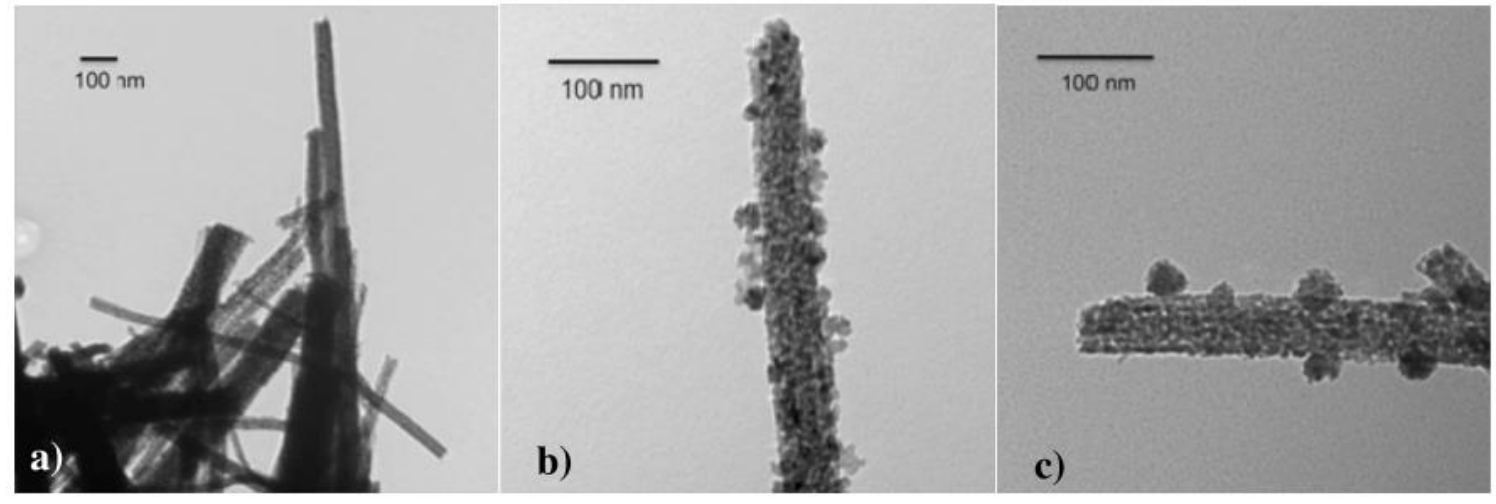

Figure 1.8. TEM images of (a) $\mathrm{TiO}_{2}$ nanowires, (b) $\mathrm{CdS}$ coated $\mathrm{TiO}_{2}$ nanowires, and (c) CdTeS coated $\mathrm{TiO}_{2}$ nanowires. ${ }^{[31]}$

\subsection{Conjugated Semi-conducting Polymers}

Letheby's discovery in 1862 revealed the possibility of a new family of flexible conductive plastics. Yet this achievement went largely unnoticed, as the scientific community still understood very little about the nature of macromolecular chains. In the early 1920's, researchers started to unearth the chemical structure of CPs, and their usefulness became apparent in electronic applications. ${ }^{[44]}$ Several polymers seen in Figure 1.9 were found to exhibit semi-conductive properties due to their conjugation of alternating carbon single bond-double bond backbone. The $\pi$ electrons in these double bonds are delocalized and thus the valence electrons in the system have a high mobility along the backbone. 

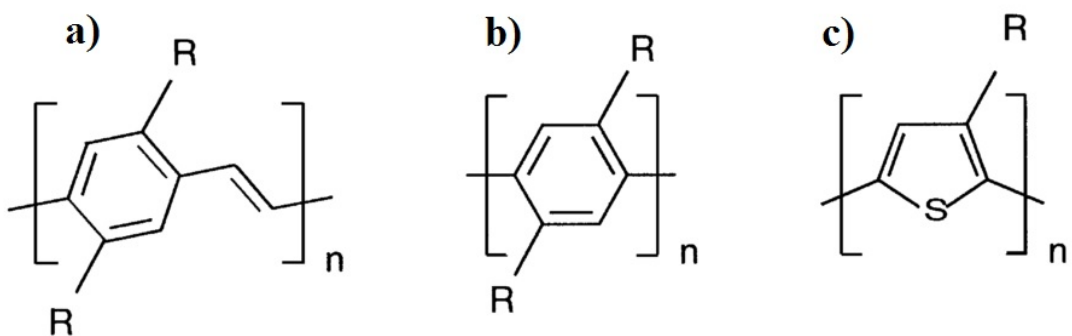

Figure 1.9. Chemical structures of common semiconducting CPs. (a)

poly(phenylenevinylene), (b) poly(phenylene), and (c) poly(thiophene). ${ }^{[44]}$

Mathematically, the polymer chain can be treated as a quantum mechanical particle in a box system with energy $E_{n}$ given by Equation 1.2.

$$
E_{n}=\frac{n^{2} h^{2}}{8 m_{e} L^{2}}
$$

Where, $\mathrm{n}$ is the electron's energy level, $\mathrm{h}$ is Planck's constant, $\mathrm{m}_{\mathrm{e}}$ is the mass of an electron, and $\mathrm{L}$ is the length of the box. The energy is inversely proportional to the length of the box, or in this case the length of the backbone polymer chain. The electrons in the conjugated system in their ground state collectively create a HOMO energy level referred to as the valence band. These electrons can be excited into a higher energy band referred to as the conduction band seen in Figure 1.10. 


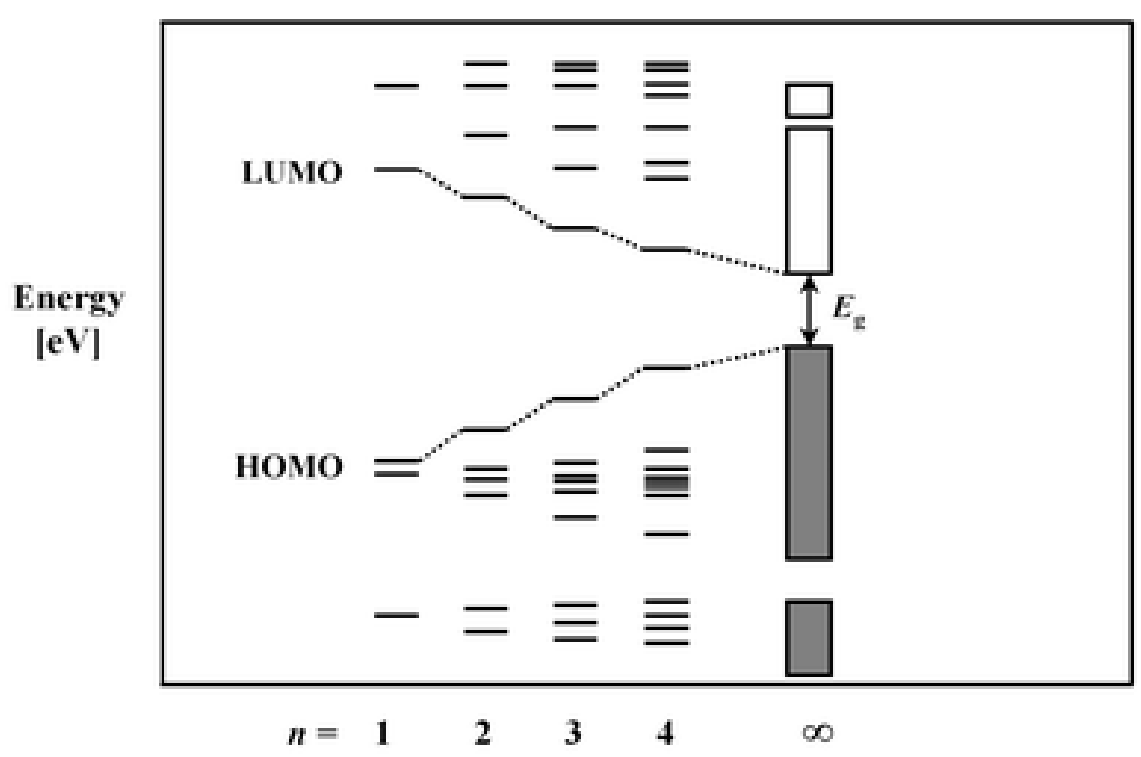

Figure 1.10. Diagram of HOMO and LUMO energy levels in polymer, where at infinite chain length a valence band and conduction band are formed. ${ }^{[45]}$

The band gap is defined as the energy difference between the two bands, and is essentially the amount of energy required to mobilize an electron in the system. In a solar cell it would be the energy of the incoming photon. The energy of this band gap is then defined by Equation 1.3.

$$
\Delta E=E_{\text {LUMO }}-E_{\text {HOMO }}=\frac{\left(n_{\text {LUMO }}-n_{\text {HOMO }}\right) h^{2}}{8 m_{e} L^{2}}
$$

This equation highlights the tailorability of CPs as semiconducting materials, because as the length of the chain increases, the band gap decreases. The location of the valence and conduction bands is also dependent on a variety of other factors, including temperature, solvent and doping. However, the most important factor for the present work are the inter- and intramolecular interactions due to neighboring polymer chains.

In the case of the CP poly(3-hexylthiophene) (P3HT), the conformation of the chains will have a dramatic effect on the band gap energy and because the polymer's band gap lies near the visible region this creates a change in color, as seen in Figure 1.11. 

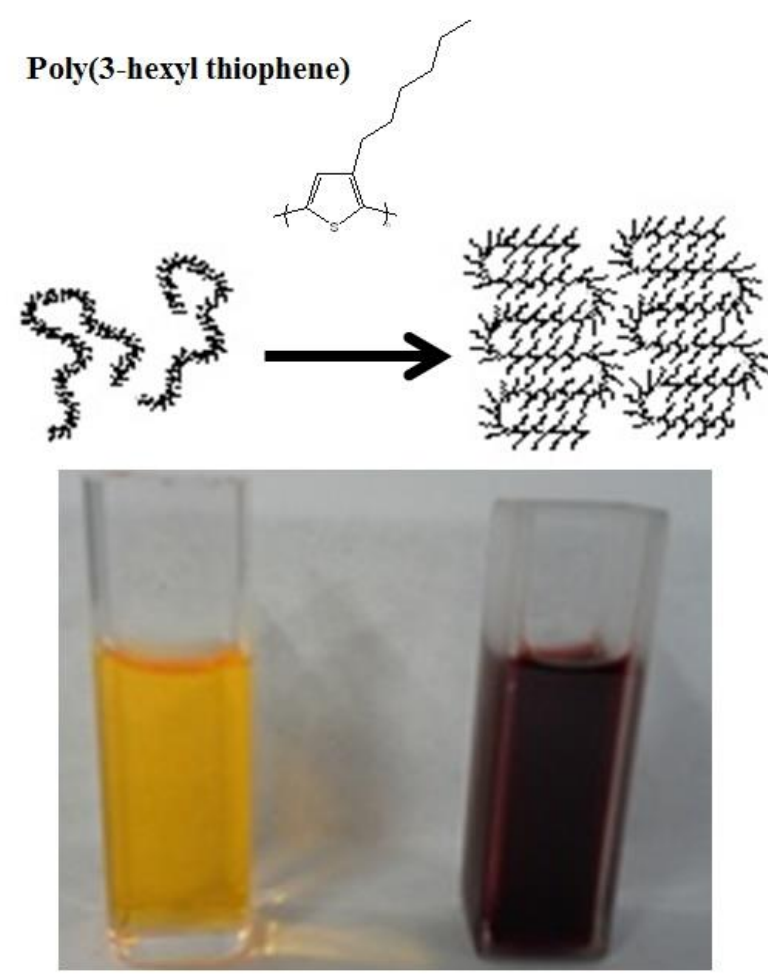

Figure 1.11. Graphic depicting the effect of intramolecular chain interaction on P3HT in solution (anisole).

In a good solvent, $\mathrm{P} 3 \mathrm{HT}$ exists in a solvated coil-like conformation due to favorable polymer-solvent interactions which maximize the chain surface area. In a poor solvent, P3HT can exhibit either a coil-like conformation or a rod-like conformation. At higher temperatures, the polymer is entropically dominated and exhibits the coil conformation. But at lower temperatures, the polymer is enthalpically dominated and favors a rod conformation, which minimizes the polymer-solvent interactions. In this rodlike conformation, the polymer chains interact more with each other in a pi-pi stacking fashion. The conjugated pi electrons in the thiophene ring line up with neighboring rings aligning the polymer chains. In solution this pi-pi stacking occurs through a combination of two different processes. The first is through intramolecular chain folding through polymer crystallization. At lower temperatures the polymer chain will minimize polymer- 
solvent interaction by folding in on itself creating a lamellar crystal structure, as seen in Figure 1.12. The second process that brings polymer chains in closer contact is an intermolecular force of aggregation. Multiple chains will aggregate together at lower temperatures, and this lowers the polymer-solvent interactions and increases the proximity of neighboring chains. Again, these two processes are temperature dependent, so as the P3HT solution is heated or cooled it will display a characteristic color change. This is the basis behind P3HT thermochromism, and is a fundamental tool in analyzing the morphology of polymer used in hybrid devices.

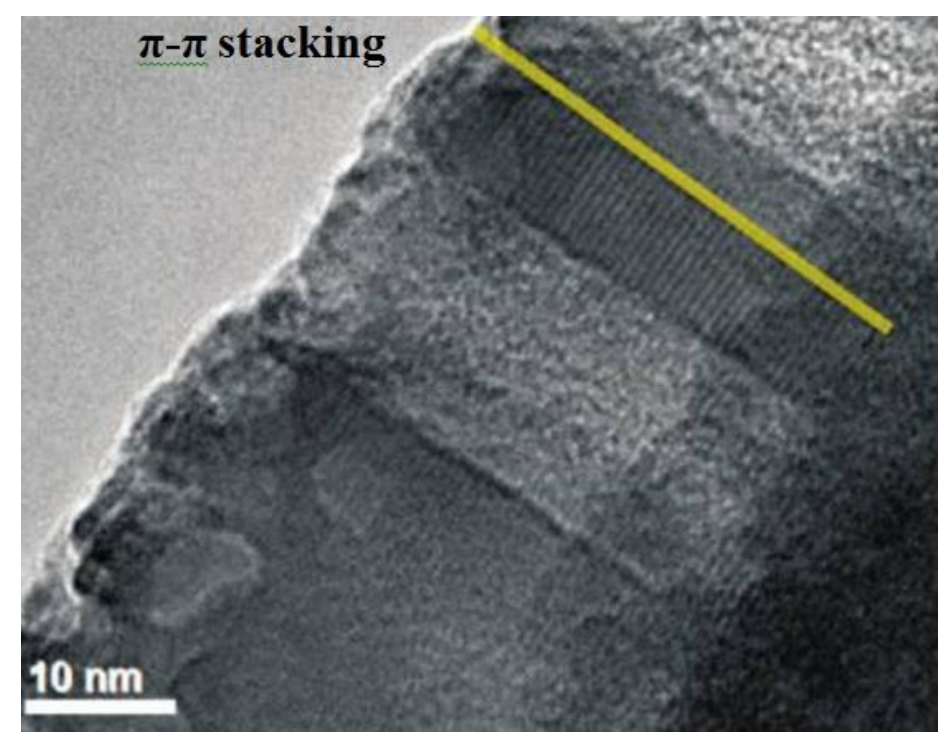

Figure 1.12. TEM image of crystallized P3HT on a CdS nanowire surface. ${ }^{[46]}$

The conformation and morphology of the polymer in a hybrid device is critical to understand because it not only corresponds to the band gap, but also to the efficiency of charge transport. Because of the quantum mechanical nature of charge carriers, they can travel both along the polymer chain through conduction transport, or they can hop between adjacent chains through hopping transport, seen in Figure 1.13. Hopping transport is typically much slower than conduction transport. Here, the rigidity of the 
chains and the proximity of chains to one another are incredibly important. In terms of conformation, a coil-like polymer retains a large free volume, so the distance between chains is farther than in the rod-like state. Aggregation and crystallized chain-folded polymer will allow chains to be in more intimate contact, which is ideal for both transport mechanisms. However, aggregation of polymer chains in a hybrid device will also cause phase separation and have an overall tendency to lower device efficiency. So in terms of transport efficiency in hybrid electronics, the ideal polymer layer is one that requires the least amount of energy to crystallize and form lamellar, rigid microstructures.

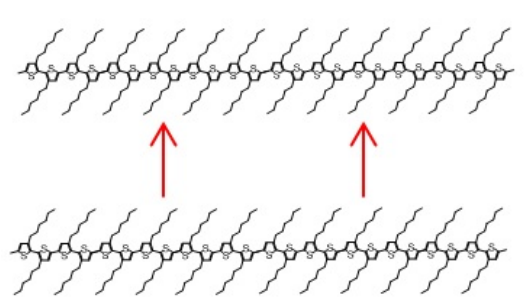

Hopping transport
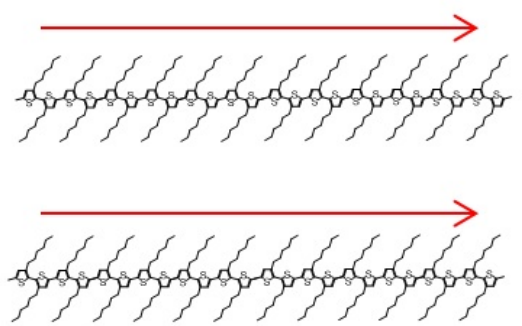

Conduction transport

Figure 1.13. Direction of hopping transport and conduction transport in P3HT. 


\subsection{Polymer Crystallization}

Polymer crystallization is one of the more complicated topics in polymer science because of the unique behavior of polymer chains during phase transitions. It is helpful to start with a visualization of the polymer crystallization process, which can occur either with pure polymer in the melt, or with polymer dissolved in solution. In terms of polymer models that help visualize the process, there are no differences between melt or solution crystallization, but these differences will have an influence on the thermodynamic equations describing the models. Polymer crystallization can be classified into three groups: crystallization during polymerization, crystallization induced by orientation, and crystallization under quiescent condition. ${ }^{[47-48]}$ Some crystallization behavior of P3HT in solution could potentially be described by orientation, but the largest contributor is crystallization behavior under quiescent conditions. Figure 1.14 shows a conceptual map of the four different models proposed to describe the complex nature of polymer crystallization.

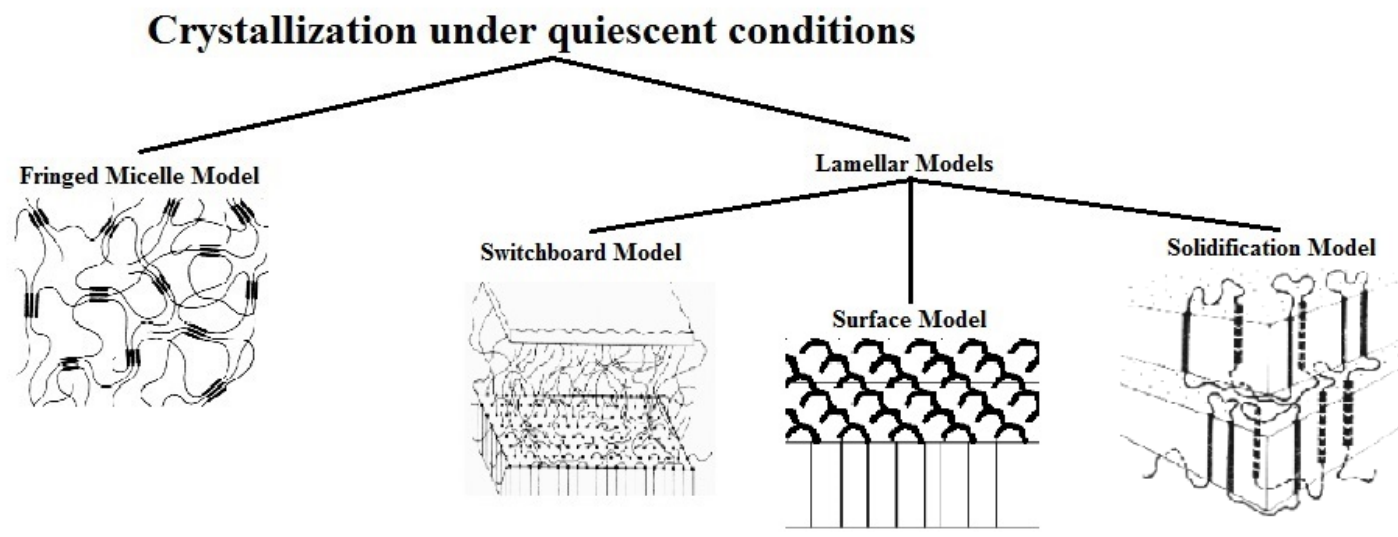

Figure 1.14. Types of models proposed for polymer crystallization under quiescent conditions. $^{[49]}$ 
The fringed micelle model suggests that segments of polymer chains form straight segments next to other polymer chain segments, forming regions of uniform crystallinity that act as physical crosslinks. ${ }^{[50-51]}$ This model has been proven accurate for certain polymers that crystallize through rapid cooling/quenching from the melt, but the majority of polymers have been found to exhibit lamellar microstructures, leading to several lamellar models. ${ }^{[52]}$ The surface model is an idealized lamellar structure where single chains crystallize, bend, and undergo adjacent reentry creating a sharp surface boundary ${ }^{[53]}$ The switchboard model is more of a random interpretation of the process, where single chains crystallize, then form long amorphous segments and reenter the same lamella or a nearby lamella randomly forming an entangled system. ${ }^{[52,54-55]}$ The solidification model or "Erstarrungsmodell" is perhaps the most complex, but accurate depiction of polymer crystallization. Here, individual polymer chains form crystalline regions first and then are fit into lamellae without significant reorganization. ${ }^{[56]}$

The type of crystallization behavior that is observed in reality is dependent on the polymer type, concentration, and molecular weight. Polymer crystallization rarely matches one type of model exactly, and is in reality a statistical mix of several models. Any particular polymer will exhibit behavior similar to all of the four principal models, and thus describing the exact process as a whole is nearly impossible. In order to develop thermodynamic equations, most theories refer to the lamellar surface model, which incorporates a uniform lamella structure and adjacent reentry.

The polymer crystallization process can also influence or be influenced by other polymer-polymer interactions like conformational changes. In the example of cooling P3HT in a poor solvent, the coil to rod conformational transition and chain aggregation 
occur alongside crystallization as seen in Figure 1.15. It is believed that the conformational transition from coil to rod initiates P3HT crystallization during solventinduced precipitation. ${ }^{[57]}$ Thus, P3HT crystallization is really a combination of multiple transitions. The color change of $\mathrm{P} 3 \mathrm{HT}$ as it undergoes crystallization is a result of the sum of chain folding and aggregation. While these two processes cannot easily be distinguished, the overall rate of crystallization can provide useful information about how energetically favorable it is for the polymer to align with itself.

\section{P3HT Crystallization Process}

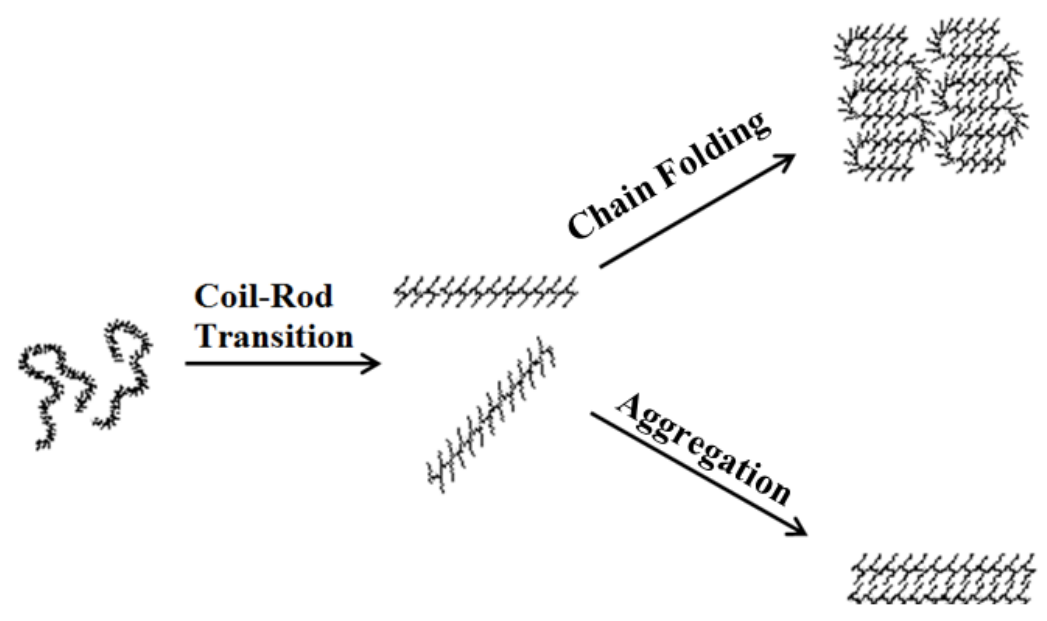

Figure 1.15. Scheme of the steps involved in the crystallization of P3HT.

\subsection{Kinetics of Polymer Crystallization}

Polymer crystallization is a physical phase change, and thus its thermodynamics and kinetics can be analyzed similar to dynamic processes like chemical reactions. Chemical reactions and phase changes typically obey specific principles, such as rate laws. The overall rate of change that a reaction undergoes is given by:

$$
\text { Rate }=k[\text { reactant }]^{\alpha}
$$


Where $\mathrm{k}$ is the rate constant, [reactant] is the concentration of reactant and $\alpha$ is the reaction order. $\mathrm{k}$ is a constant that is dependent on many external and internal factors like catalysts, temperature and solvent. The $\alpha$ term is representative of the reaction mechanism and gives some idea of how the reaction occurs on a molecular level. ${ }^{[58]}$

$$
\log (\text { Rate })=\log (k)+\alpha \cdot \log [\text { reactant }]
$$

Taking the logarithm of Equation 1.4 yields Equation 1.5, which gives a useful relationship between the $\log ($ Rate $)$ and $\log [$ reactant $]$. Both Equations 1.4 and 1.5 can be incorporated into the method of initial rates, where samples of varying concentration are measured over a short time and their initial reaction rates are obtained. Plotting the data with Equation 1.5 should give a straight line of slope $\alpha$, revealing information about the reaction mechanism. While some polymers crystallize in a manner that can be analyzed through simple reaction rate kinetics, others have more complex behavior because of their crystallization mechanisms. Several thermodynamic approaches to polymer crystallization have emerged including the Gibbs-Thomson equation, Avrami analysis, and Lauritzen-Hoffman Growth Theory.

Avrami analysis relates very closely to rate law kinetics, in that the theory focuses on the change in crystallization behavior as crystallization occurs isothermally. This is in contrast to the method of initial rates, which compares the temperature dependence of initial polymer crystallization. It is important to note the differences between these two methods, as they provide similar constants in the analysis, but these constants provide significantly different information about the crystallization process. For instance, the rate constant $\mathrm{k}$ in rate law analysis (method of initial rates) describes the change in initial crystallization rate of polymer as a function of the concentration of amorphous polymer 
in solution. This value describes the nucleation behavior of the polymer and how a higher concentration of uncrystallized polymer around the nucleated site affects this process. In contrast, the kinetic growth rate constant $\mathrm{k}$ in Avrami analysis describes the change in crystallization rate during the crystallization of one polymer solution. This value describes the change in polymer crystallization rate as the surrounding solution becomes more crystalline. The Avrami model follows equation (1.6)

$$
1-X=e^{-k t^{n}}
$$

Where $\mathrm{X}$ is the relative percent crystallinity of polymer, $\mathrm{k}$ is the kinetic growth rate constant, and $\mathrm{n}$ is the Avrami exponent. $\mathrm{k}$ is also referred to as the bulk crystallization constant and $\mathrm{n}$ is mostly referred to as the Avrami constant or Avrami exponent. The Avrami exponent is related to the type of nucleation and to the geometry of the growing crystals. The Avrami exponent can yield information on the dimensionality of crystal growth, and more specifically whether the polymer crystals more resemble spheres, discs, or rods. ${ }^{[59-62]}$ Here, $\mathrm{X}$ can be treated as a relative normalized value where the initial value of crystallized polymer is 0 and the final amount of crystallized polymer is 1 . Taking the logarithm of Equation 1.6 yields a more useful relationship.

$$
\ln [-\ln (1-X)]=\ln (k)+n \cdot \ln (t)
$$

Here, the crystallization of a single polymer solution can be analyzed across its lifetime to provide a linear plot of $\ln [-\ln (1-\mathrm{X})]$ against $\ln (\mathrm{t})$. The best fit line will provide the parameters $\mathrm{k}$ and $\mathrm{n}$.

John D. Hoffman and coworkers initially proposed a simplified model of the polymer crystallization process, now known as Hoffman Nucleation Theory. ${ }^{[63-64]}$ 
The theory was later adapted by John Lauritzen and modified into a unifying theory that attempts to quantify and describe polymer crystallization kinetics via thermodynamics. The model begins with an amorphous polymer segment attaching to a substrate via secondary nucleation, seen in Figure 1.16.

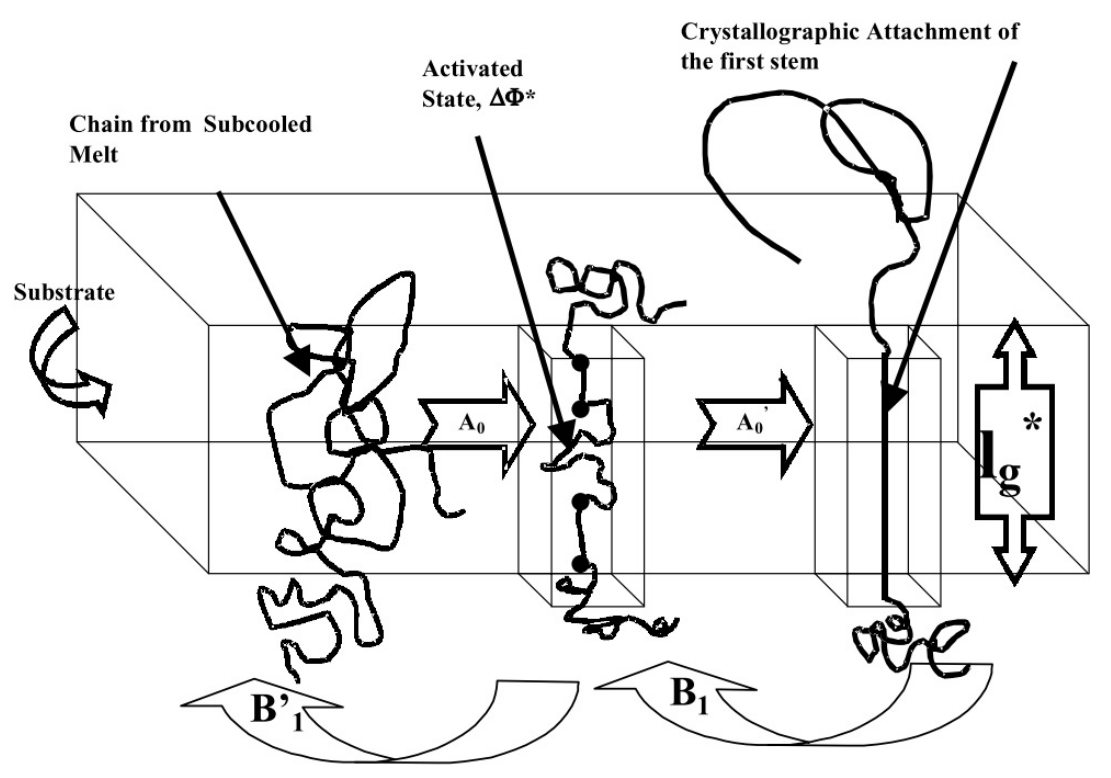

Figure 1.16. Schematic of initial stem deposition in polymer crystallization. ${ }^{[49]} \mathrm{A}_{0}$ and $\mathrm{A}_{0}$ ' are the forward reaction rates of stem deposition, $\mathrm{B}_{1}$ and $\mathrm{B}_{1}$ ' are the reverse reaction rates of stem deposition, $1_{\mathrm{g}}{ }^{*}$ is the average crystal stem length.

During the beginning stages of polymer crystallization, the chains can be assumed to be completely amorphous. The substrate in this case can be some type of other crystallized polymer, a nucleating particle, or contaminate. In any case, the initial portion of polymer that deposits onto the surface to create an activated state $\Delta \Phi^{*}$ is called the stem. The rates of the stem attaching to and elongating onto the substrate surface are denoted $\mathrm{A}_{0}$ and $\mathrm{A}_{0}$, while the reverse reaction rates are denoted $\mathrm{B}_{1}$ and $\mathrm{B}_{1}$ '. 


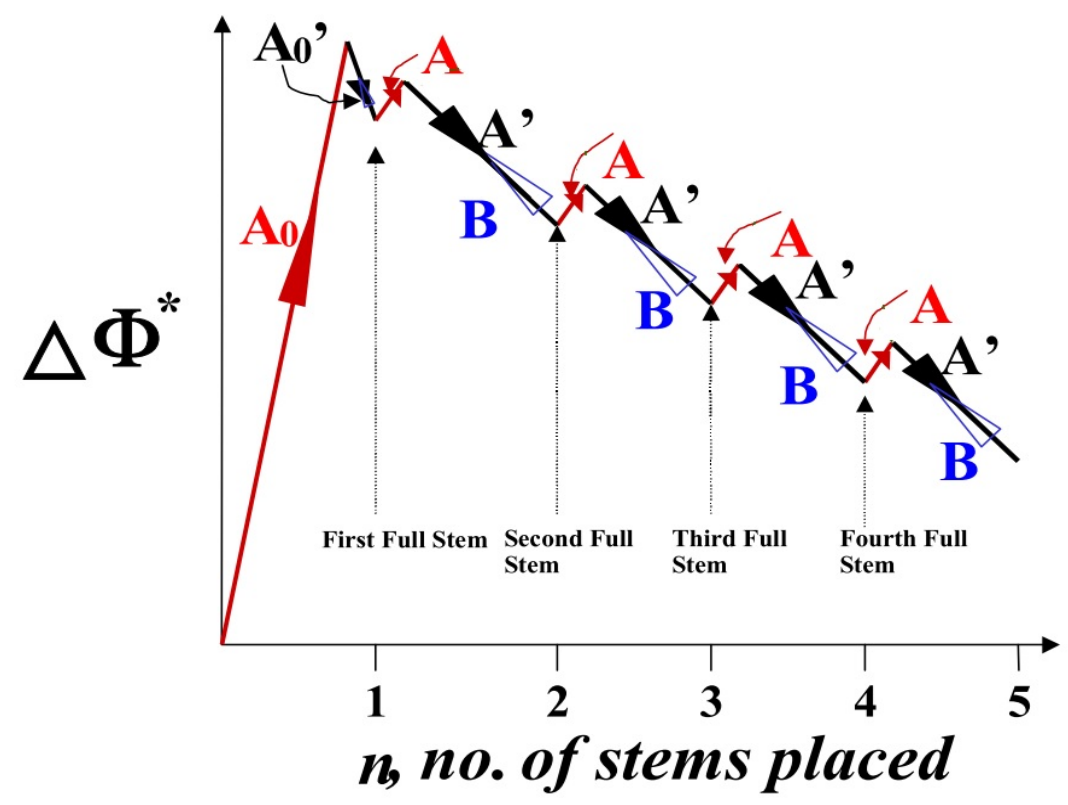

Figure 1.17. Falling activation energy of subsequent polymer chain reentry. ${ }^{[49]}$

The initial energy barrier for stem deposition is large because of the relative change in surface energy during the process. Once the initial stem has been deposited, the energy barrier associated with continued crystallization is much lower and thus lamella formation is favorable. By using steady state flux calculations the rate of stem deposition is given by: ${ }^{[65]}$

$$
S=\frac{N_{0} A_{0}(A-B)}{A-B+B_{1}}
$$

Where $\mathrm{N}_{0}$ is the number of initial stems and:

$$
\begin{gathered}
A_{0}=\beta^{\prime} e^{\frac{-2 b_{0} l \sigma+\psi{ }^{\prime} a_{0} b_{0} l \Delta G_{f}}{k T_{c}}} \\
B_{1}=\beta^{\prime} e^{\frac{-(1-\psi \prime) a_{0} b_{0} l \Delta G_{f}}{k T_{c}}} \\
A=\beta e^{\frac{-2 b_{0} l \sigma_{e}+\psi a_{0} b_{0} l \Delta G_{f}}{k T_{c}}} \\
B=\beta e^{\frac{-(1-\psi) a_{0} b_{0} l \Delta G_{f}}{k T_{c}}}
\end{gathered}
$$


Where $b_{0}$ is the initial stem width, 1 is the initial stem length, $a_{0}$ is the initial stem height, $\mathrm{k}$ is Boltzmann's constant, $\psi$ is an apportionment factor and $\mathrm{T}_{\mathrm{c}}$ is crystallization temperature. $\beta$ is a pre-exponential factor that will be explained further later. Traditional (L-H) Theory treats $\beta=\beta$ ' and $\psi=\psi$ ' mainly due to the difficulty in finding a solution when the factors are not equal. ${ }^{[66]}$

Equations $1.8-1.12$ allow us to calculate the nucleation rate $\mathrm{i}$ by the relation: ${ }^{[63]}$

$$
i=\frac{1}{n_{i} a_{0} l_{u}} \int_{2 \sigma_{e} / \Delta G_{f}}^{\infty} S d l
$$

Where $n_{i}$ is the number of stems of width $a_{o}$ and length $1_{u}$. Substituting Equations 1.8 - 1.12 into Equation 1.13 provides a non-trivial integration which produces Equation 1.12 .

$$
i=\frac{N_{0} \beta}{n_{i} a_{0} l_{u}}\left[\frac{k T_{c}}{2 b_{0} \sigma}-\frac{k T_{C}}{2 b_{0} \sigma+\Delta G_{f}}\right] e^{\frac{-4 b \sigma \sigma_{e}}{\Delta G_{f} k T_{c}}}
$$

Where $T_{c}$ is the crystallization temperature and $b$ is the average stem width. This result gives us the rate of nucleation onto the substrate surface, but to determine the overall rate of crystal growth $\mathrm{G}$, we must also consider the rate of growth along the substrate surface g. There are three different possibilities, or regimes, for crystal growth based on the relative order of $\mathrm{i}$ compared to $\mathrm{g}$, shown in Figure 1.18. 

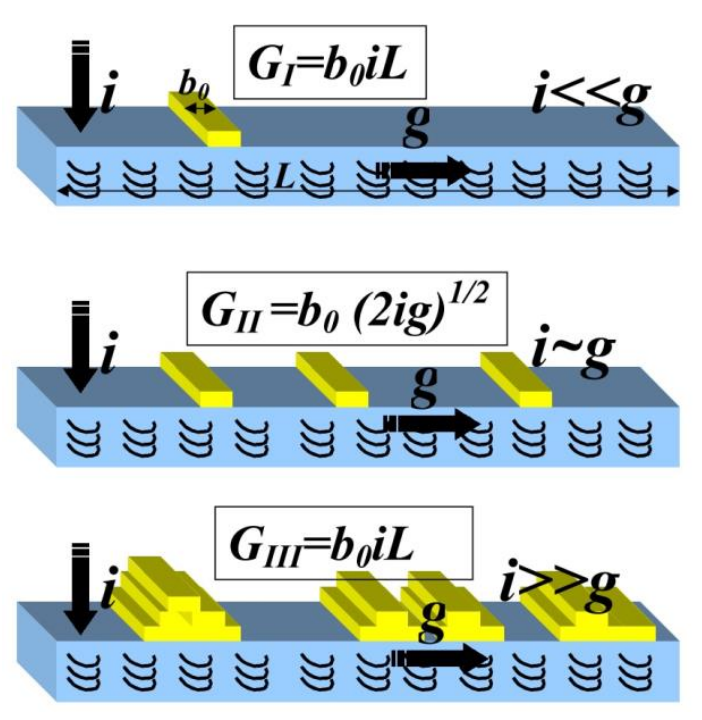

Figure 1.18. Schematic of the different regimes involved in polymer crystallization. ${ }^{[49]}$

The first regime occurs when $\mathrm{i}<<\mathrm{g}$, so the rate at which stems attach to the substrate surface is the limiting step, and once a stem is attached the subsequent crystallization of that chain is fast. Because the nucleation rate is the limiting step the total growth rate $\mathrm{G}$ is proportional to $\mathrm{i}$. In the second regime $\mathrm{i} \approx \mathrm{g}$, so the overall rate becomes a combination of both $\mathrm{i}$ and $\mathrm{g}$. In the final regime $\mathrm{i}>>\mathrm{g}$, which means the rate of step deposition is so much larger than the propagation of adjacent chains, that stems continue to deposit on top of other stems before layers can be completed. In this case, the outward growth of the crystal is dependent only on the nucleation rate $\mathrm{i}$, similar to that of regime I. These regimes have been experimentally observed and confirmed for a large number of polymers. ${ }^{[66]}$ The substrate completion rate $\mathrm{g}$ is defined in Equation 1.15, but because we will only be dealing with regime I kinetics, this rate will not be discussed further.

$$
g=a_{0} \beta\left[1-e^{\frac{a_{0} b_{0} \delta l \Delta G_{f}}{k T_{c}}}\right] e^{\frac{-2 a_{0} b_{0} \sigma_{e}}{k T_{c}}}
$$


In regime I, the overall growth rate of the polymer crystal is given by

Equation 1.16.

$$
G_{I}=b_{0} i L
$$

Where $\mathrm{L}$ is the length of the entire substrate. We can now substitute the nucleation rate i from Equation 1.14 into Equation 1.16 and simplify to find an expression for the growth rate.

$$
G_{I}=\frac{b_{0} L N_{0} \beta}{n_{1} a_{0} l_{u}}\left[\frac{k T_{c}}{2 b_{0} \sigma}-\frac{k T_{c}}{2 b_{0} \sigma+\Delta G_{f}}\right] e^{\frac{-4 b_{0} \sigma \sigma_{e}}{\Delta G_{f} k T_{c}}}
$$

Where

$$
\beta=J e^{\frac{-U^{*}}{R\left(T_{C}-T_{\infty}\right)}}
$$

and

$$
J=\frac{\kappa}{n}\left(\frac{k T_{c}}{h}\right)
$$

Here the pre-exponential factor $\beta$ is broken down into another pre-exponential factor $\mathrm{J}$ and an exponential where $\mathrm{U}^{*}$ is the activation energy of deposition, $\mathrm{R}$ is the ideal gas constant, and $\mathrm{T}_{\infty}$ is equal to the glass transition temperature $\mathrm{T}_{\mathrm{g}}-30^{\circ}$. The second preexponential factor $\mathrm{J}$ is expressed in terms of $\mathrm{kT}_{\mathrm{c}} / \mathrm{h}$ the frequency factor in events per second. $\mathrm{n}$ is the number of repeat units of polymer and $\kappa$ is a numerical constant which is evaluated from the monomeric friction coefficient. Equation 1.17 can be simplified into the following form:

$$
G_{I}=\frac{b_{0} N_{0} J}{l_{u}}\left[\frac{k T_{c}}{2 b_{0} \sigma}-\frac{k T_{c}}{2 b_{0} \sigma+\Delta G_{f}}\right] e^{\frac{-U^{*}}{R\left(T_{c}-T_{\infty}\right)}} e^{\frac{-4 b_{0} \sigma \sigma_{e}}{\Delta G_{f} k T_{c}}}
$$

Here it is useful to simplify the entire pre-=exponential into a convenient constant denoted $\mathrm{G}_{0}$. 


$$
G_{I}=G_{0} e^{\frac{-U^{*}}{R\left(T_{c}-T_{\infty}\right)}} e^{\frac{-4 b_{0} \sigma \sigma_{e}}{\Delta G_{f} k T_{c}}}
$$

Equation 1.19 is perhaps the most useful equation in (LH) Theory because it sums up the entire process of polymer crystallization. The $\mathrm{G}_{0}$ term describes the change in surface energy of a layer of polymer crystal taking into account the polymer dimensions and the frequency of events. The second exponential term describes the homogeneous nucleation, where $\mathrm{U}^{*}$ is the energy required for "reeling" in the polymer chain to form crystalline regions. This is believed to occur mainly by a mechanism of steady state reptation. ${ }^{[67]}$ The second exponential describes the heterogeneous nucleation, which in dilute solution becomes the most important factor. This is because in dilute solution the side-to-side motion of the chain, which is allowed by the presence of solvent molecules will eliminate the possibility of reptation. ${ }^{[67]}$ Thus, the first exponential term can be treated as a constant in dilute solution experiments over a small $\Delta \mathrm{T}$ range of $\sim 30 \mathrm{~K}$, which is easily attainable in solution polymer kinetics studies. ${ }^{[67]}$ Also, the temperature dependence of the $\mathrm{G}_{0}$ is small enough that it can be treated as a constant also along the same $\Delta \mathrm{T}$ range. Taking the natural $\log$ of Equation 1.21 yields Equation 1.22.

$$
\ln \left(G_{I}\right)=\left[\ln G_{0}-\frac{U^{*}}{R\left(T_{c}-T_{\infty}\right)}\right]-\frac{4 b_{0} \sigma \sigma_{e}}{\Delta G_{f} k T_{c}}
$$

At this point it is useful to combine some of the variables in the final term. Typically the term $\mathrm{K}_{\mathrm{g}(\mathrm{i})}$ is used and is defined as the regime dependent nucleation constant.

$$
K_{g(i)}=\frac{4 b \sigma \sigma_{e} T_{d}^{0}}{k \Delta H_{f}}
$$

Where $\Delta \mathrm{H}_{\mathrm{f}}$ is the enthalpy of formation. In order to relate this we must use the definition of Gibbs free energy $\Delta \mathrm{G}_{\mathrm{f}}$ and its relation to the change in entropy $\Delta \mathrm{S}_{\mathrm{f}}$. 


$$
\Delta G_{f}=\Delta H_{f}-T \Delta S_{f}
$$

At the melting temperature $\mathrm{T}_{\mathrm{m}}{ }^{0}$, the change in the Gibbs free energy is 0 by definition so Equation 1.24 becomes:

$$
\frac{\Delta H_{f}}{T_{m}^{0}}=\Delta S
$$

By approximating this result for temperatures near the melting point we can substitute this back into Equation 1.24:

$$
\left[\Delta G_{f}=\Delta H_{f}-T \frac{\Delta H_{f}}{T_{m}^{0}}\right] \text { or }\left[\Delta G_{f}=\Delta H_{f}\left(\frac{T_{m}^{0}-T}{T_{m}^{0}}\right)\right]
$$

Now by substituting Equation 1.23 back into Equation 1.22 using these assumptions we develop the final equation in (LH) Theory. Note that L-H Theory is typically applied to melt crystallization, but as it is being adapted to solution crystallization, the $\mathrm{T}_{\mathrm{m}}{ }^{0}$ variable is directly converted to a $\mathrm{T}_{\mathrm{d}}{ }^{0}$, because these equilibrium transition temperatures are equivalent, but deal with two different contexts.

$$
\ln \left(G_{I}\right)=\left[\ln G_{0}-\frac{U^{*}}{R\left(T_{C}-T_{\infty}\right)}\right]-\frac{K_{g(i)}}{T_{c} \Delta T}
$$

Where $\left(\mathrm{T}_{\mathrm{m}}{ }^{0}-\mathrm{T}\right)$ is typically referred to as $\Delta \mathrm{T}$. Equation 1.27 is incredibly useful, because the portion in brackets is deemed constant, which leaves only the growth rate G, nucleation constant $\mathrm{K}_{\mathrm{g}(\mathrm{i})}$ and $\mathrm{T}_{\mathrm{c}}$ as variables. Experimentally, polymer crystal growth can be measured directly in the melt, or through another method such as spectroscopy or differential scanning calorimetry, to measure the growth rate of the polymer crystal at different crystallization temperatures. This produces a linear plot that reveals $\mathrm{K}_{\mathrm{g}(\mathrm{i})}$ as the slope. Figure 1.19 displays a typical L-H analysis of a polymer sample, highlighting the different slopes of $\mathrm{K}_{\mathrm{g}(\mathrm{i})}$. It is important to note that not all polymers display these regimes, 
and that because of the steep slope of $\mathrm{K}_{\mathrm{g}(\mathrm{II})}$ in regime II, the $\mathrm{T}_{\mathrm{c}}$ window for observing this regime is narrow.

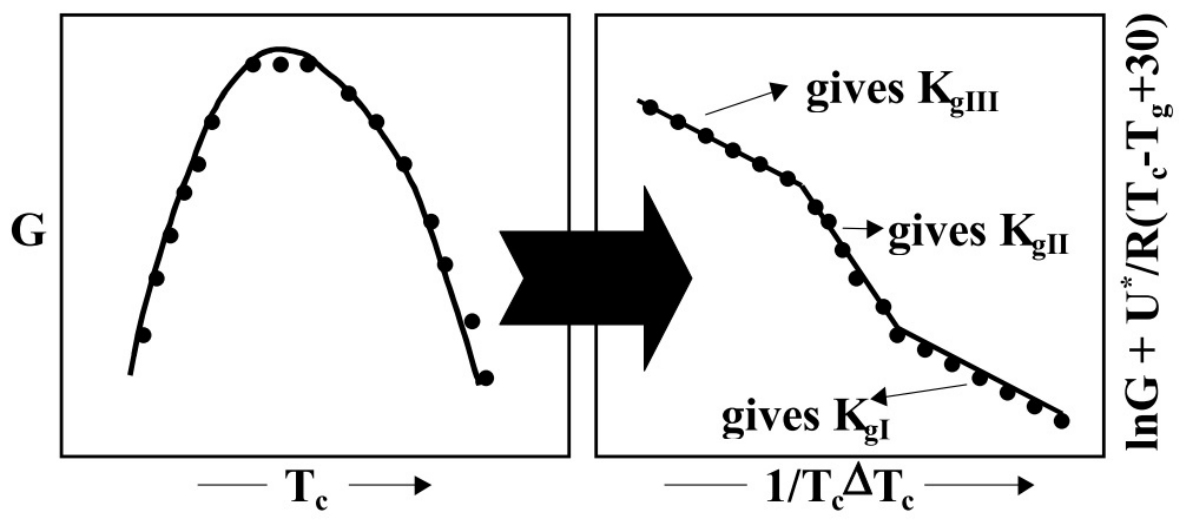

Figure 1.19. Typical L-H plots used to determine the regime boundaries and $\mathrm{Kg}$ values for polymer crystallization. ${ }^{[49]}$

The primary goal of this work is to determine the effect of modified nanoparticles on P3HT. L-H theory is a useful tool for analyzing the effect of these nanoparticles on the crystallization behavior of P3HT. Fluorescence spectroscopy is another tool used to analyze the interaction of the materials, namely potential for electron transfer.

Fluorescence spectroscopy involves shining light of a certain excitation wavelength on a sample and then detecting the emission spectrum at a $90^{\circ}$ angle. In the case of hybrid systems, usually both components have a unique emission spectrum at a given excitation wavelength. Inorganic nanoparticles in close proximity to polymer should theoretically "quench" this fluorescence due to charge transfer into the nanoparticle as a secondary path for the excited electrons. Thus, fluorescence quenching is a common indicator that nanoparticles in close enough proximity to polymer and are effectively allowing charge transport in between the two phases. 
This work will also extensively use UV-visible spectroscopy to analyze the thermodynamic behavior of P3HT with and without modified nanowire present. First, trends in the reaction mechanism of the systems will be explored by incorporating the thermochromism of the polymer during crystallization. Second, the $\mathrm{T}_{\mathrm{d}}{ }^{0}$ of the P3HTsolvent system is determined in order to employ L-H Theory with accurate parameters. Kinetics data of P3HT crystallization with and without modified $\mathrm{ZnO}$ is then analyzed through L-H theory to provide trends in surface folding energy to substantiate the effects of nanowire as a nucleation site. Quantum dots were then characterized via UV-visible and IR spectroscopy, and attempts were made to synthetically attach the dots to $\mathrm{ZnO}$ nanowire via ligands. Both quantum dots and modified $\mathrm{ZnO}$ were observed with P3HT using fluorescence spectroscopy to detect any possibility of quenching. Together, fluorescence spectroscopy, reaction rate kinetics, and L-H Theory provide a complete set of tools to understand the effect of inorganic nanoparticles on P3HT thermodynamics. 


\section{Experimental Methods}

\subsection{Material Preparation}

$$
\text { Regioregular Poly (3-hexylthiophene - 2,5-diyl) (P3HT, Rieke Specialty }
$$

Polymers Electronic Grade with molecular weight 50,000-70,000 ( $\left.\mathrm{g} \mathrm{mol}^{-1}\right)$ ), anisole (99\% pure, Acros Organics), chloroform, (99\%, Sigma Aldrich), ethanol (EtOH, Sigma Aldrich), dimethylsulfoxide (DMSO, Sigma Aldrich) were purchased from chemical suppliers and used without further purification. $\mathrm{ZnO}$ nanowires, shown in Figure 2.1 were prepared via solvothermal method outlined in Zhang et al. ${ }^{[23]}$

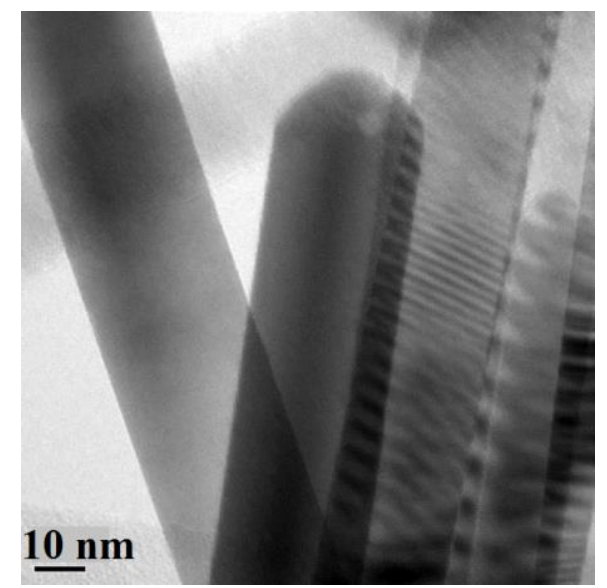

Figure 2.1. High-resolution TEM image of isolated pristine nanowires.

\subsubsection{Surface Modification of $\mathrm{ZnO}$ nanowire}

1-Dodecanethiol (DDT, 98\%, Acros Organics), mono-carboxylated tetraphenylporphyrin (TPP, provided by Dr. Chad Immoos, California Polytechnic State University San Luis Obispo) were used without further purification. A typical batch preparation used $20 \mathrm{mg} \mathrm{ZnO}$ nanowire. The $\mathrm{ZnO}$ white powder was dried in an oven at $60^{\circ} \mathrm{C}$ and pressed into a finer powder using a spatula. For DDT-modified $\mathrm{ZnO}$ and TPPmodified $\mathrm{ZnO}, 2.5 \mu \mathrm{L}$ DDT or $0.35 \mathrm{mg}$ TPP were added to every $1 \mathrm{mg} \mathrm{ZnO}$. The $\mathrm{ZnO}$ was added to a small centrifuge tube along with the DDT or TPP and filled to $1.5 \mathrm{~mL}$ 
with chloroform. The centrifuge tube was then vortexed over the course of 24 hours on a Fisher Scientific Digital Vortex Mixer. The supernatant of the samples were decanted, and the sample was refilled with chloroform and redispersed through shaking. Samples were then vortexed again and this rinsing process was repeated three times total. Samples were dried in an oven at $60^{\circ} \mathrm{C}$ for 2 hours and weighed. Samples were then either redispersed in anisole for UV-visible characterization or chloroform for IR spectroscopy characterization.

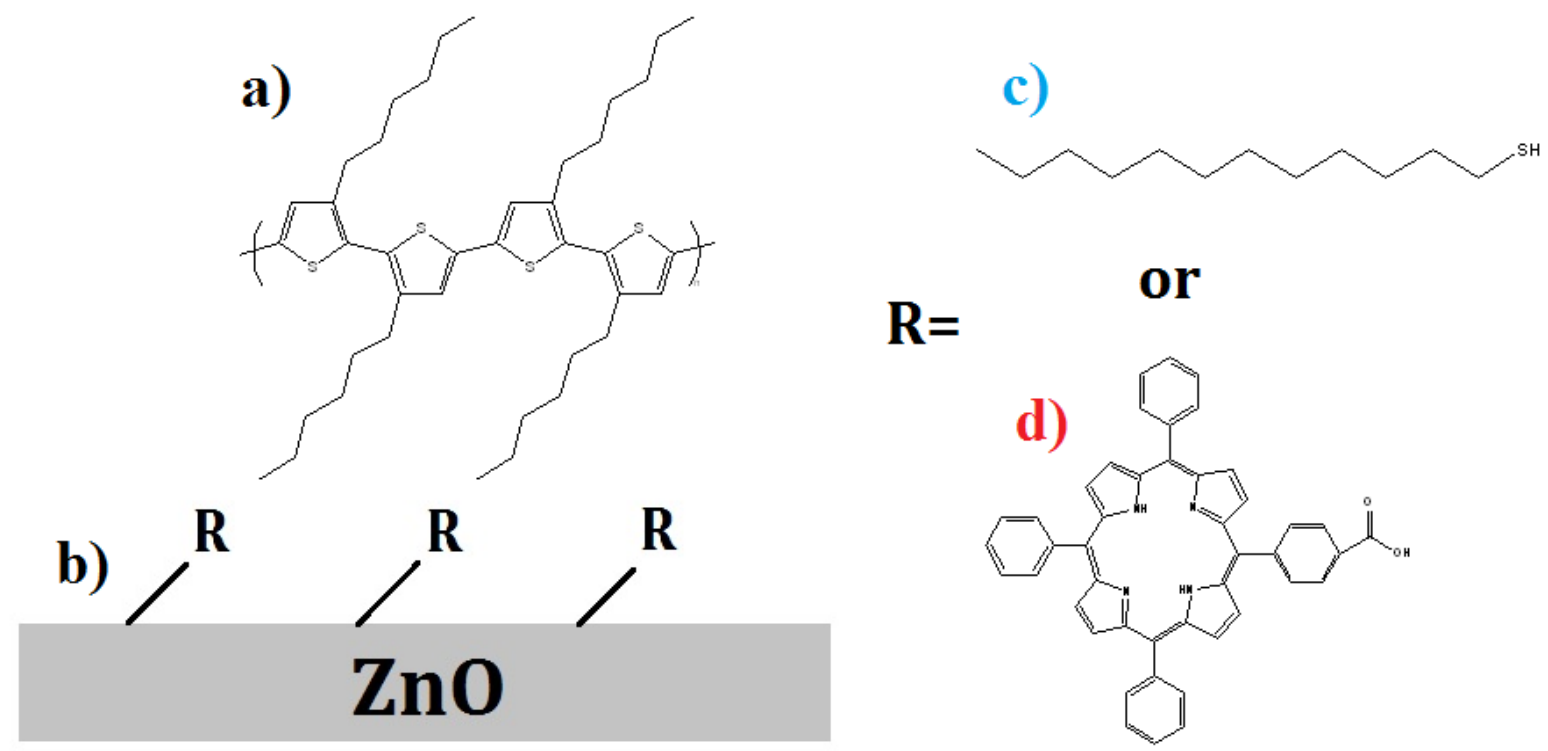

Figure 2.2. Scheme of chemical structures used for modification of $\mathrm{ZnO}$ and $\mathrm{P} 3 \mathrm{HT}$ kinetics. (a) P3HT, (b) Modified $\mathrm{ZnO}$ with $\mathrm{R}$ representing one of two ligands, (c) DDT ligand, and (d) TPP ligand.

\subsubsection{Quantum Dot Attachment to $\mathrm{ZnO}$ Nanowire}

Commercial CdSe quantum dots (gradient alloyed $\mathrm{ZnS}$ coated, Mesolight LLC), TOPO-CdSe quantum dots (provided by Dr. Richard Savage of the Materials Engineering Department, California Polytechnic State University San Luis Obispo), 
3-Aminopropyltrimethoxysilane (APS), 3-Mercaptopropyltrimethoxysilane (MPS), were used without further purification. The $\mathrm{ZnO}$ white powder was dried in an oven at $60^{\circ} \mathrm{C}$ and pressed into a finer powder using a spatula. Batches of APS-modified $\mathrm{ZnO}$ and MPSmodified $\mathrm{ZnO}$ were prepared by adding $5 \mathrm{mg} \mathrm{ZnO,} 3 \mathrm{~mL}$ DMSO, and $5 \mu \mathrm{L}$ of APS ligand or MPS ligand. Initial batches were heated to $130^{\circ} \mathrm{C}$ and stirred for 5 hours to be consistent with literature, but subsequent batches using vortexing at room temperature for 24 hours showed no difference, so the latter procedure was used. The modified $\mathrm{ZnO}$ was then rinsed three times with EtOH to remove excess ligand. Samples were then redispersed in toluene and sonicated for 30 minutes. Quantum dots were then added to the mixture and samples were vortexed for three days at room temperature. Samples were rinsed with toluene three times to remove excess quantum dots.

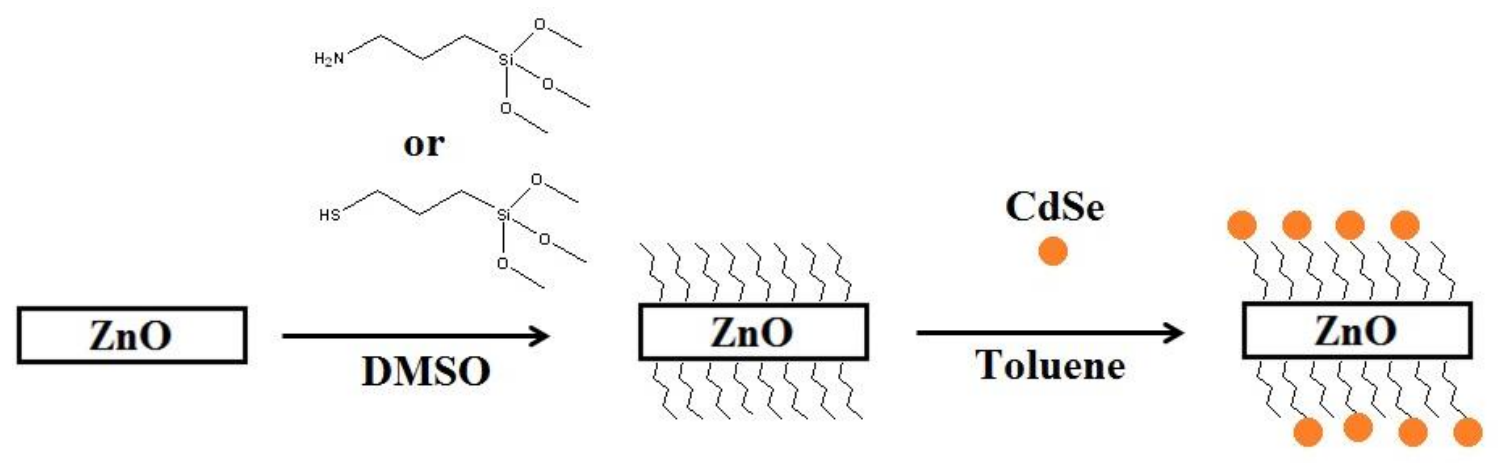

Figure 2.3. Reaction scheme and chemicals used in surface modification of $\mathrm{ZnO}$ nanowire with quantum dots.

\subsection{Characterization and Analysis}

\subsubsection{Fourier Transform Infrared Spectroscopy}

Fourier transform infrared (FTIR) spectra were obtained using a Nicolet 380 FTIR spectrometer in attenuated total reflection (ATR) mode at a resolution of $4 \mathrm{~cm}^{-1}(1000$ 
scans). IR spectra were obtained to characterize the modification of $\mathrm{ZnO}$ through a shift in ligand side group vibration. Spectra of liquid samples including DDT, MPS, and APS were obtained in the liquid phase. Spectra of solid samples including P3HT, TPP, ZnO, modified $\mathrm{ZnO}$, and $\mathrm{CdSe}$ quantum dots were obtained by creating a thin film on glass slides by dissolving or dispersing in chloroform and evaporating at room temperature.

\subsubsection{UV-visible Spectroscopy}

UV-visible spectra were taken on a Jasco V-550 Uv-visible spectrometer with glass cuvettes sealed from ambient atmosphere. Temperature sensitive experiments employed the use of a Peltier Thermostat. UV-visible spectra were obtained either for characterization of TPP-modified ZnO or P3HT crystallization kinetics. P3HT samples were prepared via a stock solution of $2.00 \mathrm{mg}$ P3HT and $10.00 \mathrm{~mL}$ anisole in a round bottom flask to produce a $0.2 \mathrm{mg} \mathrm{mL}^{-1}$ or $3.4 \times 10^{-6} \mathrm{M}$ stock solution. The solution was first heated to $80^{\circ} \mathrm{C}$ for 30 minutes to dissolve the polymer. For temperature dependent isotherms, the internal temperature of the spectrometer was decreased by a set amount and the solution was monitored by observing the absorbance change at a $\lambda_{\max }$ of $600 \mathrm{~nm}$. Once the absorbance change had stabilized to a flat line for 5 minutes, the individual UVvisible spectrum was obtained. The temperature was then lowered again and this procedure was repeated to obtain UV-visible spectra of the sample across the desired temperature range. 


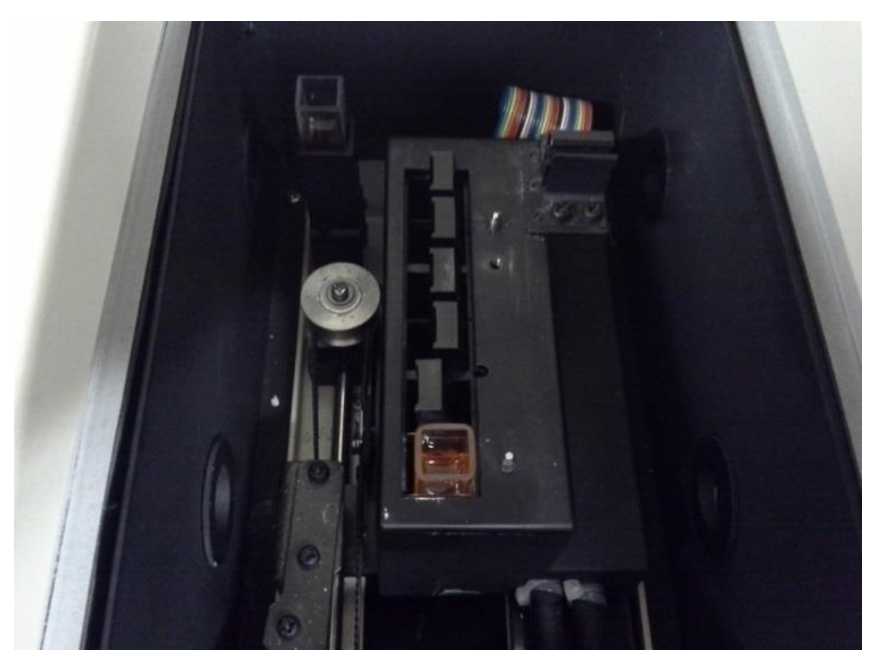

Figure 2.4. Inside of the Jasco UV-visible spectrometer used in this work with blank cuvette in the upper left and cuvette with P3HT solution at bottom.

Heating and cooling curves were obtained by equilibrating a $0.85 \mu \mathrm{M}$ P3HT solution for at least 30 minutes at the upper or lower temperature, and measuring a $\lambda_{\max }$ of $600 \mathrm{~nm}$. Curves were obtained during a 30 minute interval, corresponding to a temperature ramp of $2^{\circ} \mathrm{C} \min ^{-1}$. Heating curves for the determination of $\mathrm{T}_{\mathrm{d}}{ }^{0}$ were obtained in a similar fashion, but used a temperature ramp of $1^{\circ} \mathrm{C} \min ^{-1}$. Heating curves were obtained across a crystallization temperature range from $15^{\circ} \mathrm{C}$ to $30^{\circ} \mathrm{C}$. The $\mathrm{T}_{\mathrm{d}}$ values were estimated by smoothing the heating curve data and obtaining the inflection point where the second derivative was equal to 0 . Time dependent isotherms were obtained by equilibrating an initial sample of $0.68 \mu \mathrm{M}$ P3HT for 30 minutes at $80^{\circ} \mathrm{C}$. The sample was then placed in the UV-visible spectrometer with an internal temperature of $25^{\circ} \mathrm{C}$ and monitored over time at a $\lambda_{\max }=600 \mathrm{~nm}$. Individual kinetics runs were produced by first allowing the $\mathrm{P} 3 \mathrm{HT}$ solution to equilibrate at $80^{\circ} \mathrm{C}$ for at least 5 minutes and then placing the sample in the spectrometer with the desired internal spectrometer temperature 
(data is shown for a $\mathrm{T}_{\mathrm{c}}$ of $35^{\circ} \mathrm{C}$ ). The samples were monitored at a $\lambda_{\max }$ of $600 \mathrm{~nm}$ for 2500 seconds. Initial rates from these runs were determined by obtaining the slope of the best fit line for the first 200 seconds of the experiment. Reaction order was determined by the slope of the best fit line of the $\log$ (initial rate) vs. $\log$ (concentration) plot. Quantum dot characterization was carried out by diluting a $1 \mu \mathrm{L}$ solution of CdSe quantum dots with $3 \mathrm{~mL}$ of toluene. Spectra were obtained with an internal spectrometer temperature of $20^{\circ} \mathrm{C}$ and $50^{\circ} \mathrm{C}$.

\subsubsection{Fluorescence Spectroscopy}

Photoluminescence emission spectra were acquired using a Jasco SP-6500 fluorescence spectrometer. All samples were measured in an unfrosted quartz cuvette and sealed from atmospheric conditions. An excitation wavelength of $490 \mathrm{~nm}$ was chosen to observe both P3HT and quantum dots (PL=540 nm). P3HT fluorescence was measured with the addition of modified $\mathrm{ZnO}$ to determine possible quenching activity of the physically attached nanowire. A $3.4 \times 10^{-6} \mathrm{M}$ P3HT stock solution was diluted in a cuvette until the maximum intensity of the fluorescence spectrum decreased below 500. Modified $\mathrm{ZnO}$ was added prior to the dilution at a loading of $10 \%\left(0.002 \mathrm{mg} \mathrm{mL}^{-1}\right)$ and $100 \%(0.02$ $\mathrm{mg} \mathrm{mL} \mathrm{m}^{-1}$ ). Fluorescence spectra for quantum dots were also taken to characterize them and to determine possible quenching activity from nearby P3HT. Characterization spectra were taken at volumes of $1 \mu \mathrm{L}$ to $10 \mu \mathrm{L}$ CdSe quantum dots in toluene. The concentration of the quantum dots after synthesis was not acquired, so data was obtained in terms of relative volume of the original stock solution of quantum dots. 
3. Results and Discussion

\subsection{Interaction of Modified $\mathrm{ZnO}$ and P3HT}

\subsubsection{Characterization of Modified $\mathrm{ZnO}$}

The preparation of TPP-modified $\mathrm{ZnO}$ produced a distinct color change from the white $\mathrm{ZnO}$ powder and pink TPP ligand to form a brown solid. DDT-modified ZnO showed no change from the white $\mathrm{ZnO}$ powder and clear DDT ligand when modified. The chemical attachment of the DDT ligand to $\mathrm{ZnO}$ was verified through IR spectroscopy, as seen in Figure 3.1. ZnO nanowire displays very few vibrational modes if any in the infrared region. Doublet peaks seen around $2300 \mathrm{~cm}^{-1}$ are due to $\mathrm{CO}_{2}$ interference. Literature focused on the chemical attachment of thiol ligands to inorganic material suggests that there is a shift in the triplet $\mathrm{C}-\mathrm{H}$ stretching seen around $1900 \mathrm{~cm}^{-}$ ${ }^{1}{ }^{[19]}$ More specifically, the locations of the methylene $\mathrm{C}-\mathrm{H}$ stretch modes provide information of the conformation of the alkyl chains. Tightly packed alkyl chains from the DDT ligands will exhibit a shift of about $5 \mathrm{~cm}^{-1}$ in both the symmetric and asymmetric methylene stretch modes around $2850 \mathrm{~cm}^{-1}$ and $2915 \mathrm{~cm}^{-1}$. These shifts are sometimes difficult to substantiate and without well-defined, tightly packed ligands and a highresolution spectrum cannot be detected. The modified DDT- $\mathrm{ZnO}$ displayed the methylene stretches confirming the presence of the DDT ligand, but did not show any characteristic peak shifts in this region. Another possible method of detecting the thiol attachment is the direct shift of an S-H stretching from the DDT-ligand to an $\mathrm{SO}_{2}$ band. ${ }^{[23]}$ This can however be difficult, as the thiol stretching around $2550-2600 \mathrm{~cm}^{-1}$ has very low intensity. These peaks were not observed in the DDT ligand or DDT-modified ZnO. 


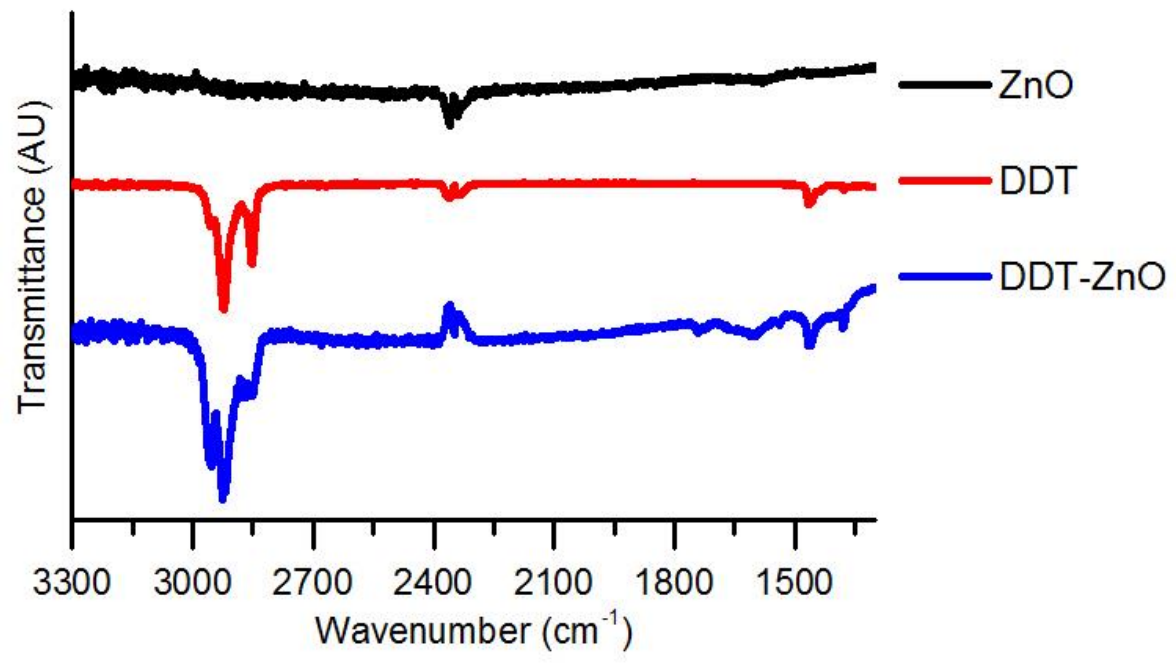

Figure 3.1. IR spectra of $\mathrm{ZnO}$, DDT, and DDT-modified ZnO.

The attachment of the TPP ligand to $\mathrm{ZnO}$ was also investigated using IR spectroscopy, seen in Figure 3.2. The carboxylic acid attachment provides a more direct method in measuring the attachment. The $-\mathrm{COOH}$ side group participates in hydrogen bonding and the $\mathrm{C}=\mathrm{O}$ carbonyl displays a sharp stretching band around $1700 \mathrm{~cm}^{-1}$. Once this group has linked to the nanowire it is believed to form a COOZn bond, where the ligand attaches in a bidentate fashion. This linkage removes the possibility for hydrogen bonding and also shifts the distinct $\mathrm{C}=\mathrm{O}$ band to a $-\mathrm{CO}_{2}$ band around $1560 \mathrm{~cm}^{-1} \cdot{ }^{[23,32]}$ As seen in Figure 3.2, the pure TPP ligand clearly shows a carbonyl stretching, but when it is chemically attached to the nanowire, this peak disappears. It is believed that the $\mathrm{C}=\mathrm{O}$ stretch may be shifted into the already prominent $1540 \mathrm{~cm}^{-1}$ peak seen in TPP alone. Regardless, the IR spectra confirm the disappearance of free carboxylic acid group after chemical attachment to the nanowire. 


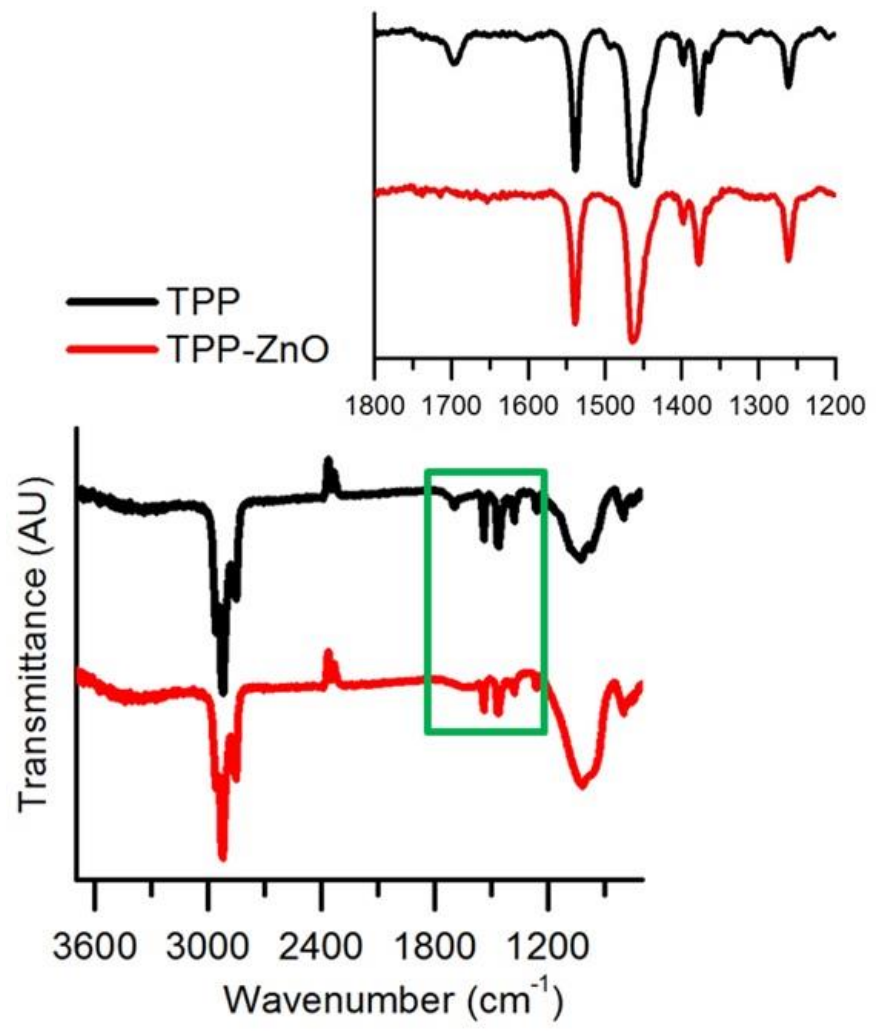

Figure 3.2. IR spectra of TPP and TPP-modified $\mathrm{ZnO}$, with inset of zoomed in region from $1200-1800 \mathrm{~cm}^{-1}$ to highlight the disappearance of carbonyl stretching after modification.

UV-visible spectroscopy was also employed to further substantiate the chemical attachment of the DDT and TPP ligands (Figure 3.3). While IR spectroscopy is useful in understanding the change in chemical bond vibrations, UV-visible spectroscopy can reveal any physical effects that the modification procedure has on the conformation of nanowire or ligand. $\mathrm{ZnO}$ nanowire displays a characteristic broad absorbance spectrum peaking around $370 \mathrm{~nm}$, which corresponds to a band gap energy of $3.37 \mathrm{eV}$. Not shown in Figure 3.3 is the UV-visible spectrum of the DDT ligand, as the ligand appears clear and does not absorb light in this region, and thus displays no features. It follows that the $\mathrm{UV}$-visible spectrum of DDT-modified $\mathrm{ZnO}$ then appears identical to pure $\mathrm{ZnO}$, 
demonstrating that the attachment does not change the electronic properties of the nanowire. The pink TPP ligand however shows strong absorbance in the visible region, and because of its conjugated ring system contains many characteristic peaks. The most prominent peak in this region is referred to as the Soret band at $411 \mathrm{~nm}$, followed by peaks around 509, 543, 587, and $646 \mathrm{~nm}$. The inset of Figure 3.3 emphasizes the shifts in these characteristic peaks after the porphyrin ring has been attached to the $\mathrm{ZnO}$ nanowire. The Soret band undergoes an $11 \mathrm{~nm}$ red shift, suggesting that the porphyrin center of the TPP ligand has undergone a conformational shift. This combined with the obvious change in the ligand's spectrum from 500 to $650 \mathrm{~nm}$ suggests that the attached TPP is interacting with neighboring ligands through covalent bonding, altering its electronic properties. ${ }^{[68]}$ It has been suggested that the large porphyrin rings stack with themselves perpendicular to the nanowire surface through pi-pi stacking. ${ }^{[36]}$ It should also be noted that the $370 \mathrm{~nm}$ peak due to $\mathrm{ZnO}$ seen in the TPP-modified $\mathrm{ZnO} \mathrm{UV}$-visible spectrum has not shifted, implies the electronic structure of the nanowire is unchanged as expected. 


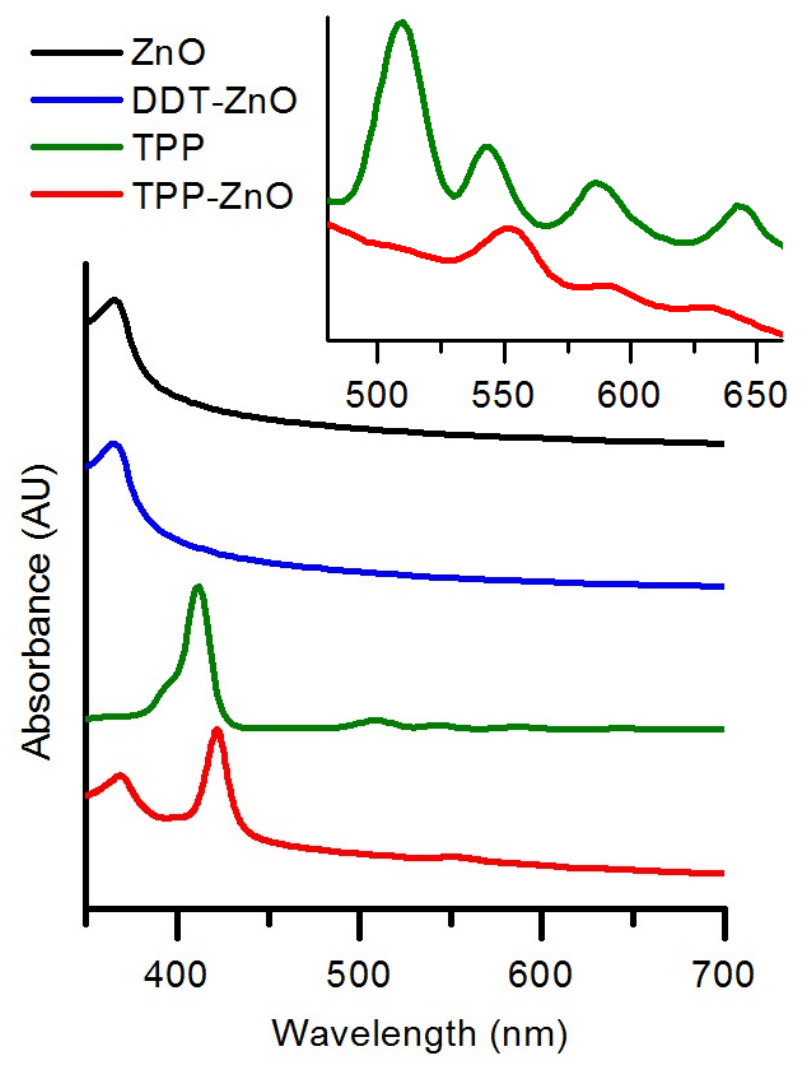

Figure 3.3. UV-visible spectra of ZnO, DDT-modified ZnO, TPP, and TPP-modified $\mathrm{ZnO}$. Inset is a zoomed in comparison of TPP and TPP-modified $\mathrm{ZnO}$ to highlight the shift in characteristic porphyrin peaks.

\subsubsection{Effect of Modified Nanowire on P3HT Optical Properties}

As stated previously, fluorescence spectroscopy can be a valuable tool in determining if there are electron transfer properties between two materials. Figure 3.4 shows the fluorescence spectrum of P3HT at an excitation wavelength of $490 \mathrm{~nm}$, along with the spectra of $\mathrm{P} 3 \mathrm{HT}$ with added modified $\mathrm{ZnO}$. It is apparent that the fluorescence profile does not change with addition of significant amounts of modified $\mathrm{ZnO}(10 \%$ loading is typical in hybrid devices). This lack of quenching suggests there is no path of charge transport from P3HT to the nanowire. This could either mean the nanowire is 
insufficiently surface modified so that there is little to no surface interaction between nanowire and polymer, or there is interaction, but electrons are unable to transfer. Previous experiments have shown extreme quenching of $\mathrm{P} 3 \mathrm{HT}$ with $\mathrm{ZnO} / \mathrm{P} 3 \mathrm{HT}$ nanocomposites, confirming that there is efficient electron injection when the materials are in close contact. ${ }^{[18]}$ It is possible that the ligands attached to $\mathrm{ZnO}$ are bulky enough that they limit electron transfer from polymer to nanowire, however previous experiments have also shown that both aliphatic and aromatic ligands of this size produce functional hybrid solar cells with efficiencies higher than those without ligands. ${ }^{[33,69]}$ Whatever the cause, the lack of quenching does not indicate there is no interaction between the nanowire and polymer. The modified nanowire could also potentially benefit the physical conformation of the polymer and improve miscibility.

(a)

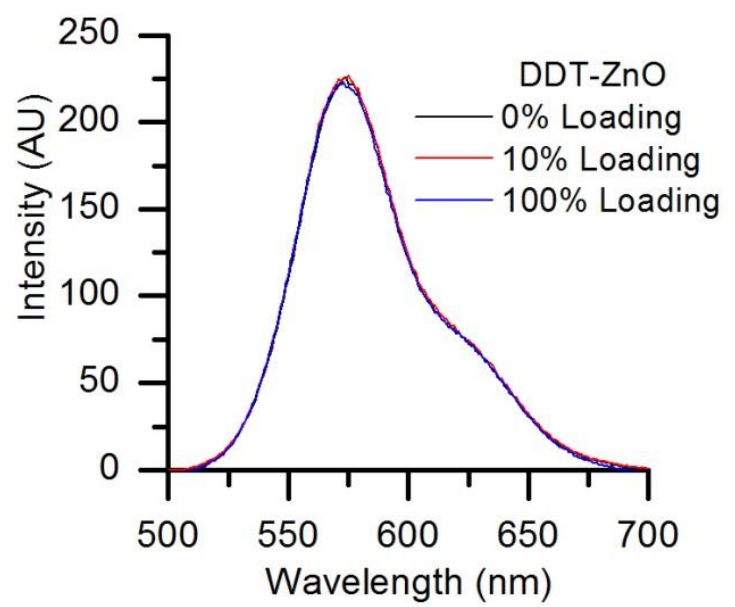

(b)

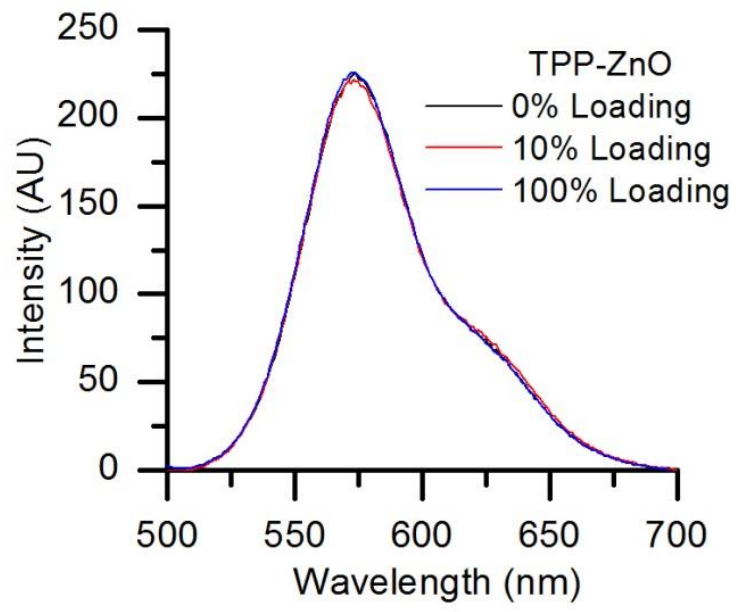

Figure 3.4. Addition of modified $\mathrm{ZnO}$ showing a lack of effect on the fluorescence spectra of P3HT. 
To observe the effect of nanowire on the physical properties of P3HT, UV-visible spectroscopy was used to observe changes in the polymer absorbance spectrum. Figure 3.5 (a) displays the shift in the UV-visible absorbance of P3HT at different temperatures in a poor solvent. At a higher temperature, the polymer is fully solvated in a coil-like conformation. As the polymer solution cools, the single chains transition into a rod-like state, which also brings about individual chain crystallization and aggregation. The combination of these effects results in a decrease in the amplitude of the $450 \mathrm{~nm}$ peak attributed to coil-polymer, and an increase in amplitude of the shoulders around $560 \mathrm{~nm}$ and $600 \mathrm{~nm}$ due to crystallized polymer. It should also be noted that the $450 \mathrm{~nm}$ peak undergoes a red shift as polymer crystallizes at lower temperatures, possibly due to an increase in the polymer's effective conjugation length.

(a)

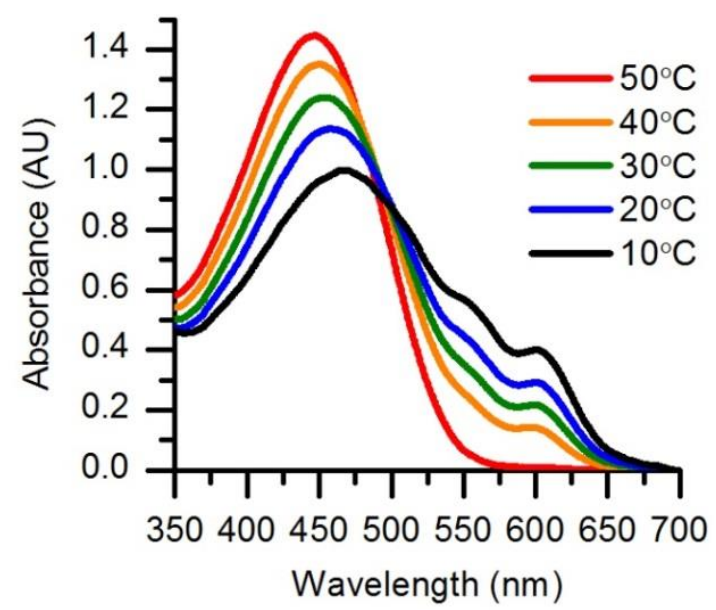

(b)

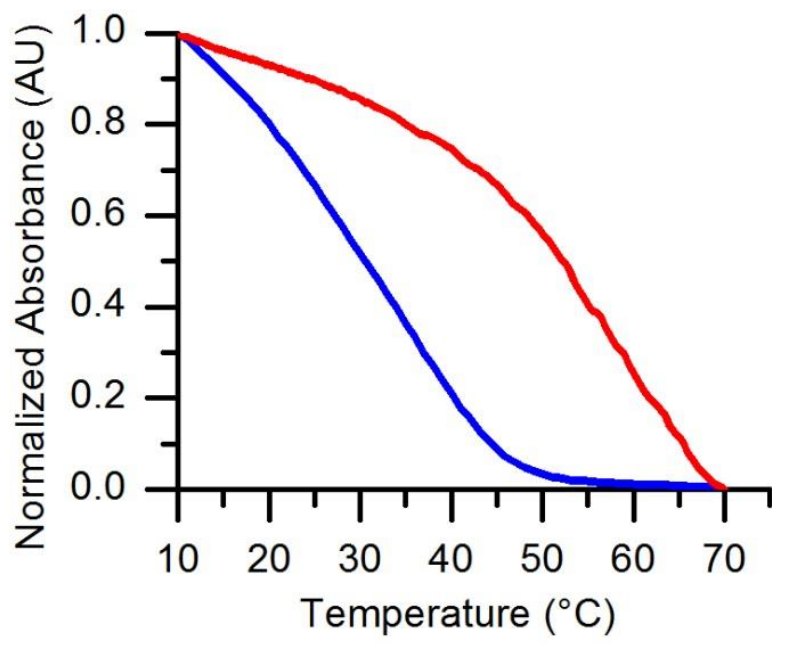

Figure 3.5. P3HT thermochromism displayed via UV-visible spectroscopy. (a) The temperature dependence of $\mathrm{P} 3 \mathrm{HT}$ conformation, (b) Heating and cooling curves of P3HT taken at a $\lambda$ of $600 \mathrm{~nm}$ in anisole. 
This peak can then be measured across a large temperature range to produce a temperature curve that reveals how much polymer is crystallized at any given temperature. These temperature curves can either be taken by heating the polymer from a fully crystallized state, or cooling it from a fully amorphous state (Figure 3.5). At an infinitely slow cooling/heating rate these two curves would look identical, but a finite temperature ramp will produce a "delay" in the amount of crystallized polymer, called hysteresis. The temperature curves in Figure 3.5 (b) display broad hysteresis, signifying that the crystallization range or dissolution range will be largely temperature dependent. Because the crystallization and dissolution process is due to polymer-solvent interactions, these temperature curves are also strongly solvent dependent. The amount of hysteresis and the range of the transition temperature will differ from solvent to solvent. ${ }^{[70]}$ These curves are also used to determine critical transition points. The dissolution temperature $T_{d}$ is defined as the inflection point along the heating curve, and in the case of P3HT in anisole lies around $60^{\circ} \mathrm{C}$. The crystallization temperature $\mathrm{T}_{\mathrm{c}}$ is defined as the inflection point along the cooling curve, and in the case of P3HT in anisole lies around $30^{\circ} \mathrm{C}$.

Figure 3.6 displays the four original cooling curves acquired incorrectly. The data for this figure was acquired sequentially from a heated stock solution. The first cooling curve obtained for P3HT looks fairly regular, while the P3HT with DDT-ZnO solution seems to have a lower absorbance and some slight irregularities towards lower temperatures. This trend continues with P3HT and TPP-ZnO and finally with P3HT and $\mathrm{ZnO}$, the curve has a very low absorbance and a jagged appearance at lower temperatures. The final cooling curves were taken from a stock solution of P3HT that had been continuously heated the whole time. It is believed that over long periods of 
continuous heating, the polymer chains will begin to oxidize. What this means in terms of Figure 3.6 is that the cooling curves taken later exhibit oxidize P3HT chains and a much larger affinity to aggregate with neighboring chains. Thus, the jagged appearance of the curves at lower temperatures and lower absorbance signifies oxidized P3HT chains and not any type of interaction from the nanowire. It is important to understand that polymer crystallization can be affected by a large number of factors and early conclusions in apparent trends can be grossly incorrect.

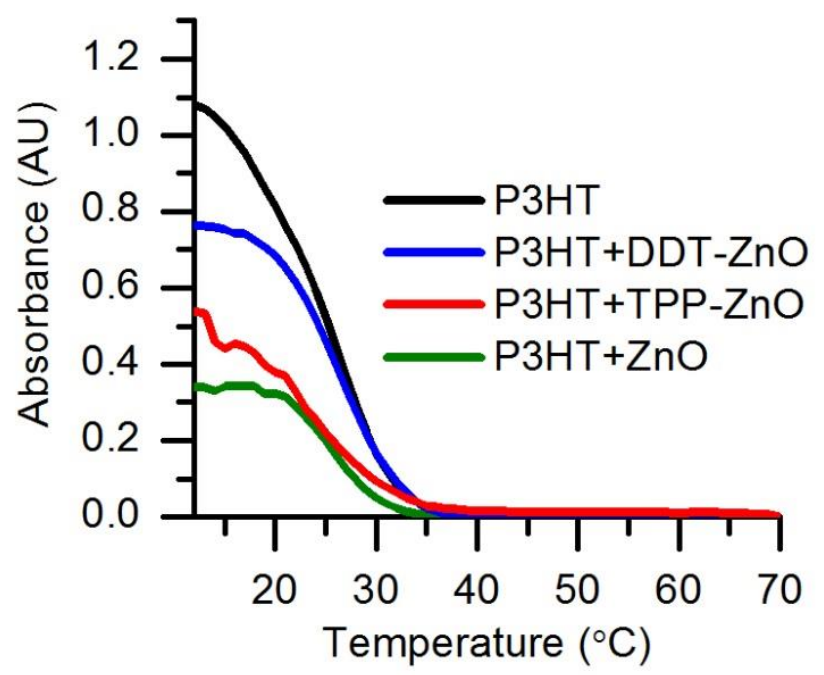

Figure 3.6. Cooling curves for P3HT, P3HT with DDT-modified ZnO, P3HT with TPPmodified $\mathrm{ZnO}$, and $\mathrm{P} 3 \mathrm{HT}$ with $\mathrm{ZnO}$. Data reflects poor experimental procedure and displays the tendency for aggregate polymer formation over time.

Figure 3.7 on the other hand displays the cooling curves and heating curves of P3HT with and without nanowire with un-oxidized P3HT. Here it is obvious that the modified nanowire has little if any effect on the amount of polymer crystals at any given temperature. The curves were analyzed for any inherent trends in $T_{c}$ or $T_{d}$, but no evidence was found to support the claim that modified nanowire affects the polymer crystallization significantly. 


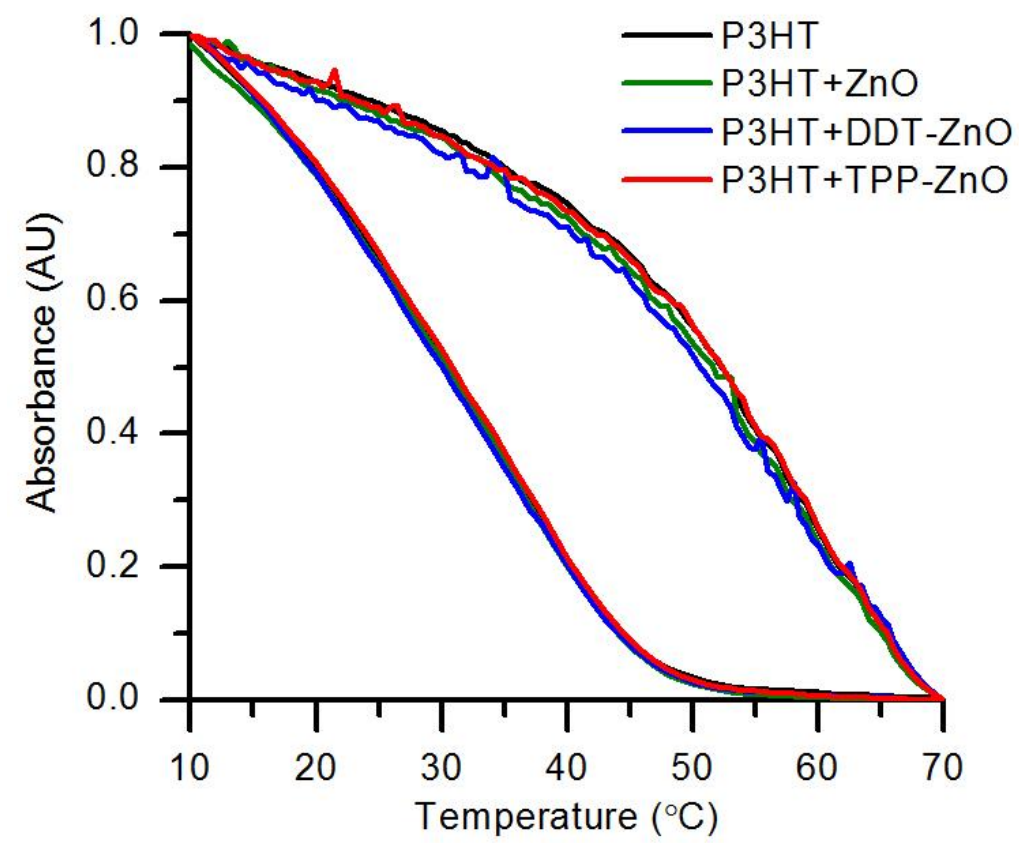

Figure 3.7. Correctly acquired cooling and heating curves of the four systems.

\subsubsection{Kinetics of P3HT Crystallization}

The preliminary experiments show that the modified $\mathrm{ZnO}$ has little effect on the crystallization behavior of P3HT. However, the results do not shed light on the mechanism of $\mathrm{P} 3 \mathrm{HT}$ crystallization, or if nanoparticles can potentially affect this reaction mechanism, possibly through providing additional nucleation sites. To quantify this effect, the kinetics of P3HT crystallization is observed through time dependence as opposed to temperature dependence. Figure 3.8 (a) shows this time dependent crystallization as a solution of $\mathrm{P} 3 \mathrm{HT}$ is cooled from a high temperature to below its $\mathrm{T}_{\mathrm{c}}$ and is monitored. The UV-visible spectra show the same features as the temperature dependent isotherms, so the rate of crystallization can then be measured as a function of the Absorbance at $600 \mathrm{~nm}$ against time, seen in Figure 3.8 (b). 
(a)

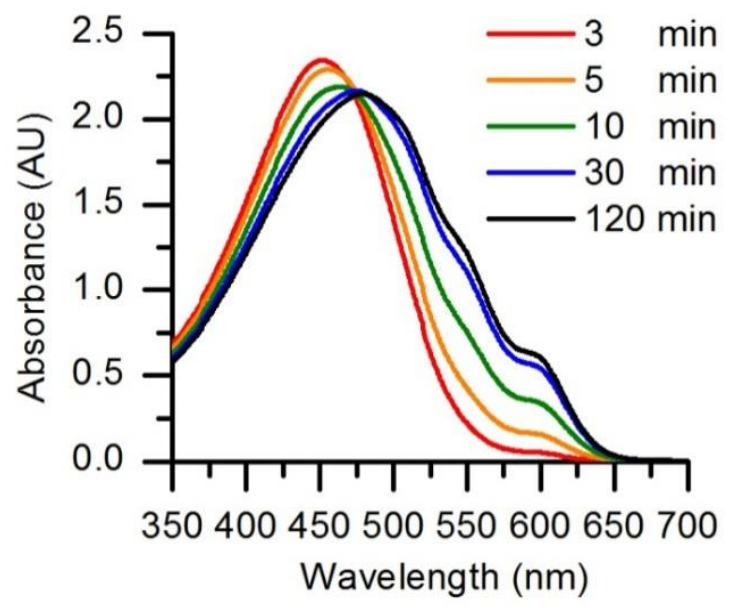

(b)

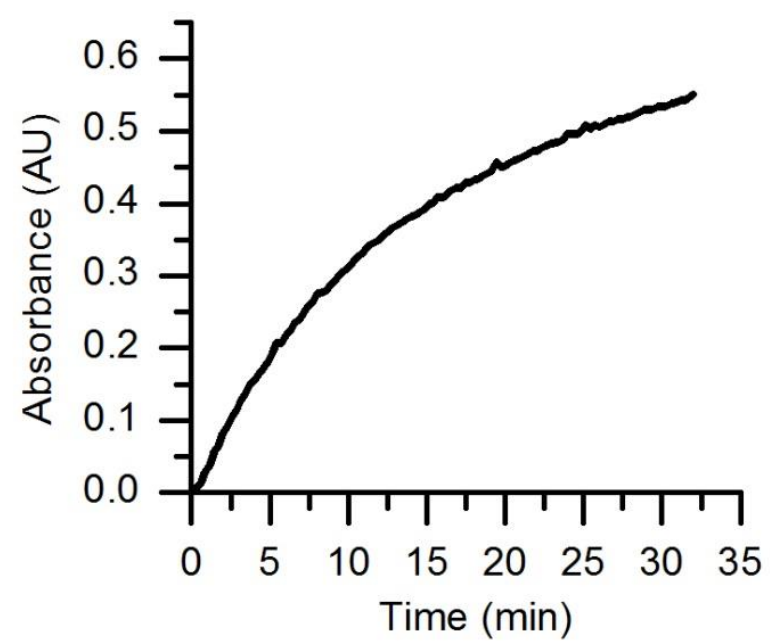

Figure 3.8. Kinetics of P3HT conformational change. (a) Isotherms showing the time dependence of P3HT crystallization, (b) Single sample P3HT crystallization kinetics, taken at a $\lambda$ of $600 \mathrm{~nm}$.

This time dependent crystallization was measured at several different $\mathrm{T}_{\mathrm{c}}$ 's, shown in Figure 3.9. Obviously, the shape of the kinetics curves depends on the crystallization temperature $\mathrm{T}_{\mathrm{c}}$. It also seems that at lower temperatures $\left(\sim 20^{\circ} \mathrm{C}\right)$ the maximum rate of crystallization does not occur until several minutes into the cooling process. This creates more of a challenge when deciding the initial rate of polymer crystallization at low undercooling temperatures. The initial rate of $\mathrm{P} 3 \mathrm{HT}$ kinetics were taken at crystallization temperatures at or above $25^{\circ} \mathrm{C}$ for accuracy in measuring the initial rate. 


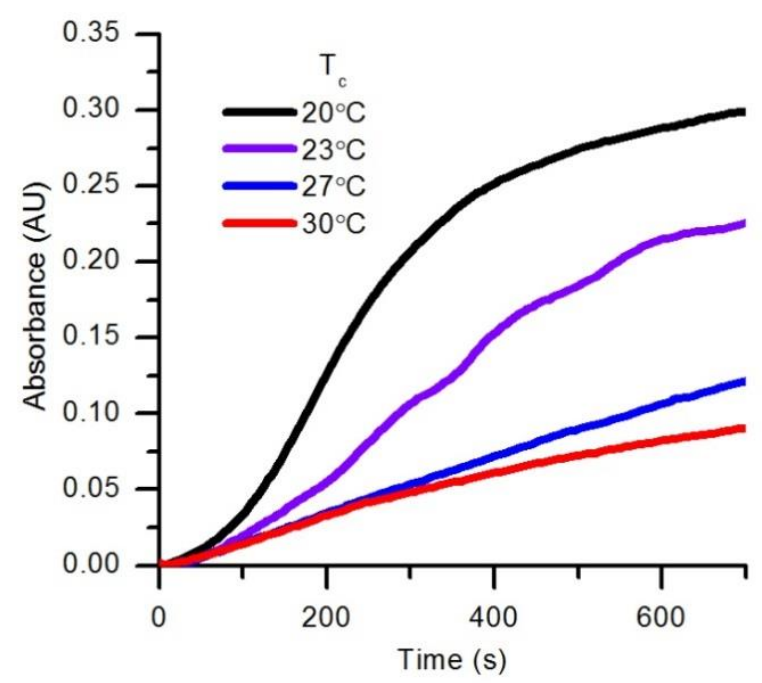

Figure 3.9. Kinetics of P3HT crystallization at different crystallization temperatures $\mathrm{T}_{\mathrm{c}}$, measured by monitoring the $600 \mathrm{~nm}$ peak over time via UV-visible spectroscopy.

As stated previously, reaction rate equations can be plotted by utilizing the method of initial rates by measuring the initial rate of a transition as a function of the concentration of reactant. In this case we can measure the in-situ crystallization rate of polymer by monitoring the initial rate at different concentrations for all four P3HT systems (Figure 3.10). Here the P3HT and modified-ZnO P3HT kinetics are measured at a $\mathrm{T}_{\mathrm{c}}$ of $35^{\circ} \mathrm{C}$, leading to initial rates that appear somewhat linear. The P3HT with unmodified $\mathrm{ZnO}$ (Figure 3.10 (b)) was actually measured at a $\mathrm{T}_{\mathrm{c}}$ of $25^{\circ} \mathrm{C}$ leading to a drastically different appearance, and a plateau is reached fairly quickly. This mistake means that the rate constants " $\mathrm{k}$ " determined for $\mathrm{P} 3 \mathrm{HT}$ with $\mathrm{ZnO}$ will not be comparable to the other three rate constants, because of the highly temperature dependent nature of the constant. This does still however allow for similar treatment of the data in terms of reaction order " $\alpha$ ", as the reaction order is dependent on the mechanism of crystallization. Fortunately, the reaction order is the most important piece of information gathered from this reaction rate analysis, and a change in experimental parameters will not affect the end result. 
(a)

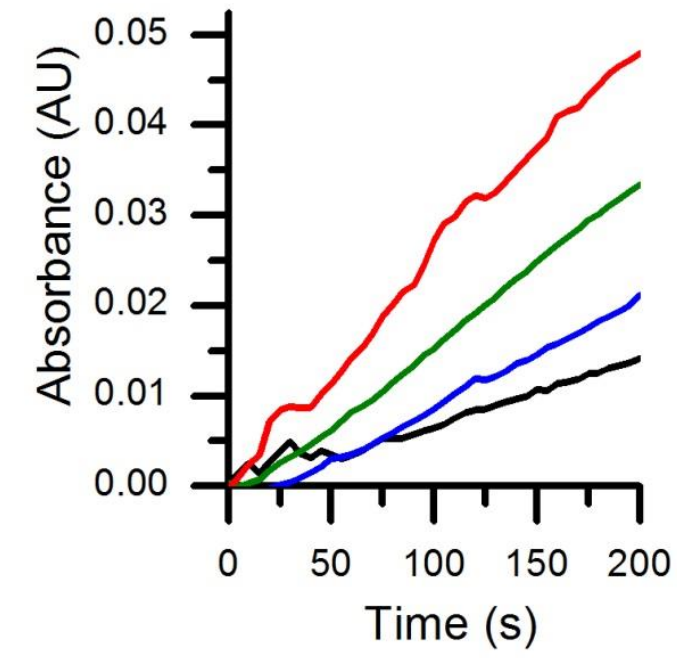

$-0.45 \mu \mathrm{M}$

(c)

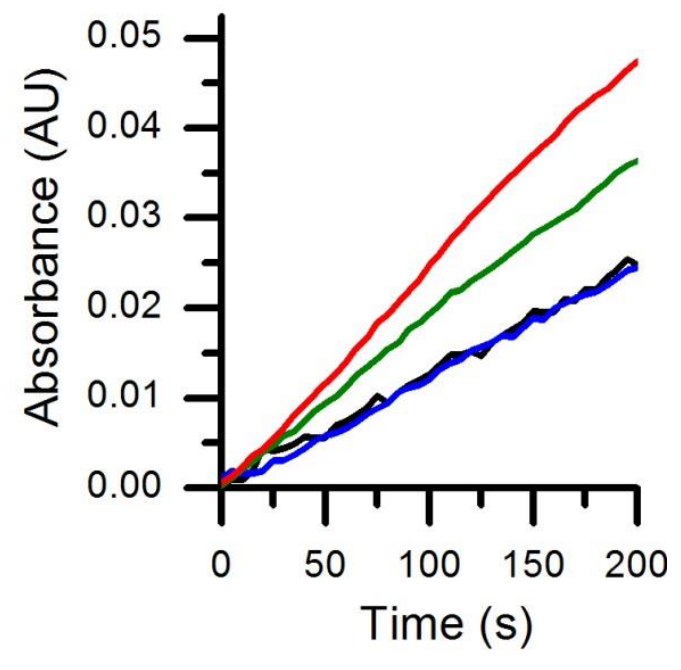

(b)

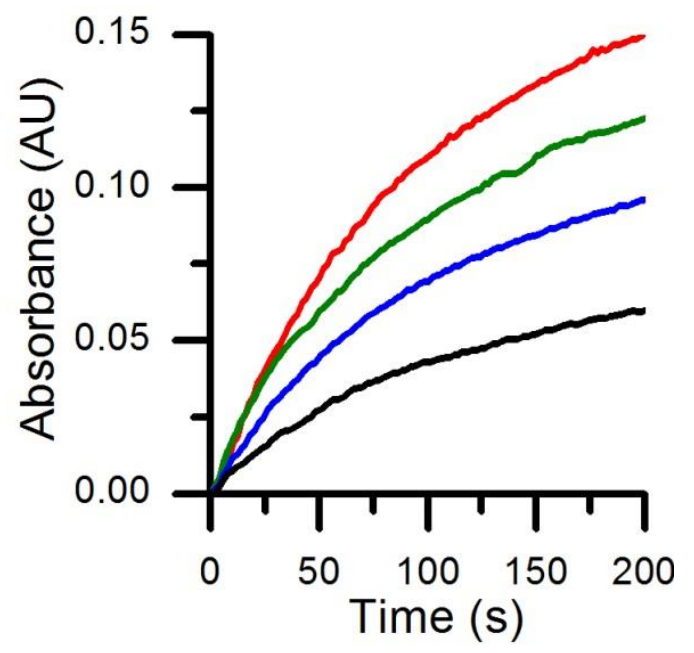

$0.30 \mu \mathrm{M}$

(d)

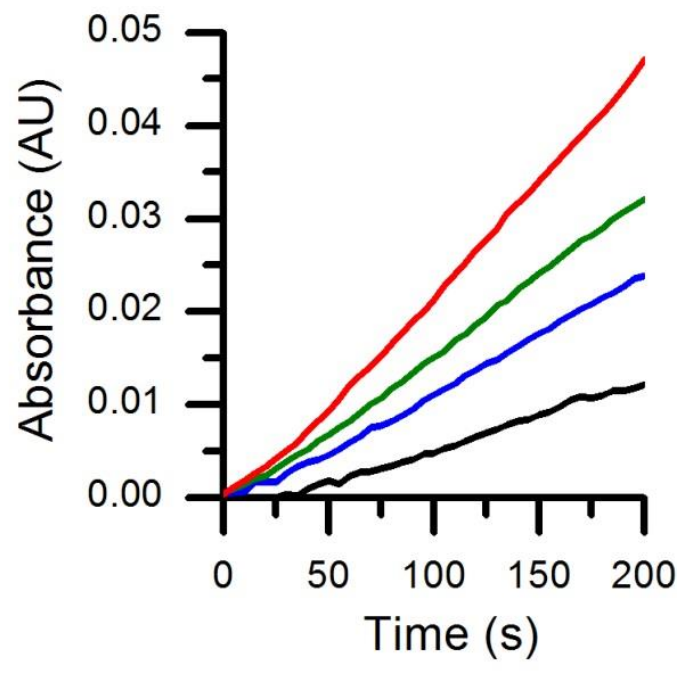

Figure 3.10. Concentration dependence of P3HT crystallization for (a) P3HT, (b) P3HT with $\mathrm{ZnO}$, (c) P3HT with DDT-modified $\mathrm{ZnO}$, and (d) P3HT with TPP-modified ZnO. The kinetics of $\mathrm{P} 3 \mathrm{HT}$ with $\mathrm{ZnO}$ in (b) were taken at a different $\mathrm{T}_{\mathrm{c}}$, leading to a drastically different appearance than the other three systems. 
Figure 3.11 shows the experimental data from Figure 3.10 plotted as a function of initial crystallization rate against concentration as outlined in Equation 1.4. The use of different $\mathrm{T}_{\mathrm{c}}$ in the $\mathrm{P} 3 \mathrm{HT}$ with $\mathrm{ZnO}$ sample has a drastic effect on the slope and position of the data points. The data seems to fit a linear trend, and in the case of first order kinetics the slope of the best fit line in these plots is then the rate constant k. So it follows that when crystallized at a lower temperature, the rate constant is much higher as is the case in the P3HT with $\mathrm{ZnO}$ sample. Figure 3.11 (b) shows the same data with the P3HT with $\mathrm{ZnO}$ removed to compare the remaining samples. It is clear the reaction rate kinetics for these three samples are extremely similar.
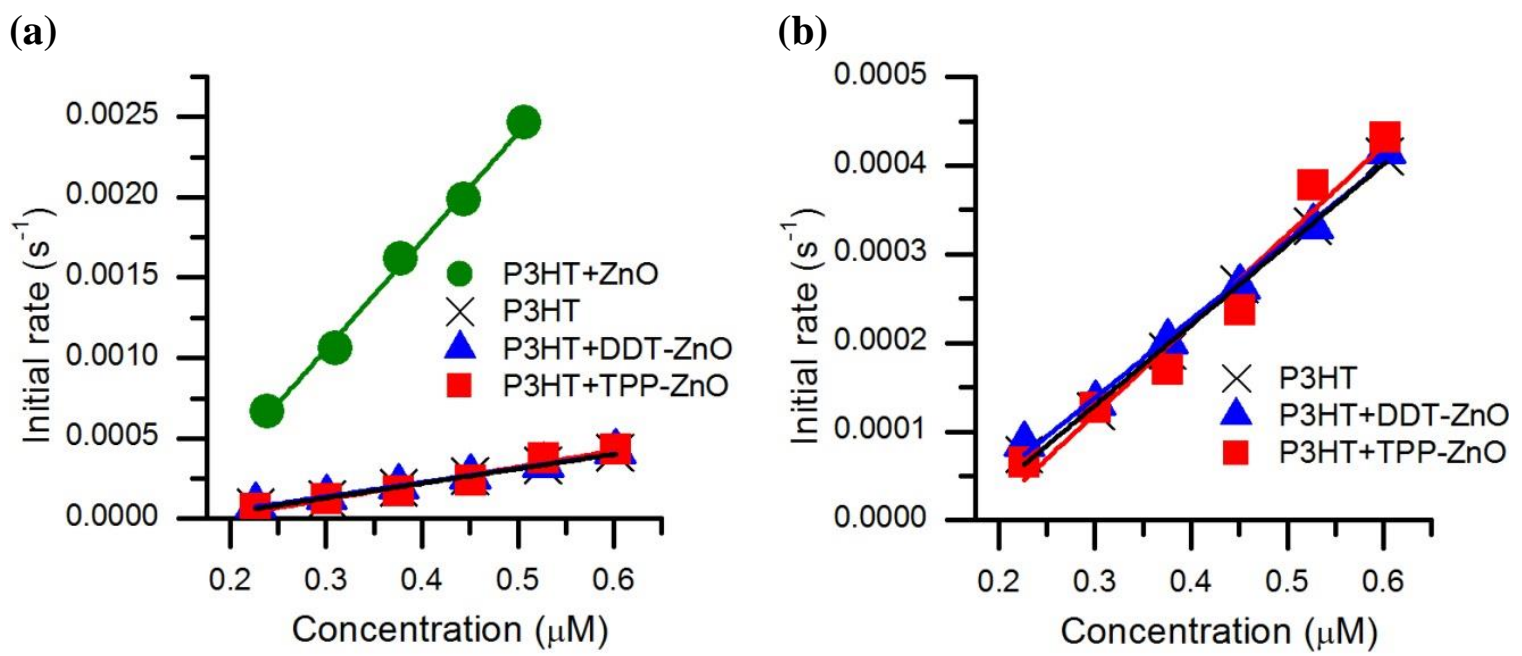

Figure 3.11. Experimental reaction rate data gathered via method of initial rates for P3HT, P3HT with ZnO, P3HT with DDT-modified ZnO, and P3HT with TPP-modified $\mathrm{ZnO}$. All four systems are shown in (a), while the P3HT with $\mathrm{ZnO}$ kinetics (done at a different $T_{c}$ ) were removed in (b) for clarity. 
Figure 3.12 shows the experimental data plotted as the $\log$ (initial rate) against the $\log ($ concentration $)$ as detailed in Equation 1.5. The data closely resembles that of Figure 3.11, however the slope of the best fit lines here represent the reaction order of the crystallization process, which should theoretically be independent of $\mathrm{T}_{\mathrm{c}}$. The P3HT with $\mathrm{ZnO}$ sample is still significantly different than the other three, but the slope of the best fit line is now much more similar. Figure 3.12 (b) shows a comparison of the three other systems, which show some variations in slope but all still remain very similar.

(a)

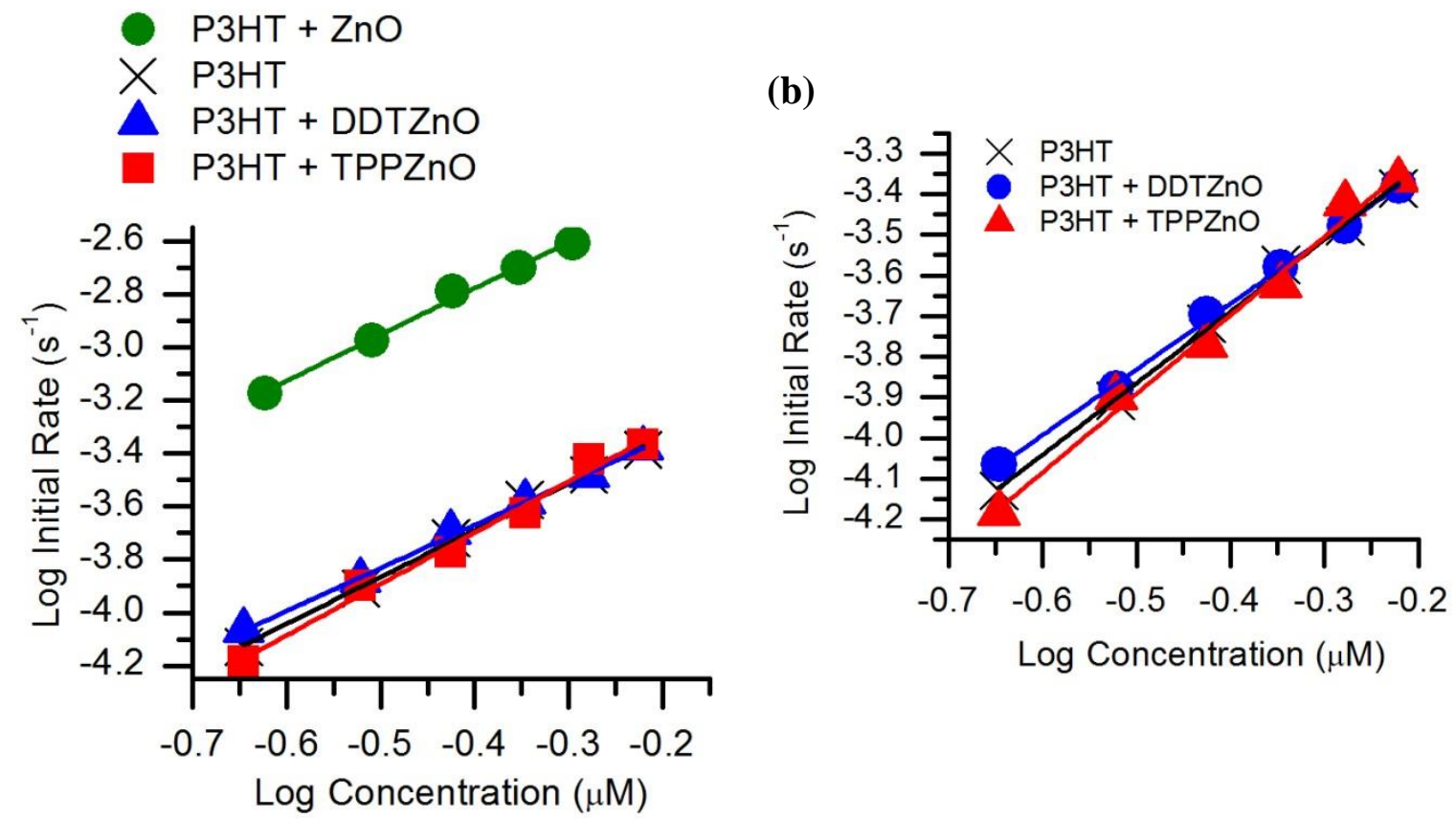

Figure 3.12. Log-log plot of kinetic data for the determination of reaction order of P3HT, P3HT with ZnO, P3HT with DDT-modified ZnO, and P3HT with TPP-modified ZnO.

All four systems are shown in (a), while the P3HT with $\mathrm{ZnO}$ kinetics (done at a different $\mathrm{T}_{\mathrm{c}}$ ) were removed in (b) for clarity.

The results of Figures 3.11 and 3.12 are displayed in Table 3.1. The reaction constant for $\mathrm{P} 3 \mathrm{HT}$ with $\mathrm{ZnO}$ is understandably much higher than the other three due to the lower $\mathrm{T}_{\mathrm{c}}$ during the experiment. However, no obvious trend is seen in the average 
reaction order of these four systems. Repeat experiments produce similar results for all four systems and the reaction order appears to be around 1.6 for all systems.

Table 3.1. Experimentally determined rate constants and reaction orders.

\begin{tabular}{ccc}
\hline System & Rate Constant $\left(\mathbf{s}^{-\mathbf{1}}\right)$ & Average Reaction Order \\
\hline P3HT & $0.0154\left(\mathbf{3 5}^{\circ} \mathbf{C}\right)$ & $1.5 \pm 0.3$ \\
P3HT/ZnO & $0.1076\left(\mathbf{2 5}^{\circ} \mathbf{C}\right)$ & $1.6 \pm 0.1$ \\
P3HT/DDT-ZnO & $0.0136\left(\mathbf{3 5}^{\circ} \mathbf{C}\right)$ & $1.5 \pm 0.2$ \\
P3HT/TPP-ZnO & $0.0172\left(\mathbf{3 5}^{\circ} \mathbf{C}\right)$ & $1.8 \pm 0.1$
\end{tabular}

This is a curious result that suggests two important principles. The first is that because all four systems display approximately the same reaction order it is likely the crystallization mechanism is unchanged when unmodified or modified nanowire is added to the system. This is yet more evidence of non-interaction of the nanowire with polymer or possibly poor surface modification of the nanowire itself is preventing the two materials from interacting with each other in dilute solution. The second principle that this results suggests is that the crystallization mechanism of $\mathrm{P} 3 \mathrm{HT}$ in solution is noninteger. Typical reactions incorporate unimolecular kinetics, or sometimes inverse or bimolecular kinetics. ${ }^{[71]}$ These integer kinetics are due to the interactions with reactants of the same type. For instance, if a reactant has no influence on the rate of other reactants, the reaction order will be first order, because an increase in concentration will simply increase the rate in a linear fashion. In the example below, the reaction is second order with respect to nitrogen monoxide and first order with respect to hydrogen gas. This is due to the bimolecular nature of the reaction, in which two nitrogen monoxide molecules are required to align to form product.

$$
2 \mathrm{NO}(\mathrm{g})+2 \mathrm{H}_{2}(\mathrm{~g}) \longrightarrow \mathrm{N}_{2}(\mathrm{~g})+2 \mathrm{H}_{2} \mathrm{O}(\mathrm{g})
$$


This same principle can be applied to crystallization kinetics, except in the case of P3HT multiple processes are occurring at once that combine to produce P3HT crystals. Referring back to Figure 1.15, after coil-like polymer has transitioned into rod-like polymer it then prefers to crystallize on itself through chain folding and form aggregates with neighboring rod-like chains. The process of single chain folding can be viewed as a unimolecular process in which the conformation of other chains in solution will not affect the rate of folding of a single chain with itself. On the other hand, the aggregation of multiple polymer chains can be viewed as a bimolecular process in which a single rodlike polymer chain is required to align with another chain in solution before it can form an aggregate structure. From this model it is potentially possible for the overall rate of crystallization to then be a combination of these two rates and is thus somewhere between first and second order. While this is just a theory, it does provide a potential basis behind why P3HT crystallization behaves in a non-integer fashion. It is also important to note that the rates of single chain folding and the formation of aggregates can also be affected by the amount of neighboring coil-like or rod-like polymer. If, for example, the tendency for polymer chains to aggregate was negatively affected by coil-like polymer because of the larger free volume, then as more coil-like polymer transitioned to rod-like polymer, aggregate formation would increase at an increasing rate. Thus the conformational transitions, crystallization behavior, and aggregate formation are all intertwined effects in a truly complex and dynamic crystallization process. 


\subsubsection{Avrami Analysis}

Similar to the reaction rate law analysis, Avrami analysis also provides information on the polymer crystallization mechanism. Specifically, the kinetics growth rate constant $\mathrm{n}$ provides information about the dimensionality of polymer crystallization. Many polymers exhibit a wide range of this Avrami constant from 2-4. ${ }^{[59-62]}$ The kinetics data required for Avrami analysis should ideally be acquired until a plateau of polymer crystallization occurs. Not allowing the isothermal crystallization to complete could skew the Avrami exponent, but trends in the kinetic growth rate constant should remain. This is the case in Figure 3.13, as the data was originally acquired for use in the method of initial rates, so the crystallization was not allowed to complete.

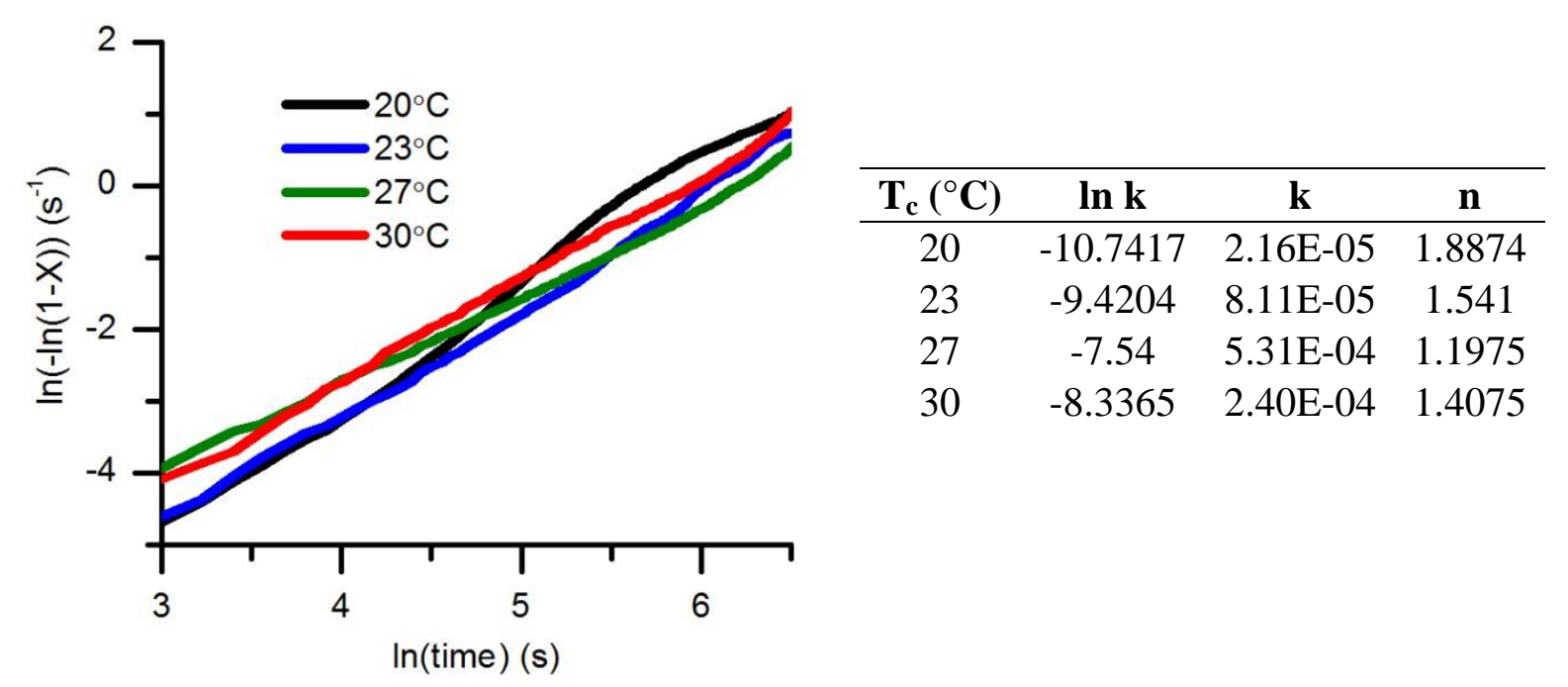

Figure 3.13. Avrami analysis of P3HT crystallization via UV-visible spectroscopy displaying the temperature dependence of the Avrami constants $\mathrm{k}$ and $\mathrm{n}$.

The Avrami analysis for pure P3HT provides some interesting results. First, the kinetic growth rate constant seems to increase with an increase in temperature. This does not agree with theory or most literature results, as an increase in temperature would naturally decrease the tendency for polymers to crystallize via solvent induced 
precipitation. The Avrami exponent also displays a broad range from 1-2, which is lower than expected, but still reasonable. The same type of analysis was then carried out with unmodified and modified $\mathrm{ZnO}$ with P3HT in Figure 3.14. The data obtained for this analysis was originally intended for use in a method of initial rates for LauritzenHoffman kinetics, and is thus on a much shorter time scale than what is ideal.

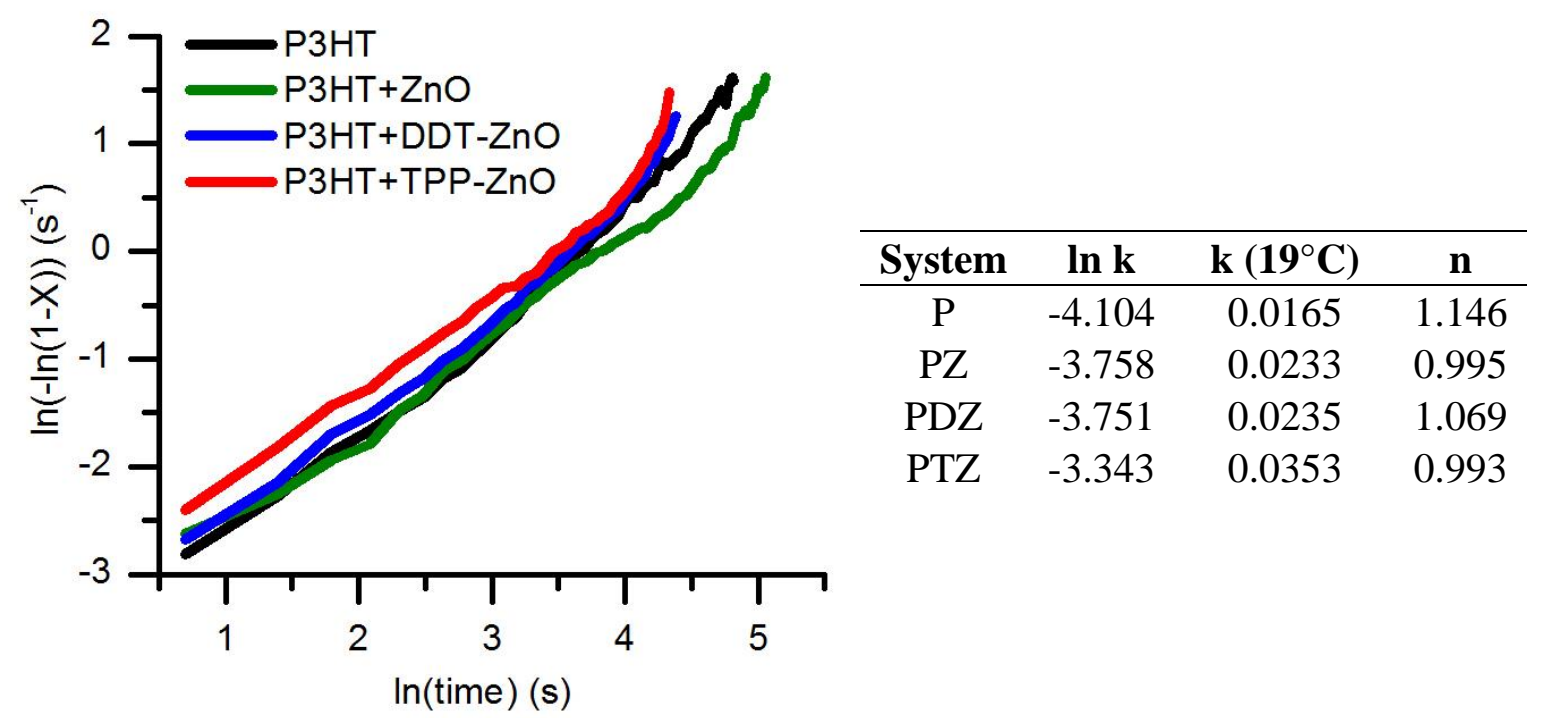

Figure 3.14. Avrami analysis of P3HT, P3HT with ZnO, P3HT with DDT-ZnO and P3HT with TPP-ZnO on a short time scale.

The Avrami exponent for the data remains around 1 for all samples. This contrast from the Avrami exponent determined in Figure 3.13 is likely due to the much shorter time scale seen in Figure 3.14. These initial results also seem to suggest a trend in the kinetic growth rate constant $\mathrm{k}$ with addition of modified $\mathrm{ZnO}$. It is possible that the addition of $\mathrm{ZnO}$ affects the shape of the growth of the polymer crystal, which would reflect in a change in this parameter. However, more precise experimental data designed to fit this analytical method is required to provide any conclusive evidence of this theory.

While Avrami analysis is used frequently to describe polymer crystallization behavior because of its ease of data acquisition, it has many assumptions that are 
frequently overlooked. A few of these assumptions include the constant volume of polymers, constancy in the shape of growing polymer crystals, and complete crystallinity of the sample. While these assumptions may not be met in our experimental analysis, the analysis gives some general idea of the crystallization behavior, and future analysis could yield more precise values.

\subsubsection{Lauritzen-Hoffman Kinetics}

The reaction rate kinetics analysis of these four systems reveals information about the similarity in crystallization mechanism in pure $\mathrm{P} 3 \mathrm{HT}$ and $\mathrm{P} 3 \mathrm{HT}$ with $\mathrm{ZnO}$. But there is a potential for these nanowires to allow P3HT to crystallize more easily. This idea stems from the concept of stem deposition and nucleation sites. Segments of rod-like polymer must first attach somewhere to begin crystallization. This is where modified nanoparticles could potentially provide an advantage. The nanoparticles could create a lower surface energy nucleation site, allowing polymers to begin crystallizing on an already aligned low surface energy rod. To analyze this potential, Lauritzen-Hoffman Growth Theory was employed in-situ with P3HT. To begin, the equilibrium dissolution temperature $\mathrm{T}_{\mathrm{d}}{ }^{0}$ must be acquired for the system. The $\mathrm{T}_{\mathrm{d}}{ }^{0}$ is the temperature at which crystals are energetically as favorable to dissolve as they are to maintain their crystal structure, and is dependent on the polymer-solvent system. Several methods exist to determine $\mathrm{T}_{\mathrm{d}}{ }^{0}$, but perhaps the most widely accepted is the Organ and Keller method, which requires several steps. ${ }^{[67,72-74]}$ The first step requires measuring the $T_{d}$ for a series of crystal solutions that were crystallized at a constant low temperature $\mathrm{T}_{\mathrm{c}}$. This can be accomplished by creating a series of heating curves as seen in Figure 3.15 (a), and the 
finding the inflection point along the curve. The second step is to plot this data alongside a $T_{d}=T_{c}$ line (Figure $\left.3.15(b)\right)$. The intersection point of these lines will reveal the $T_{d}{ }^{0}$ for the polymer-solvent system. This method is the most convenient and easily applicable, but has limitations in terms of accuracy. Choosing crystallization temperatures too low will produce heavily aggregated polymer chains, and the heating curves will be difficult to analyze. Crystallization temperatures too high will take much too long to crystallize and eventually the temperature will not be low enough to allow polymer crystals to form. These effects create a narrow window in which these experiments can be carried out, and thus small errors in measurement will create large differences in the extrapolated best fit line. It is generally accepted that this method is best used to give a general estimate of the $\mathrm{T}_{\mathrm{d}}{ }^{0}$ and the method tends to yield lower values than other methods. ${ }^{[67]}$
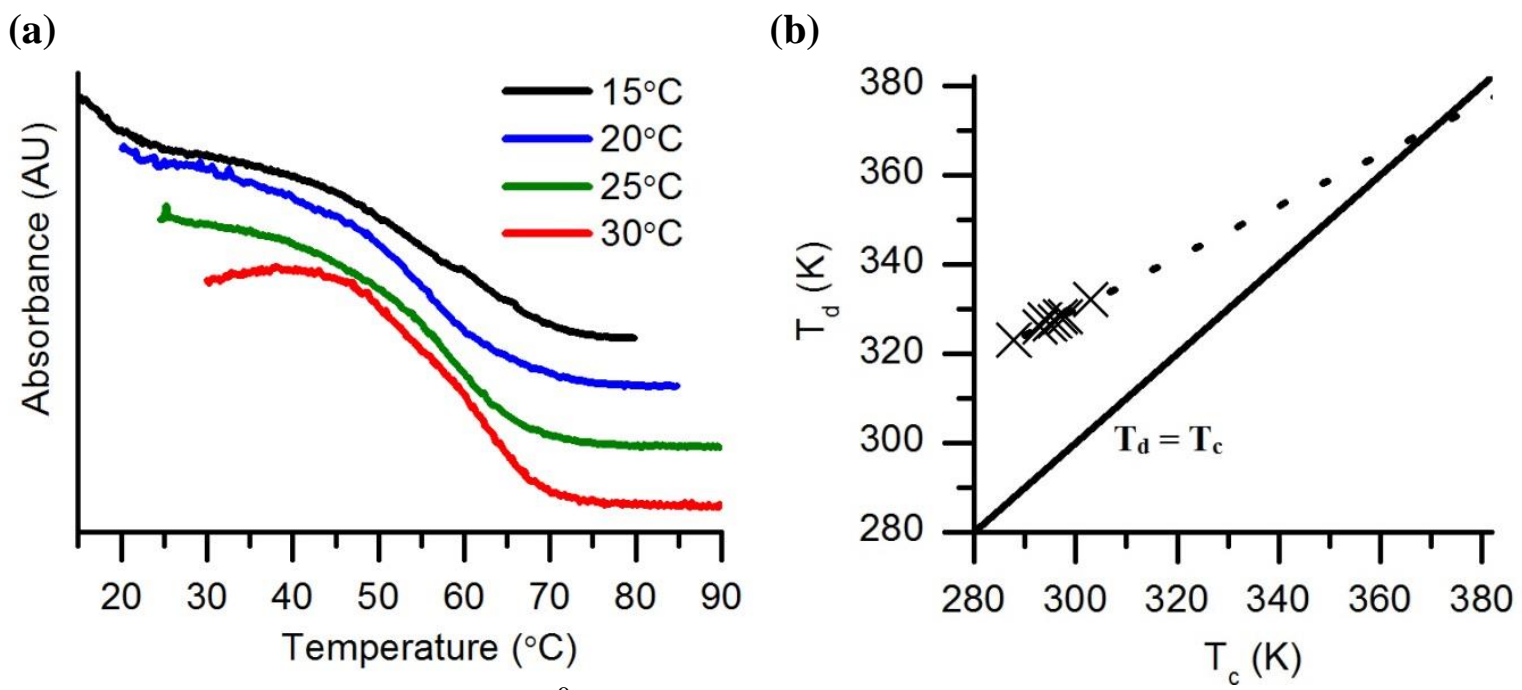

Figure 3.15. Determination of $\mathrm{T}_{\mathrm{d}}{ }^{0}$ for P3HT in anisole using the Organ and Keller method. The heating curves started at different $T_{c}$ 's shown in (a) were plotted against their estimated $\mathrm{T}_{\mathrm{d}}$ values in (b). 
The value of $\mathrm{T}_{\mathrm{d}}{ }^{0}$ was determined to be $371 \mathrm{~K}$. Once the $\mathrm{T}_{\mathrm{d}}{ }^{0}$ has been established, the kinetics of polymer crystallization can be analyzed across varying $\mathrm{T}_{\mathrm{c}}$ 's. The initial rate of these kinetics curves can be estimated through best fit lines, and the data can be plotted as the $\ln \left(\right.$ initial rate) against $1 /\left[\mathrm{T}_{\mathrm{c}}\left(\mathrm{T}_{\mathrm{d}}{ }^{0}-\mathrm{T}_{\mathrm{c}}\right)\right]$ to produce Figure 3.15 . The best fit lines of this plot reveals the $\mathrm{K}_{\mathrm{g}(\mathrm{i})}$ of the system, which can then be converted into surface energy parameters that reveal information about the energetics of $\mathrm{P} 3 \mathrm{HT}$ crystallization. Equations 1.27 and 1.23 are reproduced for convenience.

$$
\begin{gathered}
\ln \left(G_{I}\right)=\left[\ln G_{0}-\frac{U^{*}}{R\left(T_{c}-T_{\infty}\right)}\right]-\frac{K_{g(i)}}{T_{c} \Delta T} \\
K_{g(i)}=\frac{4 b \sigma \sigma_{e} T_{m}^{0}}{k \Delta H_{f}}
\end{gathered}
$$

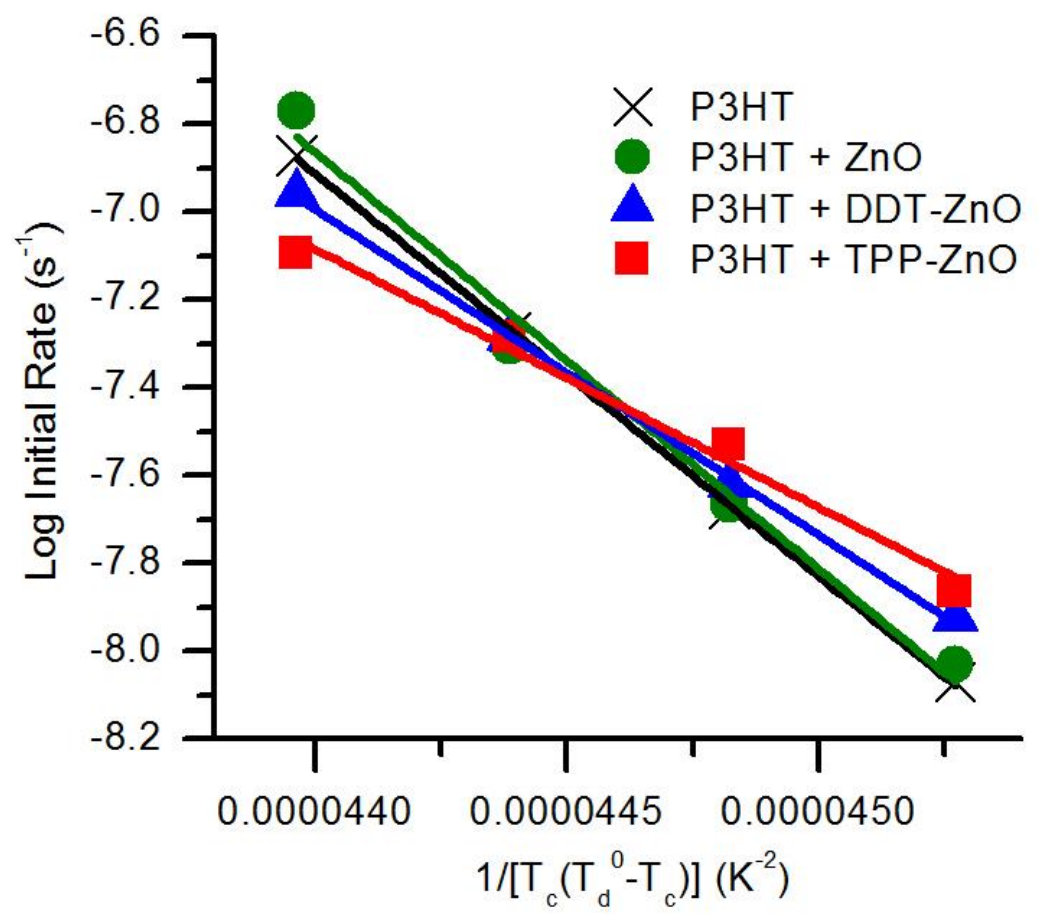

Figure 3.16. Determination of $\mathrm{K}_{\mathrm{g}(1)}$ via L-H Theory for P3HT, P3HT with ZnO, P3HT with DDT-modified $\mathrm{ZnO}$, and P3HT with TPP-modified $\mathrm{ZnO}$. 
Table 3.2 displays the results from Figure 3.16 as well as calculated surface energy parameters for all four systems. Research by Malik et al. with the melt crystallization of poly(alkyl thiophene)s has yielded the following parameters used in the calculation of $\sigma_{\mathrm{e}}$ from Equation $1.23: \mathrm{b}=7.75 \times 10^{-10} \mathrm{~m}, \sigma=1.24 \times 10^{-2} \mathrm{~J} \mathrm{~m}^{-2}$, and $\Delta \mathrm{H}_{\mathrm{f}}=$ $1.096 \times 10^{8} \mathrm{~J} \mathrm{~m}^{-3} \cdot{ }^{[75]}$

Table 3.2. Experimental $\mathrm{K}_{\mathrm{g}}, \sigma \cdot \sigma_{\mathrm{e}}$, and $\sigma_{\mathrm{e}}$ values.

\begin{tabular}{cccc}
\hline System & $\begin{array}{c}\boldsymbol{K}_{\boldsymbol{g}} \\
{\left[\mathbf{K}^{\mathbf{2}}\right] \mathbf{x} \mathbf{1 0}}\end{array}$ & $\begin{array}{c}\boldsymbol{\sigma}^{\mathbf{5}} \boldsymbol{\sigma}_{\boldsymbol{e}} \\
{\left[\mathbf{J}^{\mathbf{2}} \mathbf{~ m}^{\mathbf{4}}\right] \mathbf{x} \mathbf{1 0}}\end{array}$ & $\begin{array}{c}\boldsymbol{\sigma}_{\boldsymbol{e}} \\
{\left[\mathbf{J}_{\mathbf{~ m}}^{\mathbf{- 3}}\right]}\end{array}$ \\
\hline P3HT & 9.16 & 1.28 & 0.103 \\
P3HT + ZnO & 9.40 & 1.31 & 0.106 \\
P3HT + DDT-ZnO & 7.41 & 1.03 & 0.083 \\
P3HT + TPP-ZnO & 5.87 & 0.82 & 0.066
\end{tabular}

The results from Table 3.2 display a trend that is a bit more obvious than the reaction rate analysis. The $\sigma_{\mathrm{e}}$ values are the surface fold energy of the P3HT chain and signify how preferential it is for polymer to crystallize. In general, a lower $\sigma_{\mathrm{e}}$ value allows polymer to crystallize more easily. It would appear from the trend in $\sigma_{\mathrm{e}}$ that unmodified $\mathrm{ZnO}$ decreases polymer crystal formation while DDT- and TPP-modified $\mathrm{ZnO}$ aide in polymer crystal formation. This data does support what is expected, as the modified $\mathrm{ZnO}$ should provide a nucleation site that would allow polymers to crystallize earlier than they would without them. This trend could also be the result of improved miscibility as the modified nanowires are added to solution. The data suggests that the addition of DDT-ZnO nanowire lowers the polymer surface fold energy by $21.5 \%$, while TPP-ZnO nanowire corresponds to a $43.8 \%$ decrease. These values are however not statistically significant, and more kinetics runs must be employed to ensure that these values do in fact show a trend. These decreases in fold energy are quite large in 
comparison to the relative surface area of the modified nanowire. Carbon nanotubes have shown significant decreases in P3HT surface fold energy, and these nanostructures have a much larger surface area per volume than nanowire providing a much larger area for nucleation. ${ }^{[76]}$

\subsection{Quantum Dot Modified Nanowire}

\subsubsection{Quantum Dot Characterization}

The second part of this work is focused on the modification of $\mathrm{ZnO}$ nanowire and subsequent attachment of quantum dots. Figure 3.17 displays characterization of the CdSe quantum dots. The quantum dots are known to absorb light in the visible region, and the UV-visible spectrum reflects this strong absorbance. The spectrum is also weakly dependent on temperature, but this is not substantial enough to affect the band gap of the material across small changes in temperature. The fluorescence spectrum of the quantum dots at an excitation wavelength of $490 \mathrm{~nm}$ displays a sharp peak around $570 \mathrm{~nm}$, in contrast with the P3HT broad emission peak around $580 \mathrm{~nm}$. The fluorescence spectrum increases in intensity with increasing concentration of quantum dots as expected. 
(a)

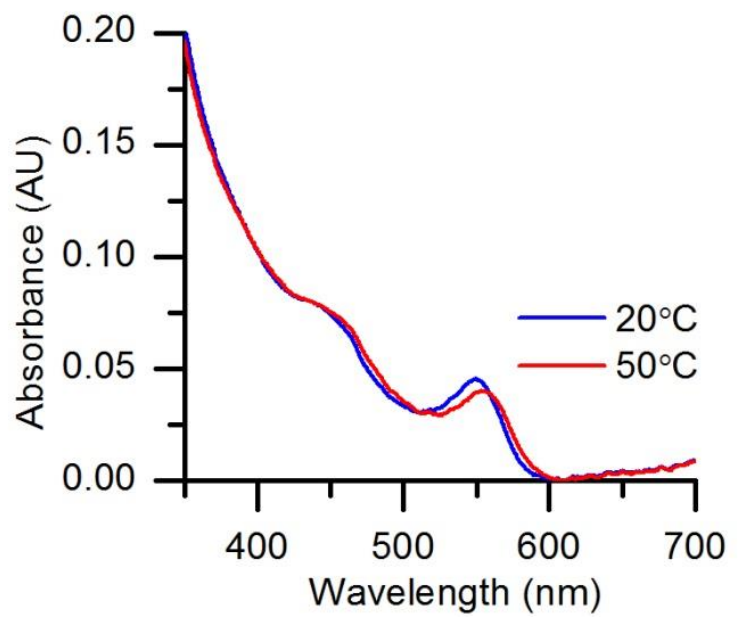

(b)

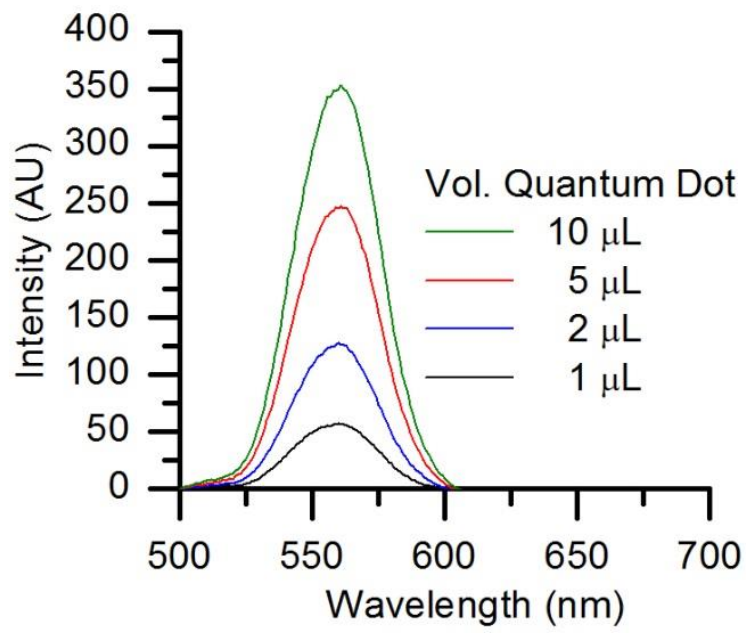

Figure 3.17. Characterization data for CdSe quantum dots. The temperature dependence of the UV-visible absorbance spectrum is explored in (a) and the concentration dependence on the fluorescence spectrum is displayed in (b).

\subsubsection{Effect of Quantum Dots on P3HT Fluorescence}

Before attempting to modify the quantum dots onto $\mathrm{ZnO}$ nanowire, the quantum dots were added by themselves into a polymer solution. If the quantum dots are in close enough proximity to the polymer chains without modification then the fluorescence spectrum of P3HT should exhibit quenching behavior as electrons inject into the quantum dots or vice versa. From Figure 3.18 it is apparent that addition of quantum dots into a solution of P3HT does not affect either spectrum. The spectra of polymer and quantum dot is simply the combined spectra of the two species individually, and provides evidence of non-interaction between the two as expected. 


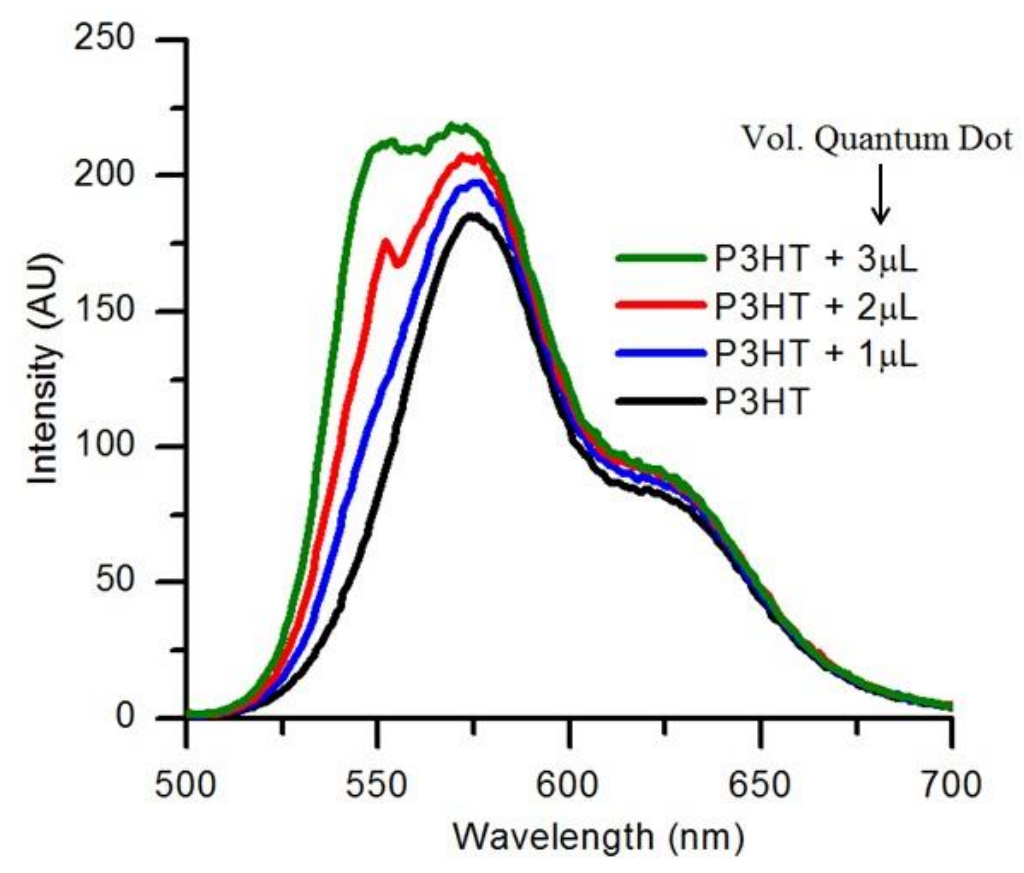

Figure 3.18. The effect of addition of quantum dots on the fluorescence spectrum of P3HT. No quenching is observed, and the spectra resemble the individual P3HT and quantum dot spectra, implying there is little to no interaction as expected.

\subsubsection{Quantum Dot Attachment to Ligand}

The synthetic procedure and concept for this synthetic process was adopted from Zeng et al ${ }^{[39]}$ First, $\mathrm{ZnO}$ nanowire was modified with a bifunctional ligand, with the type of linkage being dependent on the ligand. In the case of 3-Aminopropyltrimethoxysilane (APS), the trimethoxysilane functionality would attach to the $\mathrm{ZnO}$ nanowire surface. In the case of 3-Mercaptopropyltrimethoxysilane (MPS), either the thiol or the trimethoxysilane functionality would provide the link, or more likely a combination of the two. The surface modified $\mathrm{ZnO}$ would then be treated with CdSe quantum dots, which would attach to the amine functionality in the case of the APS ligand or the thiol functionality in the case of the MPS ligand. Zeng et al. developed this procedure to 
modify $\mathrm{ZnO}$ nanocolumns that were already grown on an Indium Tin Oxide (ITO) substrate. Figures 3.19 and 3.20 provide the IR spectra for the species involved. After analysis of these spectra it was determined that the final modified product contained no characteristic features from the quantum dots.

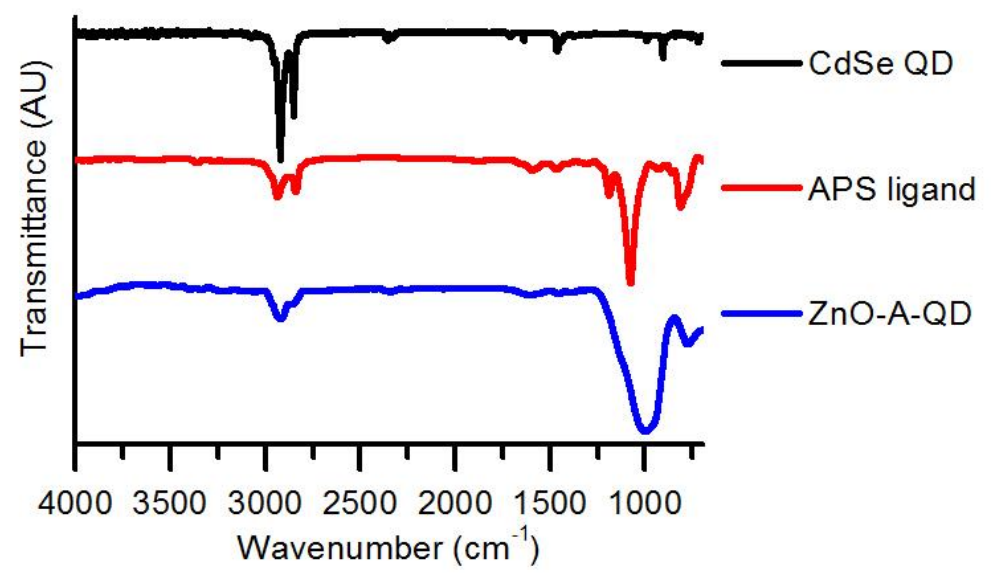

Figure 3.19. IR spectroscopy characterization data for CdSe quantum dots, APS ligand, and APS-modified $\mathrm{ZnO}$ attached to quantum dots.

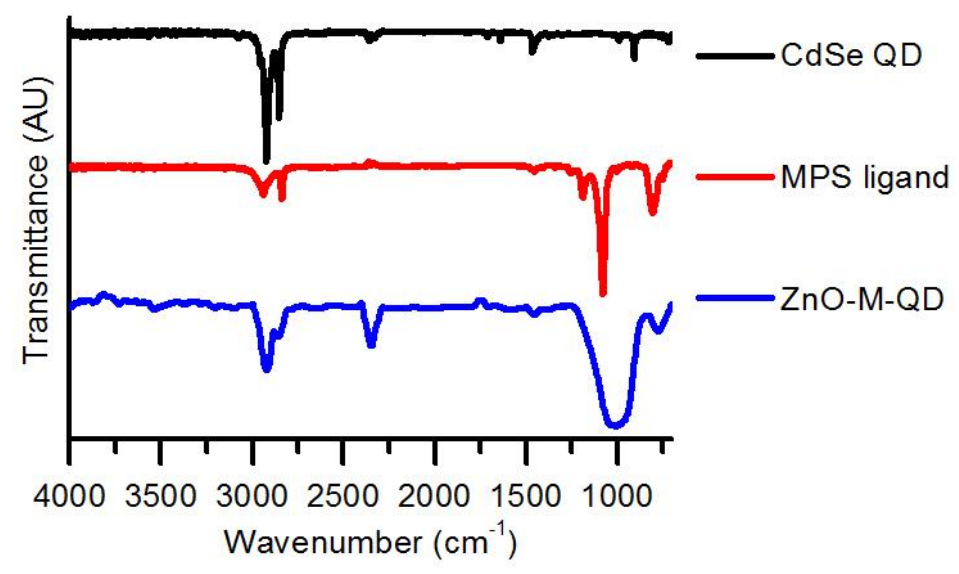

Figure 3.20. IR spectroscopy characterization data for CdSe quantum dots, MPS ligand, and MPS-modified $\mathrm{ZnO}$ attached to quantum dots. 
The first step of the procedure was successful, and the spectra show features of the ligand in both cases. However, after the addition of quantum dots, the solid materials showed no change in color. The CdSe quantum dots used display a very strong orange color, and after vortexing with modified $\mathrm{ZnO}$, the rinse employed to rinse free quantum dots appeared to rinse away all quantum dots. UV-visible spectra of the ZnO-A-QD and $\mathrm{ZnO}-\mathrm{M}-\mathrm{QD}$ revealed no features except that of $\mathrm{ZnO}$, confirming the lack of quantum dots in the final product. It is possible that the adopted procedure does not function in the same manner as the procedure that uses grown nanocolumns of $\mathrm{ZnO}$. Further solvent tests and modification attempts proved unsuccessful, as the ligand could easily attach to the $\mathrm{ZnO}$, but the quantum dots remained solvated. Other literature suggests to chemically bind the ligand to the quantum dot first through slightly different synthetic procedure, but this synthetic approach again provided no attached quantum dots. ${ }^{[41-42]}$ 


\subsection{Research Outlook}

The modified $\mathrm{ZnO}$ systems explored in this work can be directly employed in a hybrid device and tested. The Zhang research group is currently investigating the effects that modifying $\mathrm{ZnO}$ nanowire has on device performance in terms of solar cells. ${ }^{[77]}$ If these devices yield results, the effect of ligand type and size can be explored in much more detail. Different modification techniques could potentially be employed to ensure full surface coverage of the nanoparticles.

One area of very promising research is that of carbon nanotubes and P3HT. The Zhang research group is also working on combining carbon nanotubes and polymer into a BHJ device, where the carbon nanotubes can act as a nucleation site for polymers to crystallize. Preliminary work on this topic has produced positive results, confirming a trend that single-walled carbon nanotubes do indeed create positive interaction with the P3HT side chains via their fully carbon structure. ${ }^{[76]}$

It would also be worthwhile to attempt to investigate the modification procedures through microscopy and other spectroscopic techniques. Raman spectroscopy, for instance, can shed some light on the bonding mode of the TPP ligand. The Raman spectrum for $\mathrm{ZnO}$ displays prominent peaks around 332, 376, 438, and $578 \mathrm{~cm}^{-1}$, indicating its wurtzite structure. These characteristic peaks will shift due to perturbation of the porphyrin center of the ligand if it has bound to $\mathrm{Zn}$ from the $\mathrm{ZnO}$ surface. ${ }^{[68]}$ Fluorescence anisotropy is another technique that could prove useful for confirming the attachment of the TPP ligand. This technique measures the time scale of the rotation of the prophyrin center as it spins. The attachment of the ligand to a bulky nanowire would 
display a decrease in the time scale of this spinning and confirm the surface modification. $^{[78]}$

The CdSe quantum dot coated nanowire is a promising project, but has many synthetic challenges associated with it. Discovering an appropriate solvent system and reaction parameters that will allow the quantum dot to attach to a functional group would open up many possibilities. The difficulty in this becomes understanding the outer layer of the quantum dot, because the CdSe quantum dots are coated with a trioctylphosphineoxide (TOPO) ligand. Thus, attachment to nanowire really requires knowledge of ligand exchange chemistry and might require the use of acid or base.

If these modified $\mathrm{ZnO}-\mathrm{CdSe}$ nanostructures could be synthesized, L-H theory can be employed to directly measure any effects on crystallization. These nanostructures can also be employed in hybrid devices, and the effect of ligand type and size can be explored. Fluorescence spectroscopy can also be employed to measure any electron transfer. And lastly, fluorescence micrographs (Figure 3.21) can be taken of the nanoparticles on the polymer surface to provide information on the directionality of charge transport. ${ }^{[40]}$ 

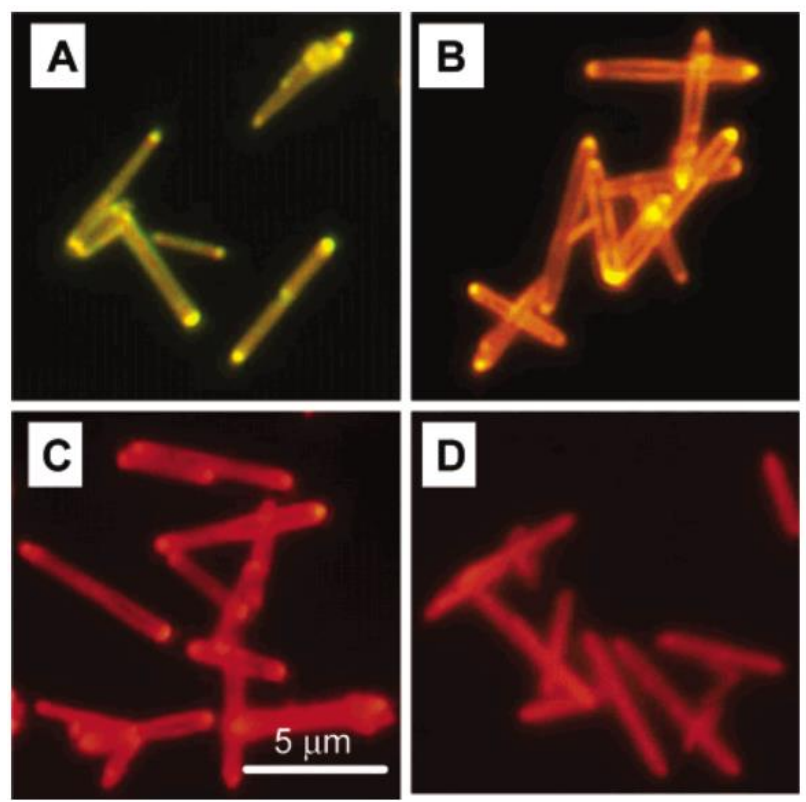

Figure 3.21. Fluorescence micrographs of $\mathrm{ZnO}-\mathrm{CdSe}$ clusters on a $\mathrm{Si}$ wafer in air (A-C) and in hydrocarbon oil with $\mathrm{n}=1.59$ (D). ${ }^{[40]}$

One last area of potential investigation is that of the multi-faceted crystallization mechanism of P3HT. As stated before, P3HT crystallization is really a combination of a conformational transition, single chain folding, and aggregation. It may be possible to set up kinetics experiments that could decouple some of the transitions, leaving one of the three processes available to observe alone. This would provide a very challenging project, but could shed some light on areas of polymer crystallization science that have been hidden for quite some time. 


\section{Conclusions}

In summary, $\mathrm{ZnO}$ nanowire was successfully modified with two ligands to produce hydrophobic inorganic nanowire. The modified nanowire seemed to have no electron transfer behavior when added to P3HT solution. The kinetics of P3HT crystallization with and without this nanowire was investigated, and the mechanism for crystallization remained unchanged. L-H theory was employed to analyze the potential of the modified nanowire to act as a nucleation site and lower the surface fold energy of the polymer. Unmodified $\mathrm{ZnO}$ seemed to slightly hinder polymer crystallization, while the data suggests that DDT-modified $\mathrm{ZnO}$ lowers the energy barrier of crystallization by $21.5 \%$ and TPP-modified $\mathrm{ZnO}$ by $43.8 \%$.

Also, two types of ligands were chemically bonded to $\mathrm{ZnO}$ nanowire in hopes that quantum dots could attach to the ends of these ligands. It was found that the quantum dots did not attach to the $\mathrm{ZnO}$ nanowire through the ligand through simple synthetic methods.

This work has been focused on improving interactions between organic and inorganic material for use in hybrid devices. The interface between these materials is crucial in generating charge carriers, yet little is understood. It is critical to be able to control the morphology of the polymer layer and modifying the inorganic layer is one of the simplest and most effective methods to accomplish this. The development of L-H Theory in conjunction with UV-visible spectroscopy to analyze polymer crystallinity lays the ground work for future advancements in the field of hybrid electronic devices. 
5. References

[1] Inzelt, G., Conducting Polymers - A New Era in Electrochemistry. Berlin, 2008.

[2] Ito, T. S., Hideki; Ikeda, Sakuji, Journal of Polymer Science: Polymer Chemistry Edition 1974, 12, 11-20.

[3] Lalama, S. J. G., A.F.; Shirakawa, H; Macdiarmid, A.G., Bulletin of the American Physical Society 1977, 22 (3), 431.

[4] Shirakawa, H. L., E.J.; Macdiarmid, A.G.; Chiang, C.K.; Heeger, A.J., Journal of the Chemical Society - Chemical Communications 1977, (16), 578-580.

[5] Macdiarmid, A. G. A., M.; Chaing, C.K., Journal of the Electrochemical Society 1977, 124 (8), C304.

[6] Chiang, C. K. F., C.R.; Park, Y.W.; Heeger, A.J., Physical Review Letters 1977, 39 (17), 1098-1101.

[7] Kungl. Vetenskapsakademien, T. R. S. A. o. S., The Nobel Prize in Chemistry, 2000: Conductive Polymers. 2000; pp 1-15.

[8] Forrest, S. Energy Efficiency with organic electronics: Ching W. Tang revisits his days at Kodak Cambridge Journals Online MRS Bulletin [Online], 2012.

[9] Cataldo, S. P., Bruno, Materials 2013, 6, 1159-1190.

[10] Katz, H. E. H., Jia, Annual Review of Materials Research 2009, 39, 71-92.

[11] Abdulrazzaq, O. A., Particulate science and technology 2013, 31 (5), 427-442.

[12] Sproul, A., Understanding the p-n Junction. Solar Cells: Resources for the Secondary Science Teacher pp 13-24.

[13] Moule, A. J. C., Lilian; Thambidurai, Chandru; Vidu, Ruxandra, Stroeve, Pieter, Journal of Materials Chemistry 2012, 22, 2351-2368. 
[14] Laboratories, N. R. E., Best Research Cell Efficiencies. Department of Energy: 2013.

[15] Li, F. D., Yanhui; Chen, Yiwang; Chen, Lie; Zhao, Jie; Wang, Peishan, Solar Energy Materials \& Solar Cells 2012, 97, 64-70.

[16] Bhongale, C. J. T., Mukundan, Solar Energy Materials \& Solar Cells 2010, 94, $817-822$.

[17] Worfolk, B. J. R., David A.; Elias, Anastasia L.; Thomas, Michael, Harris, Kenneth D.; Buriak, Jillian M., Advanced Functional Materials 2011, 21, 1816-1826.

[18] Redeker, N. D. D., Cameron D.; Ding, Yong; Zhang, Shanju, Polymer 2013, 54.

[19] Allen, C. G. B., D. J.; Brenner, T. M.; Weigand, C. C.; Albin, J. M.; Steirer, K. X.; Olson, D. C.; Ladam, C.; Ginley, D. S.; Collins, R. T.; Furtak, T. E., Journal of Physical Chemistry C. 2012, 116, 8872-8880.

[20] Park, B. L., Jung-Hyun; Chang, Mincheol; Reichmanis, Elsa, Journal of Physical Chemistry C. 2012, 116, 4252-4258.

[21] Monson, T. C. L., Matthew T.; Olson, Dana C.; Lee, Yun-Ju; Hsu, Julia W. P., Advanced Materials 2008, 20, 4755-4759.

[22] Rakshit, T. M., Suvra P.; Manna, Indranil; Ray, Samit K., Applied Materials \& Interfaces 2012.

[23] Zhang, S. M., Pawel W.; Keskar, Gayatri; Pfefferle, Lisa D.; Osuji, Chinedum O., Langmuir 2011, 27, 11616-11621.

[24] Zhang, B. K., Tao; Xu, Wenzhi; Su, Ruigong; Gao, Yunhua; Cheng, Guosheng, Langmuir 2010, 26 (6), 4514-4522. 
[25] Allen, C. G. B., D. J.; Albin, J. M.; Oertli, H. E.; Gillaspie, D. T.; Olson D. C.;

Furtak, T. E.; Collins, R. T., Langmuir 2008, 24, 13393-13398.

[26] Weickert, J. D., Ricky B.; Hesse, Holger C.; Wiedemann, Wolfgang, Advanced Materials 2011, 23, 1810-1828.

[27] Gao, F. R., Shenquang; Wang, Jianpu, Energy \& Environmental Science 2013, 6, 2020-2040.

[28] Ren, S. C., Liang-Yi; Lim, Sung-Keun; Zhao, Jing; Smith, Matthew; Zhao, Ni;

Bulovic, Vladimir; Bawendi, Moungi; Gradecak, Silvija, Nano Letters 2011, 11, 39984002.

[29] Zhao, L. W., Jun; Lin, Zhiqun, Frontiers of Chemistry in China 2010, 5 (1), 33-

44.

[30] Huynh, W. U. D., Janke, J.; Alivisatos, Paul, Science 2002, 295, 2425-2427.

[31] Medina-Gonzalez, Y. X., William Z; Chen, Bo; Farhanghi, Nasrin; Charpentier, Paul A., Nanotechnology 2011, 22 (065603), 1-8.

[32] Said, A. J. P., G.; Martini, C.; Ferry, D.; Marine, W.; Giorgio, S.; Fages, F.; Hocq, J.; Boucle, J.; Nelson, J.; Durrant, J. R.; Ackermann, J., Journal of Physical Chemistry 2010, 114, 11273-11278.

[33] Ruankham, P. M., Lea; Sagawa, Takashi; Nakazumi, Hiroyuki; Yoshikawa, Susumu, Journal of Physical Chemistry C. 2011, 115, 23809-23816.

[34] Huang, Y.-C. H., Jui-Hung; Liao, Yu-Chia; Yen, Wei-Che; Li, Shao-Sian; Lin, Shiang-Tai; Chen, Chun-Wei; Su, Wei-Fang, Journal of Materials Chemistry 2011, 21, 4450-4456. 
[35] Liu, D. W., Wei; Qiu, Yongfu; Yang, Shihe; Xiao, Si; Wang, Qu-Quan; Ding, Lu; Wang, Jiannong, Langmuir 2008, 24, 5052-5059.

[36] Zakavi, S. G., Nasrin Gholami, Polyhedron 2007, 26, 2425-2432.

[37] Roales, J. P., Jose M.; Castillero, Pedro; Cano, Manuel; Richardson, Tim H.; Barranco, Angle; Gonzalez-Elipe, Agustin R., Applied Materials \& Interfaces 2012, 4, $5147-5154$

[38] Taratula, O. G., Elena; Wang, Dong; Chu, Dorothy; Zhang, Zheng; Chen, Hanhong; Saraf, Guarav; Lu, Yicheng, Journal of Physical Chemistry B. 2006, 110, 6506-6515.

[39] Zeng, T.-W. L., I-Shuo; Hsu, Fang-Chi; Huang, Kuo-Tung; Liao, Hsuieh-Chung; Su, Wei-Fang, Optics Express 2010, 18 (103), 357-365.

[40] Kim, J. Y. O., Frank E., Journal of the American Chemical Society 2005, 127, $10152-10153$.

[41] Kumar, A. L., Kuan-teh; Madaria, Anuj R.; Zhou, Chongwu, Nano Research 2011, 4 (12), 1181-1190.

[42] Leschkies, Nano Letters 2007, 7 (6), 1793-1798.

[43] Luan, C. V., Aleksander; Susha, Andrei; Xu, Xueqing; Wang, Hong-En; Chen, Xue; Xu, Jun; Zhang, Wenjun; Lee, Chun-Sing; Rogach, Andrey L.; Zapien, Juan Antonio, Nanoscale Research Letters 2011, 6 (340), 1-8.

[44] Salaneck, W. R. F., R. H.; Bredas, J. L., Physics Reports 1999, 319, 231-251.

[45] Ajayaghosh, A., Chemical Society Reviews 2003, 32, 181-191.

[46] Rodd, C. M. R. A., Ritesh, Nano Letters 2011, 11, 3460-3467.

[47] Mandelkern, L., Crystallization of Polymers. McGraw-Hill: New York, 1964. 
[48] Stejny, J. D., J. Keller A., J. Mater. Sci. 1977, 14, 1291.

[49] Ratta, V. Crystallization, Morphology, Thermal Stability and Adhesive Properties of Novel High Performance Semicrystalline Polyimides. Virginia Polytechnic Institute and State University, 1999.

[50] Flory, P. J., Principles of Polymer Chemistry. Cornell University Press: Ithaca, N.Y., 1953.

[51] Herman, K. G., O.; Abitz, W. Z., Phys. Chem. 1930, B10, 371.

[52] Keller, A., Farady Discussions of the Royal Society of Chemistry 1979, n68, 145.

[53] Keller, A., Rep. Prog. Phys. 1968, 31 (623).

[54] Flory, P. J., Structural Orders in Polymers. Permagon Press: New York, 1981.

[55] Mandelkern, L., Characterization of Materials in Research: Ceramics and Polymers. Syracuse Univ. Press: Syracuse, New York, 1975.

[56] Fischer, E. W. S., M.; Dettenmair, M., Farady Discussions of the Royal Society of Chemistry 1979, n68, 263.

[57] Rughooputh, S. D. D. V. H., S.; Heeger, A. J.; Wudl, F., Journal of Polymer Science: Part B: Polymer Physics 1987, 25, 1071-1078.

[58] Atkins, P. P., Julio de, Physical Chemistry 9th edition. 2009.

[59] Kalkar, A. K. D., Vineeta D.; Vatsaraj, Bhakti S., Thermochimica Acta 2013, 568, 74-94.

[60] Gupta, A. C., Veena, J Therm Anal Calorim 2012, 114, 643-651.

[61] Rahaman, H. T., Hideto, Journal of Applied Polymer Science 2013.

[62] Shashidhara, G. M. K. D., S.H.; Preethi, S., Polymer Science Series A 2012, 55

(6), 393-403. 
[63] Hoffman, J. D. M., R. L., Polymer 1997, 38, 3682.

[64] Hoffman, J. D. D., G. T.; Lauritzen, J. I., Treatise on Solid State Chemistry.

Plenum Press: New York, 1976; Vol. 3.

[65] Frank, F. C. T., M., Proc. R. Soc. London, Ser. A. 1961, 263, 323.

[66] Snyder, C. R. M., H., Macromolecules 1997, 30, 2759.

[67] Ding, N. A., Eric J., Macromolecules 1991, 24, 3906-3914.

[68] Sarkar, S. M., Abdhinandan; Bora, Tanujjal; Lakhsman, Karthik; Singha, Achintya; Dutta, Joydeep; Pal, Samir Kumar, Applied Materials \& Interfaces 2012, 4, $7027-7035$.

[69] Liu, J. Z., Jianhua; Zhai, Lei, Macromolecular Rapid Communications 2009, 30, 1387-1391.

[70] Oh, J. Y. S., Minkwan; Lee, Tae Il; Jang, Woo Soon; Min, Yuho; Myoung, JaeMin; Baik, Hong Koo; Jeong, Unyong, Macromolecules 2012, 45, 7504-7513.

[71] Danesh, C. D. S., Nathan S.; Zhang, Shanju, Journal of Physical Chemistry B. 2012, 116, 12887-12894.

[72] Ding, N. A., Eric J., Macromolecules 1991, 24, 6464-6469.

[73] Pal, S. N., Arun K., Polymer 2005, 46, 8321-8330.

[74] Sanchez, I. C. D., Edmund A., Macromolecules 1971, 4 (6), 677-687.

[75] Malik, S. N., Arun K., Journal of Polymer Science: Part B: Polymer Physics 2002, 40, 2073-2085.

[76] Luo, G., Carbon Nanotubes and Poly(3-hexylthiophene) Crystallization Behavior. California Polytechnic State University, San Luis Obispo: 2013. 
[77] Olson, G., Surface Modified $\mathrm{ZnO}$ and Poly(3-hexylthiophene) Hybrid Electronic Devices. California Polytechnic State University, San Luis Obispo: 2013.

[78] Piszczek, G., Archives of Biochemistry and Biophysics 2006, 453, 54-62. 